\title{
DOTAM-Based, Targeted, Activatable Fluorescent Probes for the Highly Sensitive and Selective Detection of Cancer Cells
}

Benjamin Brennecke ${ }^{1}$, Qinghua Wang ${ }^{1}$, Wolfgang Haap, Uwe Grether, Hai-Yu Hu*, and Marc Nazaré*

${ }^{1}$ these authors contributed equally

\section{Supporting Information}

\section{Corresponding Authors}

Marc Nazaré - Medicinal Chemistry, Leibniz-Forschungsinstitut für Molekulare

Pharmakologie Berlin, 13125 Berlin, Germany; Email: nazare@fmp-berlin.de

Hai-Yu Hu - State Key Laboratory of Bioactive Substances and Function of Natural Medicine, Institute of Materia Medica, Peking Union Medical College and Chinese Academy of Medical Sciences, Beijing 100050, China; Email: haiyu.hu@imm.ac.cn

\footnotetext{
Authors

Benjamin Brennecke - Medicinal Chemistry, Leibniz-Forschungsinstitut für Molekulare Pharmakologie Berlin, 13125 Berlin, Germany.

Qinghua Wang - State Key Laboratory of Bioactive Substances and Function of Natural Medicine, Institute of Materia Medica, Peking Union Medical College and Chinese Academy of Medical Sciences, Beijing 100050; China.

Wolfgang Haap - Roche Innovation Center Basel, Pharma Research and Early Development, F. Hoffmann-La Roche Ltd., 4070 Basel, Switzerland.

Uwe Grether - Roche Innovation Center Basel, Pharma Research and Early Development, F. Hoffmann-La Roche Ltd., 4070 Basel, Switzerland.
} 


\section{Table of contents}

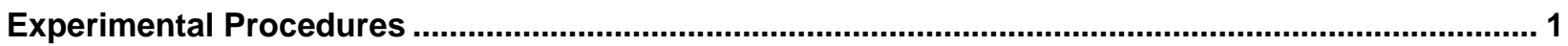

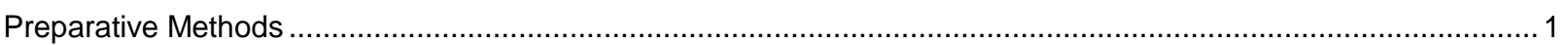

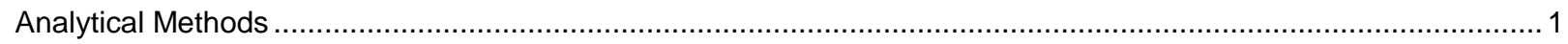

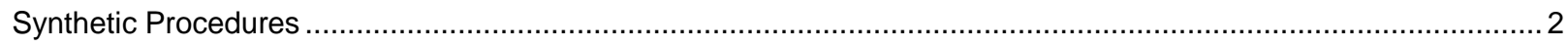

Preparation of cathepsin S sequence S1

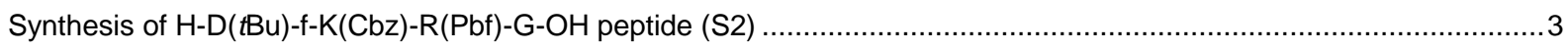

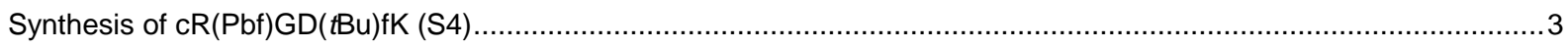

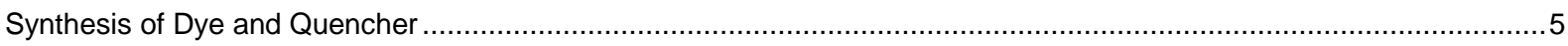

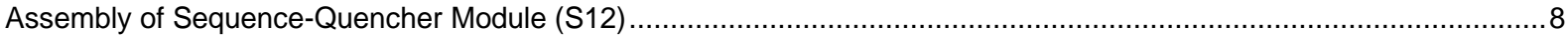

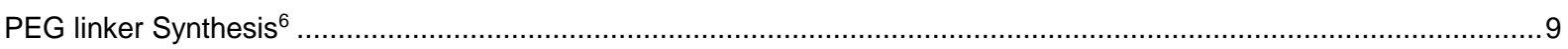

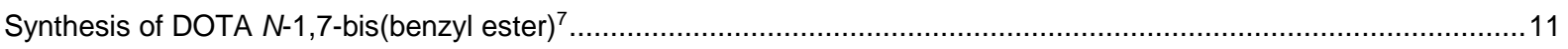

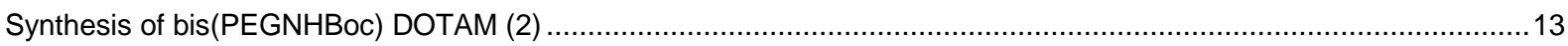

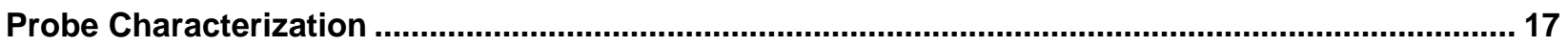

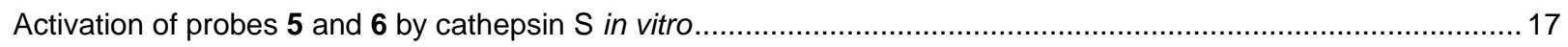

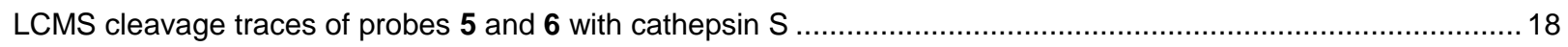

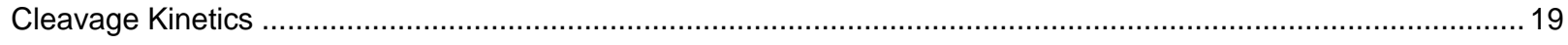

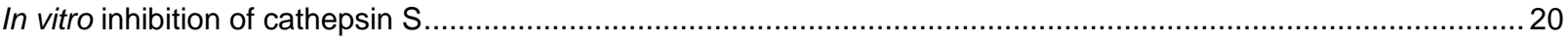

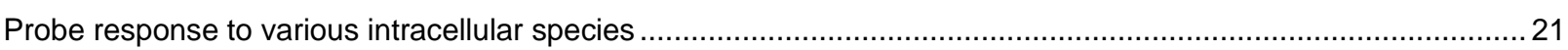

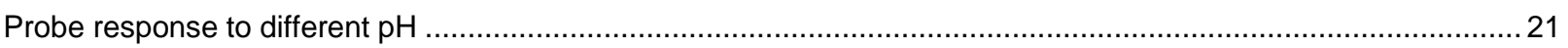

Co-localization experiments of probes 5 and $\mathbf{6}$ with MitoTracker Green in HT1080 cells.................................22

Co-localization experiments of probe 5 with Lyso- and MitoTracker in MCF-7 cells ............................................. 23

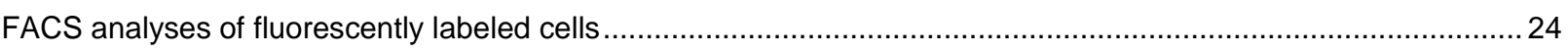

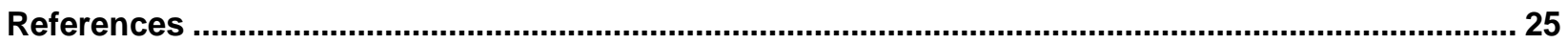

Appendix: NMR spectra and LCMS traces of new compounds and key compounds...................... 25 


\section{Experimental Procedures}

\section{Preparative Methods}

Reactions requiring anhydrous conditions were carried out in dry solvents stored over molecular sieves (THF, DCM, toluene, pyridine, DMF from Sigma Aldrich) which were used as received. These moisture- and air-sensitive reactions were conducted under nitrogen using Schlenk-technique.

Purification of compounds was performed by flash chromatography on silica using a Biotage Isolera One apparatus or by HPLC using a Waters system equipped with a Waters 2489 UV/visible detector, a Waters 2545 Binary Gradient Module and a Nucleodur 100-7 C18ec column from Macherey-Nagel. Water/acetonitrile with $0.1 \%$ TFA was used as the solvent system with the detection wavelength set to $220 \mathrm{~nm}$ or $254 \mathrm{~nm}$.

Fmoc-EDA HCl ((9H-fluoren-9-yl)methyl (2-aminoethyl)carbamate hydrochloride) was synthesized according to the literature. ${ }^{1}$

The unprotected cRGDfK control peptide was prepared according to the literature. ${ }^{2}$

\section{Analytical Methods}

NMR spectra were acquired on devices from the company Bruker (AV 300, AV 600). All ${ }^{13} \mathrm{C}-\mathrm{NMR}$-spectra were recorded with ${ }^{1} \mathrm{H}$-broad-band decoupling. Calibration of the chemical shift was conducted using the solvent residual signals. Numbering of the denoted compounds arises from the IUPAC nomenclature. High resolution mass spectra were recorded with an Agilent 1260 liquid chromatography coupled accurate mass time-of-flight 6230 detector. Standard analysis of reaction time courses was conducted with an Agilent 1260 infinity liquid chromatography coupled quadrupole mass spectrometer 6120 detector equipped with a Thermo Fisher Accucore RP-MS C18 (standard 2.5 min or 8 min method), an Accucore aQ C18 (polar column) and a Knauer Eurosil Bioselect 300-3 C18 (8 min method for biomolecules with molecular weights between 2000 and 5000 Da). 


\section{Synthetic Procedures}

\section{Preparation of cathepsin S sequence S1}
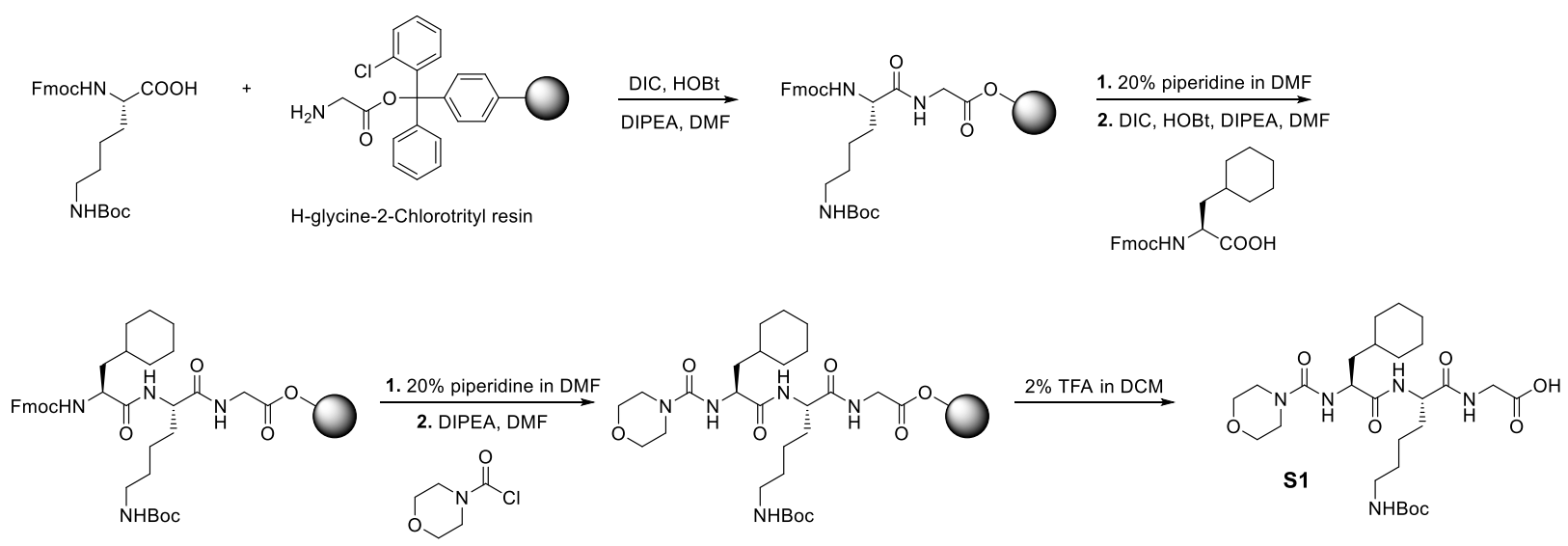

The cathepsin S sequence $\mathbf{S 1}$ was prepared following standard Fmoc strategy solid phase peptide synthesis (SPPS) protocols. The H-glycine-2-chlorotrityl resin (Abbexa, $0.78 \mathrm{mmol} / \mathrm{g}$ loading capacity) was used as solid support. The resin (1 $\mathrm{g}$ ) was thoroughly washed with DMF, THF and DCM prior to coupling to the first amino acid. Coupling of the respective amino acids was conducted with 2 eq. (1.56 mmol) Fmoc-protected amino acid, 2 eq. (271 $\mu \mathrm{l}, 1.56 \mathrm{mmol})$ DIPEA, 2 eq. (239 mg, 1.56 $\mathrm{mmol})$ hydroxybenzotriazole monohydrate and 2 eq. $(242 \mu \mathrm{l}, 1.56 \mathrm{mmol}$ ) diisopropylcarbodiimide in DMF (ca. $3 \mathrm{ml})$. The reaction mixture was shaken at r.t. overnight. After each reaction step the resin was washed successively with DMF, THF and DCM. A small aliquot of the resin was taken after each coupling and subjected to the Kaiser-test or cleaved from the resin with $2 \%$ TFA in DCM and analyzed by LCMS to check if all free amino groups had been reacted. If coupling was not completed, the procedure was repeated with 1-2 eq. Fmoc-protected amino acid. Fmoc deprotection was conducted with $20 \%$ piperidine in DMF (2x15 min). Acylation of the $\mathrm{N}$-terminus was performed using $3 \mathrm{eq}$. 4-morpholinecarbonyl chloride (273 $\mu \mathrm{l}, 2.34 \mathrm{mmol})$ and 3 eq. DIPEA (408 $\mu \mathrm{l}, 2.34 \mathrm{mmol})$ in dry DMF $(3 \mathrm{ml})$. Cleavage of the peptide from the resin was accomplished by treating the resin with $2 \%$ TFA in dichloromethane $(5 \mathrm{ml}, 2 \times 10 \mathrm{~min})$. The cleavage solution was diluted with toluene and the solvents were removed under reduced pressure. The peptide was then redissolved in acetonitrile/water and lyophilized to yield $338 \mathrm{mg}$ $\mathbf{S 1}(76 \%)$ as white powder. The product $\mathbf{S} 1$ was used for the subsequent reaction without further purification. ${ }^{1} \mathbf{H}-\mathbf{N M R}(600$ $\left.\mathrm{MHz}, \mathrm{CDCl}_{3}\right): \delta=0.80-1.91\left(\mathrm{~m}, 28 \mathrm{H}, \mathrm{C}\left(\mathrm{CH}_{3}\right)_{3 \text { Boc }},\left(\mathrm{CH}_{2}\right)_{3 \text { Lys }}, \mathrm{CH}_{2 \text { cHexAla }}\right), 3.04\left(\mathrm{~m}_{\mathrm{c}}, 2 \mathrm{H}, \mathrm{CH}_{2} \mathrm{NH}_{\text {Lys }}\right), 3.28-3.44\left(\mathrm{~m}, 4 \mathrm{H}, \mathrm{N}\left(\mathrm{CH}_{2}\right)_{2}\right)$, $3.66\left(\mathrm{~m}_{\mathrm{c}}, 4 \mathrm{H}, \mathrm{O}\left(\mathrm{CH}_{2}\right)_{2}\right), 3.90-4.12\left(\mathrm{~m}, 2 \mathrm{H}, \mathrm{CH}_{2, \mathrm{Gly}}\right), 4.40\left(\mathrm{~m}_{\mathrm{c}}, 1 \mathrm{H}, \mathrm{CH}_{\mathrm{Lys}}\right), 4.56\left(\mathrm{dt}, \mathrm{J}=5.4,8.5 \mathrm{~Hz}, 1 \mathrm{H}, \mathrm{CH}_{\mathrm{cHexAla}}\right), 5.03,5.61$, 5.69, 7.53, $7.57(\mathrm{~m}, 4 \mathrm{H}, \mathrm{NH})$ ppm. ${ }^{13} \mathrm{C}-\mathrm{NMR}\left(150 \mathrm{MHz}, \mathrm{CDCl}_{3}\right): \delta=22.8,26.2,26.4,26.5,29.5,31.7,32.7,33.8,34.4,39.8$

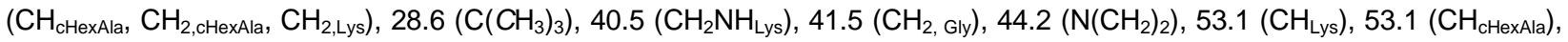
$66.5\left(\mathrm{O}\left(\mathrm{CH}_{2}\right)_{2}\right), 79.5\left(\mathrm{C}\left(\mathrm{CH}_{3}\right)_{3}\right), 156.5\left(\mathrm{NHCO}_{2}\right), 157.9(\mathrm{NCONH}), 172.3\left(\mathrm{CONH}_{\mathrm{Lys}}\right), 172.6\left(\mathrm{CONH}_{\mathrm{cHexAla}}\right), 174.4\left(\mathrm{CO}_{2} \mathrm{H}\right) \mathrm{ppm}$. HRMS (pos. ESI-TOF): $m / z$ calculated for $\mathrm{C}_{27} \mathrm{H}_{48} \mathrm{~N}_{5} \mathrm{O}_{8}[\mathrm{M}+\mathrm{H}]^{+} 570.3497$, found 570.3493 . 
Synthesis of H-D(tBu)-f-K(Cbz)-R(Pbf)-G-OH peptide (S2)

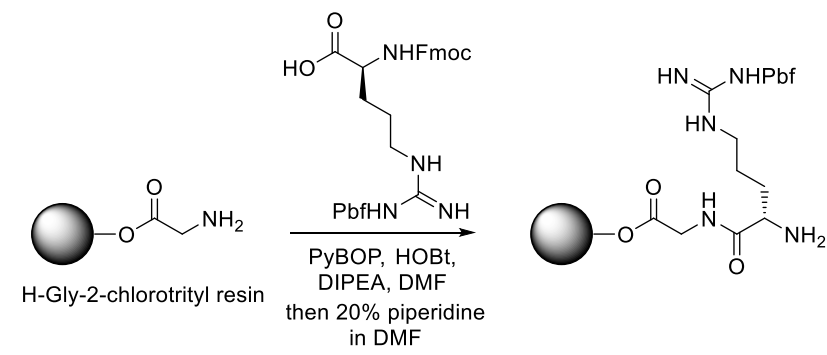

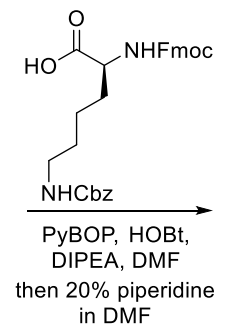
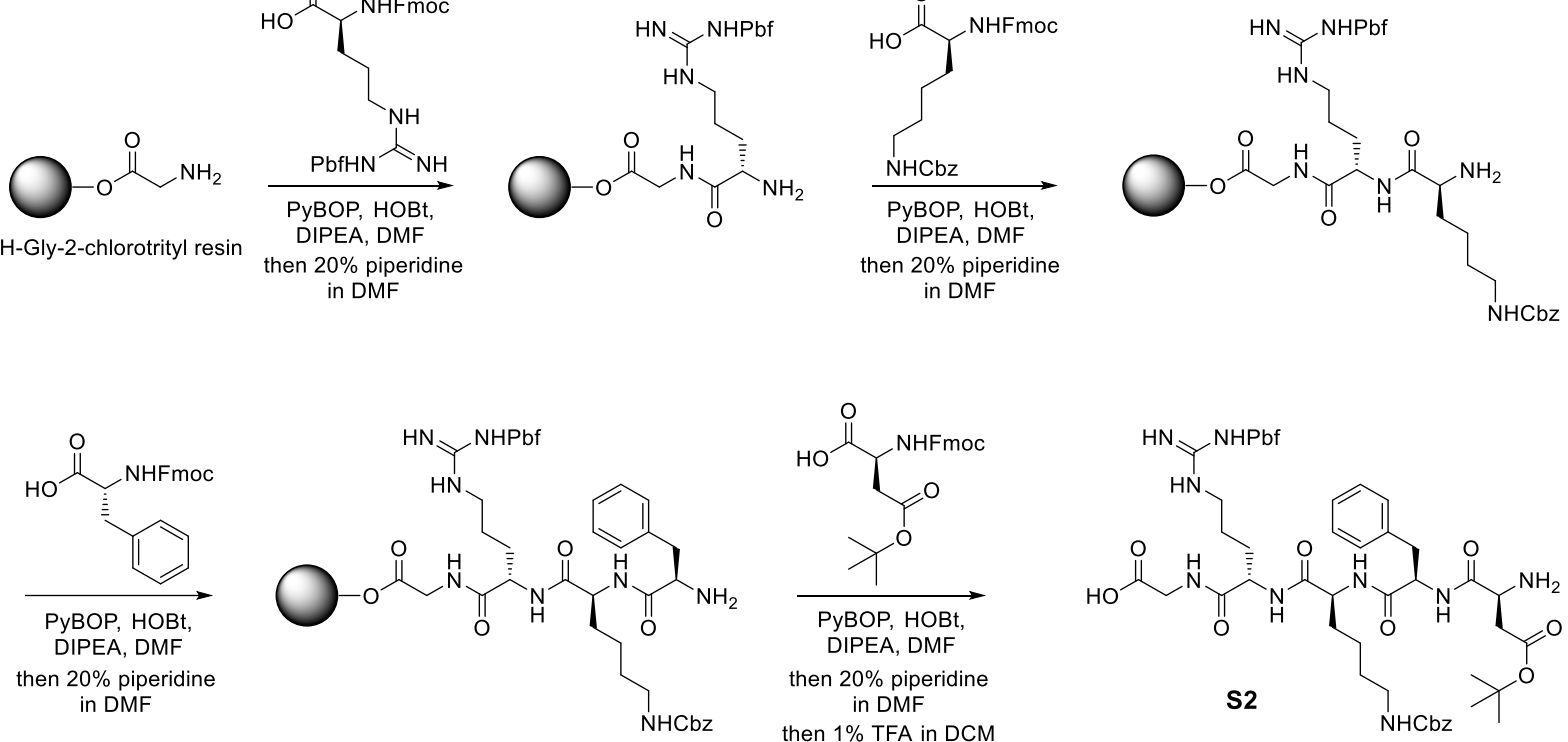

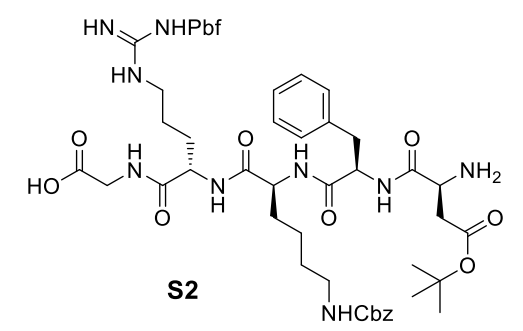

The $\mathrm{R}(\mathrm{Pbf}) \mathrm{GD}\left({ }^{\mathrm{B}} \mathrm{Bu}\right) \mathrm{fK}(\mathrm{Cbz})$ peptide (S2) was prepared following standard Fmoc strategy solid phase peptide synthesis (SPPS) protocols. The H-glycine-2-chlorotrityl resin (Abbexa, $0.87 \mathrm{mmol} / \mathrm{g}$ loading capacity) was used as solid support. The resin $(2 \mathrm{~g}$ ) was thoroughly washed with DMF, THF and DCM prior to coupling to the first amino acid. Coupling of the respective amino acids was conducted with 2 eq. (1.74 mmol) Fmoc-protected amino acid, 3 eq. ( $456 \mu \mathrm{l}, 2.61 \mathrm{mmol})$ DIPEA, 2 eq. (235 mg, 1.74 $\mathrm{mmol}$ ) hydroxybenzotriazole monohydrate and 2 eq. (905 mg, $1.74 \mathrm{mmol}$ ) PyBOP in DMF. The reaction mixture was shaken at r.t. for $2 \mathrm{~h}$. After each reaction step the resin was washed successively with DMF, THF and DCM. A small aliquot of the resin was taken after each coupling and subjected to the Kaiser-test or cleaved from the resin with 1\% TFA in DCM and analyzed by LCMS to check if all free amino groups had been reacted. If coupling was not completed, the procedure was repeated with 1-2 eq. Fmoc-protected amino acid. Fmoc deprotection was conducted with $20 \%$ piperidine in DMF (2x15 min). Cleavage of the peptide from the resin was accomplished by treating the resin with $1 \%$ TFA in dichloromethane $(5 \mathrm{ml}, 5 \times 10 \mathrm{~min})$ and aspirating the cleavage solution into $\mathrm{MeOH} /$ pyridine (9:1). The solvents were removed under reduced pressure and the residue was dissolved in the minimum amount of methanol and precipitated by addition of water. The precipitate was isolated by centrifugation. The product was again dissolved in DCM, washed with water, and dried over $\mathrm{MgSO}_{4}$ to yield $1.06 \mathrm{~g} \mathrm{(57 \% )}$ of the peptide $\mathbf{S} 2$ as colorless resin. ${ }^{1} \mathrm{H}-\mathrm{NMR}\left(600 \mathrm{MHz}, \mathrm{DMF}-\mathrm{D}_{7}\right): \delta=1.18\left(\mathrm{~m}, 2 \mathrm{H}, \mathrm{CHCH}_{2} \mathrm{CH}_{2, \mathrm{Lys}}\right), 1.42\left(\mathrm{~s}, 9 \mathrm{H}, \mathrm{C}\left(\mathrm{CH}_{3}\right)_{3}\right), 1.45$ (s, $\left.6 \mathrm{H}, \mathrm{C}\left(\mathrm{CH}_{3}\right)_{2}\right), 1.40-1.47$ (m, $2 \mathrm{H}, \mathrm{CHCH}_{2} \mathrm{CH}_{2}$, Arg), 1.50-1.65, 1.67-1.83, 1.87-1.95 (4 m, $6 \mathrm{H}, \mathrm{CHCH}_{2, \text { arg }}$, $\mathrm{CHCH}_{2, \text { Lys }}$,

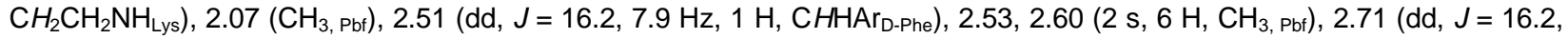
$5.0 \mathrm{~Hz}, 1 \mathrm{H}, \mathrm{CH} H \mathrm{Ar}$-Phe), 3.02 (s, $2 \mathrm{H}, \mathrm{CH}_{2, \text { Pbf }}$ ), 3.02-3.14 (m, $4 \mathrm{H}, \mathrm{CH}_{2} \mathrm{NH}_{\text {Lys }}, \mathrm{CH}_{2}$, Asp), 3.15-3.22 (m, $2 \mathrm{H}, \mathrm{CH}_{2} \mathrm{NH}_{\text {Arg }}$ ), 3.76$3.92\left(\mathrm{~m}, 3 \mathrm{H}, \mathrm{CH}_{2, \text { Gly }}, \alpha-\mathrm{CH}_{\text {D-Phe }}\right.$ ), 4.20 (ddd, $\left.J=9.7,7.4,4.3 \mathrm{~Hz}, 1 \mathrm{H}, \mathrm{a}-\mathrm{CH}_{\mathrm{Lys}}\right), 4.38$ (dt, $J=8.9,4.9 \mathrm{~Hz}, 1 \mathrm{H}, \mathrm{a}-\mathrm{CH}_{\text {Arg }}$ ), 4.63 (m, $\left.1 \mathrm{H}, \alpha-\mathrm{CH}_{\text {Asp }}\right), 5.08\left(\mathrm{~s}, 2 \mathrm{H}, \mathrm{CH}_{2} \mathrm{Ar}_{\mathrm{Lys}}\right), 6.65-7.05(\mathrm{bs}, 3 \mathrm{H}, \mathrm{NH}), 7.11$ (t, $\left.J=5.6 \mathrm{~Hz}, 1 \mathrm{H}, \mathrm{NH}\right), 7.20-7.50\left(\mathrm{~m}, 10 \mathrm{H}, \mathrm{CH}_{\mathrm{Ar}, \mathrm{Pbf}}\right.$, $\left.\mathrm{CH}_{\mathrm{Ar}, \mathrm{Cbz}}\right), 7.84(\mathrm{t}, J=5.6 \mathrm{~Hz}, 1 \mathrm{H}, \mathrm{NH}), 7.95(\mathrm{~d}, J=8.3 \mathrm{~Hz}, 1 \mathrm{H}, \mathrm{NH}), 8.34(\mathrm{~d}, J=7.3 \mathrm{~Hz}, 1 \mathrm{H}, \mathrm{NH}), 8.55(\mathrm{~s}, 1 \mathrm{H}, \mathrm{NH}) \mathrm{ppm} .{ }^{13} \mathrm{C}-$ NMR $\left(150 \mathrm{MHz}, \mathrm{DMF}-\mathrm{D}_{7}\right): \delta=12.9,18.5,19.8,24.1,26.7,26.8,28.6,29.0,39.0,40.9,41.6,42.8,43.8,52.7,53.9,55.0,56.3$, $66.5,81.4,87.4,117.7,125.7,127.6,128.8,129.3,129.5,130.5,133.0,135.9,138.6,138.8,138.9,157.6,157.9,159.0,163.2$, 170.7, 171.6, 171.8, 172.6, 172.9, 174.3. HRMS (pos. ESI-TOF): $\mathrm{m} / z$ calculated for $\mathrm{C}_{52} \mathrm{H}_{74} \mathrm{~N}_{9} \mathrm{O}_{13} \mathrm{~S}[\mathrm{M}+\mathrm{H}]^{+} 1064.5121$, found 1064.5132.

\section{Synthesis of $\mathrm{cR}(\mathrm{Pbf}) \mathrm{GD}(\mathrm{tBu}) \mathrm{fK}(\mathrm{S} 4)$}<smiles>CC(C)(C)OC(=O)C[C@H](N)C(=O)NC(Cc1ccccc1)C(=O)N(CCCCN)C(=O)N[C@@H](CCCNC(=N)NN)C(=O)NCC(=O)O</smiles>

S2

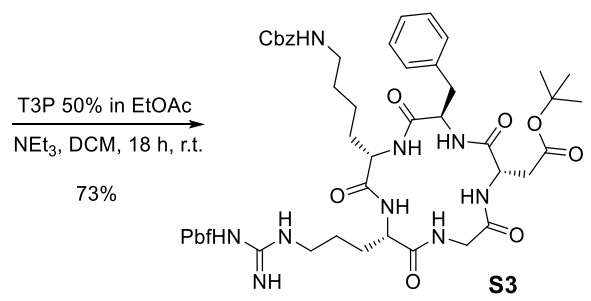

S3

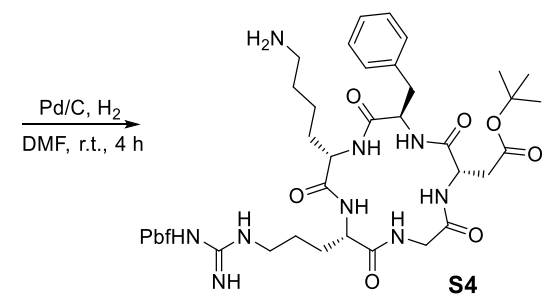




\section{$\mathrm{cR}(\mathrm{Pbf}) \mathrm{GD}(t \mathrm{Bu}) \mathrm{fK}(\mathrm{Cbz})(\mathrm{S} 3)$}

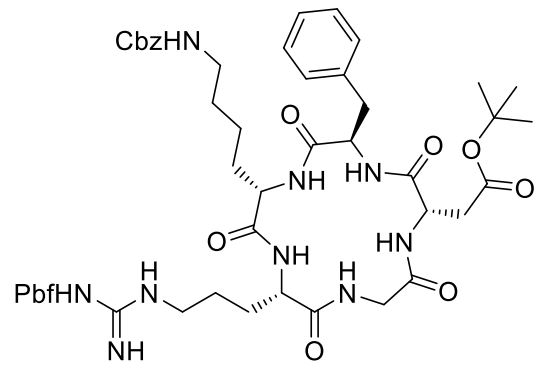

$\mathrm{H}-\mathrm{D}(\mathrm{tBu})-\mathrm{f}-\mathrm{K}(\mathrm{Cbz})-\mathrm{R}(\mathrm{Pbf})-\mathrm{G}-\mathrm{OH}(\mathbf{S} 2,150 \mathrm{mg}, 0.14 \mathrm{mmol})$ was dissolved in DCM $(250 \mathrm{ml})$ together with triethyl amine $(98 \mu \mathrm{l}$, $0.71 \mathrm{mmol})$. This solution was added dropwise during the course of $3 \mathrm{~h}$ to a solution containing T3P $(125 \mu \mathrm{l}, 0.21 \mathrm{mmol})$ in DCM $(100 \mathrm{ml})$ at r.t. The resulting solution was stirred for $18 \mathrm{~h}$ at r.t., washed with $5 \%$ aq. $\mathrm{NaHCO}_{3}$ and water. The organic layer was dried over $\mathrm{MgSO}_{4}$, filtered and the solvents were evaporated under reduced pressure. Purification by flash chromatography $\left(\mathrm{SiO}_{2}\right.$, DCM, $\left.5-10 \% \mathrm{MeOH}\right)$ yielded $114 \mathrm{mg}(77 \%)$ of the title compound (S3) as colorless resin. ${ }^{1} \mathbf{H}-\mathbf{N M R}(600$ MHz, DMF-D $\left.\mathrm{D}_{7}\right): \delta=1.15-1.23\left(\mathrm{~m}, 2 \mathrm{H}, \mathrm{CHCH}_{2} \mathrm{CH}_{2, \mathrm{Lys}}\right), 1.35-1.63\left(2 \mathrm{~m}, 6 \mathrm{H}, \mathrm{CHCH}_{2, \mathrm{Lys}}, \mathrm{CHCH}_{2, \mathrm{Arg}}, \mathrm{CH}_{2} \mathrm{CH}_{2} \mathrm{NH}_{\mathrm{Lys}}\right), 1.39$ (s, 9 $\left.\mathrm{H}, \mathrm{C}\left(\mathrm{CH}_{3}\right)_{3}\right), 1.45\left(\mathrm{~s}, 6 \mathrm{H}, \mathrm{C}\left(\mathrm{CH}_{3}\right)_{2}\right), 1.65-1.74\left(\mathrm{~m}, 1 \mathrm{H}, \mathrm{CHCH}_{2, \mathrm{Lys}}\right), 1.85-1.93\left(\mathrm{~m}, 1 \mathrm{H}, \mathrm{CHCH}_{2, \mathrm{Arg}}\right), 2.07\left(\mathrm{~s}, 3 \mathrm{H}, \mathrm{CH}_{3, \mathrm{Pbf}}\right), 2.44$ (dd, $J=15.7 .6 .0 \mathrm{~Hz}, 1 \mathrm{H}, \mathrm{CHCH}_{2, \mathrm{DPhe}}$ ), 2.53, $2.60\left(2 \mathrm{~s}, \mathrm{CH}_{3, \mathrm{Pht}}\right), 2.72$ (dd, $J=15.7,8.6 \mathrm{~Hz}, 1 \mathrm{H}, \mathrm{CHCH}_{2, \mathrm{DPhe}}$ ), 2.90-2.94 (m, 1 $\mathrm{H}, \mathrm{CHCH}_{2, \mathrm{Asp}}$ ), 3.02 (s, $2 \mathrm{H}, \mathrm{CH}_{2, \text { Pbf }}$ ), 3.04-3.09 (m, $3 \mathrm{H}, \mathrm{CHCH}_{2, \mathrm{Asp}}, \mathrm{CH}_{2} \mathrm{NH}_{\text {Lys }}$ ), 3.16-3.22 (m, $2 \mathrm{H}, \mathrm{CH}_{2} \mathrm{NH}_{\text {Arg }}$ ), 3.35 (dd, $J=$ $\left.15.3,4.0 \mathrm{~Hz}, 1 \mathrm{H}, \mathrm{CH}_{2, \text { Giy }}\right), 4.09-4.14\left(\mathrm{~m}, 1 \mathrm{H}, \mathrm{\alpha}-\mathrm{CH}_{\text {Lys }}\right), 4.18-4.23\left(\mathrm{~m}, 1 \mathrm{H}, \mathrm{\alpha}-\mathrm{CH}_{\text {Arg }}\right), 4.25$ (dd, J $=15.3,7.9 \mathrm{~Hz}, 1 \mathrm{H}, \mathrm{CH}_{2, \text { Giy }}$ ), 4.69 (q, $\left.J=7.6 \mathrm{~Hz}, 1 \mathrm{H}, \alpha-\mathrm{CH}_{\text {Asp }}\right), 4.78$ (dt, $\left.J=8.6,6.0 \mathrm{~Hz}, 1 \mathrm{H}, \mathrm{a}-\mathrm{CH}_{\mathrm{DPhe}}\right), 5.09\left(\mathrm{~s}, 2 \mathrm{H}, \mathrm{CH}_{2} \mathrm{Ar}_{\mathrm{Cbz}}\right), 6.51-7.05\left(\mathrm{~m}, 3 \mathrm{H}, \mathrm{NH}_{\text {Arg }}\right.$ ), $7.11\left(\mathrm{t}, J=5.7 \mathrm{~Hz}, 1 \mathrm{H}, \mathrm{NH}_{\mathrm{Cbz}}\right), 7.20-7.46(\mathrm{~m}, 10 \mathrm{H}, \mathrm{Ar}), 7.95\left(\mathrm{~d}, J=8.7 \mathrm{~Hz}, 1 \mathrm{H}, \mathrm{NH}_{\text {DPhe }}\right), 8.05-8.10\left(\mathrm{~m}, 2 \mathrm{H}, \mathrm{NH}_{\text {Arg, Lys }}\right), 8.20$ (d, $J=7.9 \mathrm{~Hz}, 1 \mathrm{H}, \mathrm{NH}_{\text {Asp }}$ ), 8.30 (dd, $\left.J=7.9,4.0 \mathrm{~Hz}, 1 \mathrm{H}, \mathrm{NH}_{\text {Gly }}\right)$ ppm. ${ }^{13} \mathrm{C}-\mathrm{NMR}\left(150 \mathrm{MHz}, \mathrm{DMF}-\mathrm{D}_{7}\right): \delta=12.9,18.5,19.8$, 24.2, 27.1, 28.4, 29.0, 29.3, 37.7, 38.5, 41.3, 41.6, 43.8, 44.6, 50.6, 53.8, 55.8, 56.0, 66.5, 81.1, 87.4, 117.7, 125.7, 127.5, 128.8, 129.3, 129.5, 130.3, 133.0, 135.8, 138.8, 138.9, 157.6, 157.8, 159.0, 163.2, 170.6, 170.8, 171.8, 172.5, 172.8, 173.5 ppm. HRMS (pos. ESI-TOF): $m / z$ calculated for $\mathrm{C}_{52} \mathrm{H}_{72} \mathrm{~N}_{9} \mathrm{O}_{12} \mathrm{~S}[\mathrm{M}+\mathrm{H}]^{+} 1046.5016$, found 1046.5023 .

\section{$\mathrm{cR}(\mathrm{Pbf}) \mathrm{GD}(t \mathrm{Bu}) \mathrm{fK}(\mathrm{S} 4)$}

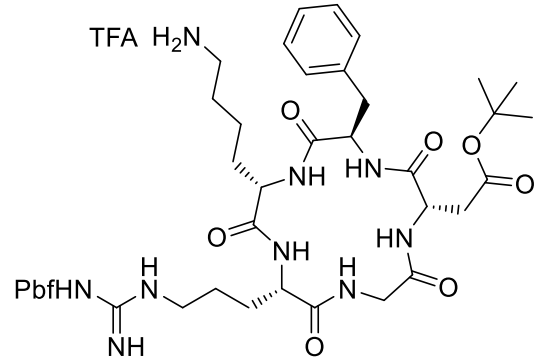

$\mathrm{cR}(\mathrm{Pbf}) \mathrm{GD}(\mathrm{tBu}) \mathrm{fK}(\mathrm{Cbz})(\mathrm{S} 3,114 \mathrm{mg}, 0.11 \mathrm{mmol})$ was dissolved in DMF $(10 \mathrm{ml})$, the solution was charged with 10\% $\mathrm{Pd}$ on carbon, and a balloon with hydrogen gas was installed on the flask allowing to bubble through the solution via syringe needle. The reaction was stirred overnight, filtered over celite and the solvent was evaporated under reduced pressure. The residue was taken up in $\mathrm{H}_{2} \mathrm{O} / \mathrm{ACN}(1: 1,4 \mathrm{ml})$ and a drop of TFA was added. The solution was filtered through a syringe filter and the filtrate was purified by HPLC (15-100\% ACN in $22 \mathrm{~min}, 4 \mathrm{~min} 100 \%)$, yielding $62 \mathrm{mg}(55 \%)$ of the title compound S4 as white powder after lyophilization. ${ }^{1} \mathrm{H}-\mathrm{NMR}\left(600 \mathrm{MHz}, \mathrm{DMF}-\mathrm{D}_{7}\right): \delta=1.25-1.35\left(\mathrm{~m}, 2 \mathrm{H}, \mathrm{CHCH}_{2} \mathrm{CH}_{2, \mathrm{Lys}}\right), 1.39\left(\mathrm{~s}, 9 \mathrm{H}, \mathrm{C}\left(\mathrm{CH}_{3}\right)_{3}\right), 1.45$ (s, $\left.6 \mathrm{H}, \mathrm{C}\left(\mathrm{CH}_{3}\right)_{2}\right), 1.45-1.55$ (m, $\left.2 \mathrm{H}, \mathrm{CHCH}_{2, \text { Lys }}\right), 1.55-1.80$ (m, $5 \mathrm{H}, \mathrm{CHCH}_{2, \text { Arg }}, \mathrm{CH}_{2} \mathrm{CH}_{2} \mathrm{NH}_{\mathrm{Lys}}, \mathrm{CHCH}_{2, \text { Arg }}$ ), 1.87-1.95 (m, $1 \mathrm{H}$, $\mathrm{CHCH}_{2, \mathrm{Arg}}$ ), $2.07\left(\mathrm{~s}, 3 \mathrm{H}, \mathrm{CH}_{3, \mathrm{Pbf}}\right), 2.44$ (dd, $\left.J=15.7 .6 .0 \mathrm{~Hz}, 1 \mathrm{H}, \mathrm{CHCH}_{2, \mathrm{DPhe}}\right), 2.53,2.60$ (2 s, $\left.\mathrm{CH}_{3, \mathrm{Pff}}\right), 2.76$ (dd, $J=15.7,8.6$ $\left.\mathrm{Hz}, 1 \mathrm{H}, \mathrm{CHCH}_{2, \mathrm{DPhe}}\right), 2.89$ (dd, $\left.J=13.6,6.7 \mathrm{~Hz}, 1 \mathrm{H}, \mathrm{CHCH}_{2, \mathrm{Asp}}\right), 2.99-3.07\left(\mathrm{~m}, 2 \mathrm{H}, \mathrm{CH}_{2} \mathrm{NH}_{\mathrm{Lys}}\right), 3.02\left(\mathrm{~s}, 2 \mathrm{H}, \mathrm{CH}_{2, \mathrm{Pbf}}\right), 3.10$ (dd, $J=13.6,7.8 \mathrm{~Hz}, 1 \mathrm{H}, \mathrm{CHCH}_{2, \mathrm{Asp}}$ ), 3.15-3.20 (m, $2 \mathrm{H}, \mathrm{CH}_{2} \mathrm{NH}_{\text {Arg }}$ ), 3.35 (dd, $\left.J=15.5,3.6 \mathrm{~Hz}, 1 \mathrm{H}, \mathrm{CH}_{2, \text { Gly }}\right), 4.20-4.25(\mathrm{~m}, 2$ $\mathrm{H}, \alpha-\mathrm{CH}_{\text {Arg }}, \alpha-\mathrm{CH}_{\mathrm{Lys}}$ ), 4.27 (dd, $J=15.5,8.3 \mathrm{~Hz}, 1 \mathrm{H}, \mathrm{CH}_{2, \mathrm{Gly}}$ ), 4.68 (q, $J=7.5 \mathrm{~Hz}, 1 \mathrm{H}, \alpha-\mathrm{CH}_{\text {Asp }}$ ), 4.81 (dt, $J=8.6,6.0 \mathrm{~Hz}, 1 \mathrm{H}$, $\left.\mathrm{a}-\mathrm{CH}_{\mathrm{DPhe}}\right), 6.65-7.11\left(\mathrm{~m}, 3 \mathrm{H}, \mathrm{NH}_{\text {Arg }}\right), 7.19-7.31(\mathrm{~m}, 5 \mathrm{H}, \mathrm{Ar}), 8.07$ (d, $\left.J=8.8 \mathrm{~Hz}, 1 \mathrm{H}, \mathrm{NH}_{\mathrm{DPhe}}\right), 8.19-8.30$ ( $\mathrm{m}, 3 \mathrm{H}, \mathrm{NH}_{\text {Gly,Arg, Asp }}$, $\mathrm{NH}_{2, \text { Lys }}$ ), 8.44 (s, $1 \mathrm{H}, \mathrm{NH}_{\text {Lys }}$ ) ppm. ${ }^{13} \mathrm{C}-\mathrm{NMR}(150 \mathrm{MHz}$, DMF-D $): \delta=12.9,18.5,19.8,23.8,27.2,27.9,28.4,29.0,29.3,32.7$, 38.0, 38.2, 40.7, 43.8, 44.5, 50.5, 53.6, 55.7, 81.0, 87.5, 116.5, 117.7, 118.5, 125.7, 127.4, 129.3, 130.4, 133.0, 135.7, 137.9, 138.8, 139.2, 157.8, 159.0, 163.2, 170.6, 170.7, 171.7, 172.3, 172.4, 173.4. HRMS (pos. ESI-TOF): $\mathrm{m} / \mathrm{z}$ calculated for $\mathrm{C}_{44} \mathrm{H}_{65} \mathrm{~N}_{9} \mathrm{O}_{10} \mathrm{~S}[\mathrm{M}+\mathrm{H}]^{+}$912.4648, found 912.4645 . 


\section{Synthesis of Dye and Quencher}

\section{Synthesis of Cy3 (S7) ${ }^{3}$}<smiles>CC1=[N+](C)c2ccccc2C1(C)C</smiles><smiles>CC1=[N+](CCCCCC(=O)O)c2ccccc2C1(C)C</smiles>

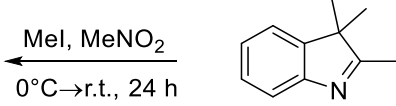

$77 \%$

$$
\mathrm{PhHN} \curvearrowright_{\mathrm{NPh}}
$$

1. $\mathrm{Ac}_{2} \mathrm{O}, 120^{\circ} \mathrm{C}, 30 \mathrm{~min}$

2. pyridine, r.t., $18 \mathrm{~h}$

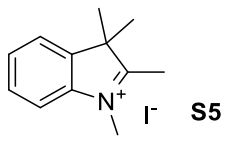

$58 \%$
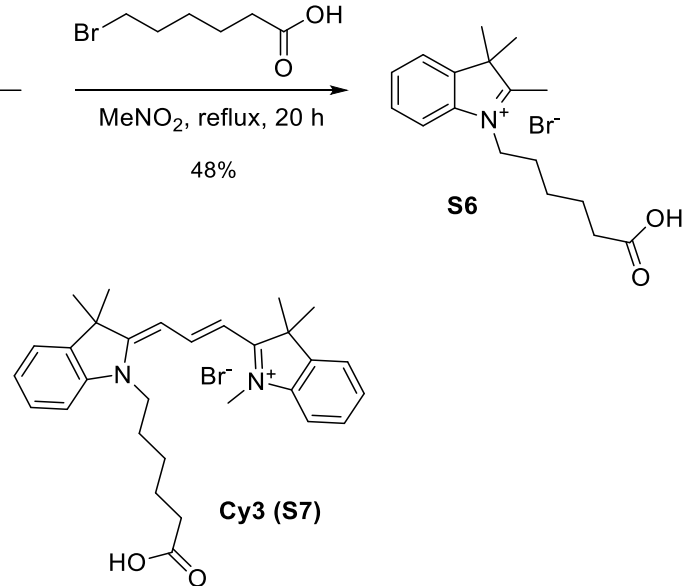

1,2,3,3-Tetramethyl-3H-indolium iodide (S5)<smiles>CC1=[N+]([I-])c2ccccc2C1(C)C</smiles>

2,3,3-Trimethylindolenine $(19.0 \mathrm{mmol}, 3.00 \mathrm{~g})$ was dissolved in nitromethane $(12 \mathrm{ml})$ and iodomethane $(22.8 \mathrm{mmol}, 1.42 \mathrm{ml})$ was added at $0^{\circ} \mathrm{C}$. The solution was allowed to warm up to r.t. and was stirred for $12 \mathrm{~h}$. Diethyl ether (60 ml) was added and the precipitate was filtrated and washed with diethyl ether to afford $4.41 \mathrm{~g} \mathrm{(79 \% )} \mathrm{1,2,3,3-tetramethyl-3H-indolium} \mathrm{iodide} \mathrm{(S5)}$ as slightly pink solid. ${ }^{1} \mathrm{H}-\mathrm{NMR}\left(300 \mathrm{MHz}, \mathrm{DMSO}-\mathrm{D}_{6}\right): \delta=1.52\left(\mathrm{~s}, 6 \mathrm{H}, \mathrm{C}\left(\mathrm{CH}_{3}\right)_{2}\right), 2.76\left(\mathrm{~s}, 3 \mathrm{H}, \mathrm{CCH}_{3}\right), 3.97(\mathrm{~s}, 3 \mathrm{H}, \mathrm{NCH}), 7.56-$ $7.58(\mathrm{~m}, 2 \mathrm{H}, 4,5-\mathrm{H}), 7.78-7.96(\mathrm{~m}, 2 \mathrm{H}, 6,7-\mathrm{H})$ ppm. ${ }^{13} \mathrm{C}-\mathrm{NMR}\left(75 \mathrm{MHz}\right.$, DMSO-D6): $\delta=14.1\left(\mathrm{CCH}_{3}\right), 21.7\left(\mathrm{C}\left(\mathrm{CH}_{3}\right)_{2}\right), 34.7$ $\left(\mathrm{NCH}_{3}\right), 53.9$ (C-3), 115.1 (C-7), 123.3 (C-4), 128.8, 129.3 (C-5,6), 141.6, 142.1 (C-3-C-C-4, C-7-C), 196.0 (C-2) ppm.

\section{1-(5-Carboxypentyl)-2,3,3-trimethyl-3H-indolium bromide (S6)}

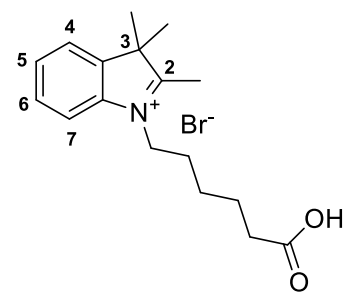

2,3,3-Trimethylindolenine (19.0 mmol, $3.00 \mathrm{~g})$ and 6-bromohexanoic acid (38.0 mmol, $7.41 \mathrm{~g})$ were dissolved in nitromethane $(15 \mathrm{ml})$ and the solution was heated to $80^{\circ} \mathrm{C}$ for $12 \mathrm{~h}$. After cooling down to r.t. diethyl ether $(60 \mathrm{ml})$ was added and the formed precipitate was filtrated and washed with diethyl ether. The product was redissolved in methanol ( $8 \mathrm{ml})$ and diethyl ether (60 $\mathrm{ml}$ ) was added. The precipitate was washed with ethyl acetate and diethyl ether to afford $3.26 \mathrm{~g} \mathrm{(48 \% )} \mathrm{of} \mathrm{the} \mathrm{title} \mathrm{compound}$ S6 as brownish solid. ${ }^{1} \mathrm{H}-\mathrm{NMR}\left(300 \mathrm{MHz}, \mathrm{DMSO}-\mathrm{D}_{6}\right): \delta=1.34-1.62\left(\mathrm{~m}, 4 \mathrm{H}, \mathrm{CH}_{2}\right), 1.54\left(\mathrm{~s}, 6 \mathrm{H}, \mathrm{C}\left(\mathrm{CH}_{3}\right)_{2}\right), 1.77-1.92(\mathrm{~m}, 2 \mathrm{H}$, $\mathrm{CH}_{2}$ ), $2.23\left(\mathrm{t}, J=7.1 \mathrm{~Hz}, 2 \mathrm{H}, \mathrm{CH}_{2} \mathrm{CO}_{2} \mathrm{H}\right), 2.85\left(\mathrm{~s}, 3 \mathrm{H}, \mathrm{CCH}_{3}\right), 4.46\left(\mathrm{t}, J=7.7 \mathrm{~Hz}, 2 \mathrm{H}, \mathrm{NCH}_{2}\right), 7.57-7.68(\mathrm{~m}, 2 \mathrm{H}, 4,5-\mathrm{H}), 7.80-$

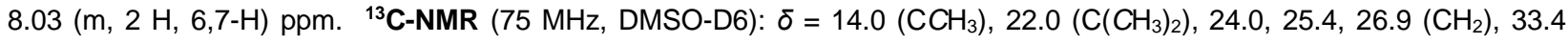
$\left(\mathrm{CH}_{2} \mathrm{CO}_{2} \mathrm{H}\right), 47.4\left(\mathrm{NCH}_{2}\right), 54.2$ (C-3), 115.5 (C-7), 123.5 (C-4), 128.9, 129.4 (C-5,6), 141.0, 141.9 (C-3-C-C-4, C-7-C), 174.3 $\left(\mathrm{CO}_{2} \mathrm{H}\right), 196.5$ (C-2) ppm. 


\section{Cy3 (S7)}

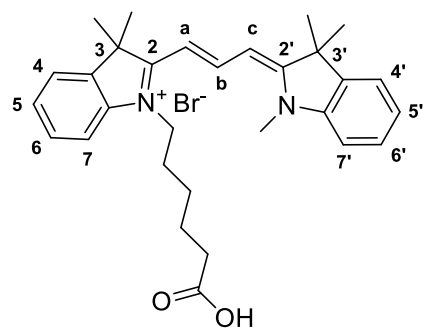

1-(5-Carboxypentyl)-2,3,3-trimethyl-3H-indolium bromide (S6, $500 \mathrm{mg}, 1.41 \mathrm{mmol}$ ) and N,N'-diphenylformamidine (332 mg, $1.69 \mathrm{mmol}$ ) were dissolved in acetic anhydride $(5 \mathrm{ml})$ and heated to $120^{\circ} \mathrm{C}$ for $45 \mathrm{~min}$ in a sealed tube. The solution was cooled to r.t. and 1,2,3,3-tetramethyl-3H-indolium iodide $(\mathbf{S 5}, 595 \mathrm{mg}, 1.97 \mathrm{mmol})$ in dry pyridine $(5 \mathrm{ml})$ was added. The mixture was stirred for $12 \mathrm{~h}$ at r.t., concentrated in vacuo and purified by flash chromatography $\left(\mathrm{SiO}_{2}, \mathrm{DCM}, 1 \% \mathrm{AcOH}, 3-10 \% \mathrm{MeOH}\right)$ to afford $437 \mathrm{mg}(58 \%)$ of the title compound $\mathbf{S} 7$ as dark red foam. ${ }^{1} \mathbf{H}-\mathbf{N M R}\left(300 \mathrm{MHz}, \mathrm{CDCl}_{3}\right): \delta=1.55-1.95\left(\mathrm{~m}, 6 \mathrm{H}, \mathrm{CH}_{2}\right), 1.69$ (bs, $\left.12 \mathrm{H}, \mathrm{C}\left(\mathrm{CH}_{3}\right)_{2}\right), 2.43$ (t, J = 7.0 Hz, $2 \mathrm{H}, \mathrm{CH}_{2} \mathrm{CO}_{2} \mathrm{H}$ ), 3.78 (s, $\left.3 \mathrm{H}, \mathrm{NCH}_{3}\right), 4.17$ (t, J = 7.8 Hz, $\left.2 \mathrm{H}, \mathrm{NCH}_{2}\right), 7.08-7.41(\mathrm{~m}, 10$ $\left.\mathrm{H}, \mathrm{H}_{\mathrm{Ar}}, \mathrm{a}-\mathrm{H}, \mathrm{c}-\mathrm{H}\right), 8.40$ (t, $\left.J=13.4 \mathrm{~Hz}, 1 \mathrm{H}, \mathrm{b}-\mathrm{H}\right) \mathrm{ppm} .{ }^{13} \mathrm{C}-\mathrm{NMR}\left(75 \mathrm{MHz}, \mathrm{CDCl}_{3}\right): \delta=24.6,26.3,27.2\left(\mathrm{CH}_{2}\right), 28.2,28.3$ $\left(\mathrm{C}\left(\mathrm{CH}_{3}\right)_{2}\right), 32.9\left(\mathrm{NCH}_{3}\right), 34.2\left(\mathrm{CH}_{2} \mathrm{CO}_{2} \mathrm{H}\right), 44.8\left(\mathrm{NCH}_{2}\right), 49.0,49.1\left(\mathrm{C}-3,3^{\prime}\right), 104.5,105.0(\mathrm{C}-\mathrm{a}, \mathrm{C}), 111.0,111.1\left(\mathrm{C}-7,7^{\prime}\right), 122.2$, 122.3 (C-4,4'), 125.5 (C-5,5'), 129.0, 129.1 (C-6,6'), 140.6, 140.7 (C-3-C-C-4, C-3'-C-C-4'), 142.1, 142.8 (C-7-C-N, C-7'-C-N), 150.8 (C-b), 173.7, 174.4 (C-2,2'), $177.7\left(\mathrm{CO}_{2} \mathrm{H}\right)$ ppm. HRMS (pos. ESI-TOF): $\mathrm{m} / \mathrm{z}$ calculated for [M] ${ }^{+}$457.2850, found 457.2851.

\section{Cy3-EDA (S8)}

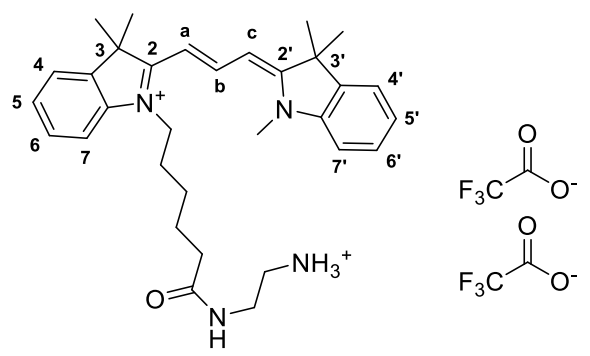

Cy3 (S7, $100 \mathrm{mg}, 0.19 \mathrm{mmol})$ was dissolved in acetonitrile $(5 \mathrm{ml})$ and DIPEA ( $65 \mu \mathrm{l}, 0.37 \mathrm{mmol})$ and HATU (71 mg, $0.19 \mathrm{mmol})$ were added. The solution was stirred for $10 \mathrm{~min}$, then tert-butyl (2-aminoethyl)carbamate (36 mg, $0.22 \mathrm{mmol})$ was added and the reaction was stirred at r.t. overnight. The product was then diluted with DCM $(20 \mathrm{ml})$ and washed with water. The organic layer was dried over $\mathrm{MgSO}_{4}$, filtered and the solvent was evaporated under reduced pressure. Flash chromatography $\left(\mathrm{SiO}{ }_{2}\right.$, DCM, 3\% MeOH) afforded $111 \mathrm{mg}(88 \%)$ Cy3-EDABoc (S8a) as dark red foam. ${ }^{1} \mathbf{H}-\mathbf{N M R}\left(300 \mathrm{MHz}, \mathrm{CDCl}_{3}\right): \delta=1.39(\mathrm{~s}, 9 \mathrm{H}$, $\left.\mathrm{C}\left(\mathrm{CH}_{3}\right)_{3}\right), 1.55\left(\mathrm{~m}_{\mathrm{c}}, 2 \mathrm{H}, \mathrm{CH}_{2}\right), 1.71,1.72\left(2 \mathrm{~s}, 12 \mathrm{H}, \mathrm{C}\left(\mathrm{CH}_{3}\right)_{2}\right), 1.72\left(\mathrm{~m}_{\mathrm{c}}, 2 \mathrm{H}, \mathrm{CH}_{2}\right), 1.82\left(\mathrm{~m}_{\mathrm{c}}, 2 \mathrm{H}, \mathrm{CH}_{2}\right), 2.26(\mathrm{t}, J=7.2 \mathrm{~Hz}, 2$ $\left.\mathrm{H}, \mathrm{CH}_{2} \mathrm{CO}\right)$, 3.22, $3.32\left(2 \mathrm{~m}_{\mathrm{c}}, 4 \mathrm{H}, \mathrm{NHCH}_{2} \mathrm{CH}_{2}\right), 3.66$ (s, $\left.3 \mathrm{H}, \mathrm{NCH}_{3}\right), 4.02\left(\mathrm{t}, J=7.8 \mathrm{~Hz}, 2 \mathrm{H}, \mathrm{NCH}_{2}\right), 5.23\left(\mathrm{bs}, 1 \mathrm{H}, \mathrm{NH}_{\mathrm{Boc}}\right), 6.45$ (d, $J=13.5 \mathrm{~Hz}, \mathrm{a}-\mathrm{H}), 6.48(\mathrm{~s}, 1 \mathrm{H}, \mathrm{NH}), 6.55(\mathrm{~d}, J=13.5 \mathrm{~Hz}, 1 \mathrm{H}, \mathrm{c}-\mathrm{H}), 7.09-7.16\left(\mathrm{~m}, 2 \mathrm{H}, 5,5^{\prime}-\mathrm{H}\right), 7.21-7.30\left(\mathrm{~m}, 2 \mathrm{H}, 7,7^{\prime}-\mathrm{H}\right)$, 7.34-7.44 (m, $4 \mathrm{H}, 4,4^{\prime}, 6,6$ '-H), 8.39 (t, $\left.J=13.5 \mathrm{~Hz}, 1 \mathrm{H}, \mathrm{b}-\mathrm{H}\right)$ ppm. ${ }^{13} \mathrm{C}-\mathrm{NMR}\left(75 \mathrm{MHz}, \mathrm{CDCl}_{3}\right): \delta=25.4\left(\mathrm{CH}_{2}\right), 26.2$ $\left(\mathrm{CH}_{2} \mathrm{CH}_{2} \mathrm{CO}\right), 27.2\left(\mathrm{CH}_{2} \mathrm{CH}_{2} \mathrm{~N}\right)$, 28.1, $28.2\left(\mathrm{C}\left(\mathrm{CH}_{3}\right)_{2}\right), 28.5\left(\mathrm{C}\left(\mathrm{CH}_{3}\right)_{3}\right), 31.5\left(\mathrm{NCH}_{3}\right), 36.3\left(\mathrm{CH}_{2} \mathrm{CO}\right), 40.0,40.9\left(\mathrm{NHCH}_{2} \mathrm{CH}_{2}\right)$, 44.6 $\left(\mathrm{CH}_{2} \mathrm{~N}\right), 49.1,49.3$ (C-3,3'), 103.4, 103.9 (C-a,c), 111.0, 111.1 (C-5,5'), 122.2, 122.3 (C-6,6'), 125.6 (C-7,7'), 129.0, 129.1 (C-4,4'), 140.5, 140.6 (C-C-4, C-C-4'), 142.0 (C-C-7), 142.7 (C-C-7'), 150.8 (C-b), 156.5 (NHCOO), 174.1, 174.3 (C-2,2'), 174.8 (NHCO) ppm. HRMS (pos. ESI-TOF): $m / z$ calculated for $\mathrm{C}_{37} \mathrm{H}_{51} \mathrm{~N}_{4} \mathrm{O}_{3}[\mathrm{M}]^{+} 599.3961$, found 599.3954.

Cy3-EDABoc (S8a, $111 \mathrm{mg}, 0.16 \mathrm{mmol})$ was dissolved in DCM/TFA (10 ml, 1:1) and stirred for $30 \mathrm{~min}$ at r.t. The solvent was evaporated under reduced pressure and to yield $114 \mathrm{mg}$ (quant.) Cy3-EDA (S8) as dark red oil. HRMS (pos. ESI-TOF): $\mathrm{m} / z$ calculated for $\mathrm{C}_{32} \mathrm{H}_{43} \mathrm{~N}_{4} \mathrm{O}[\mathrm{M}]^{+} 499.3437$, found 499.3447 . 
Synthesis of BHQ-2 (S10)

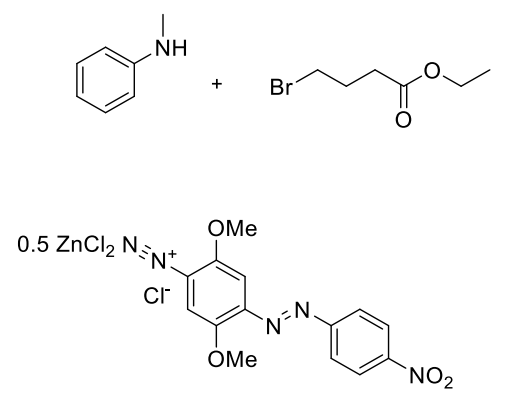

1. 2,6-lutidine, $\mathrm{CH}_{3} \mathrm{CN}$, reflux, $18 \mathrm{~h}$

2. $\mathrm{NaOH}$, dioxane/ $/ \mathrm{H}_{2} \mathrm{O}$, reflux, $4 \mathrm{~h}$

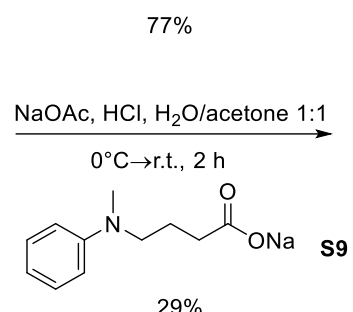

$29 \%$

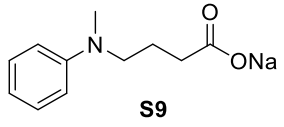

s9

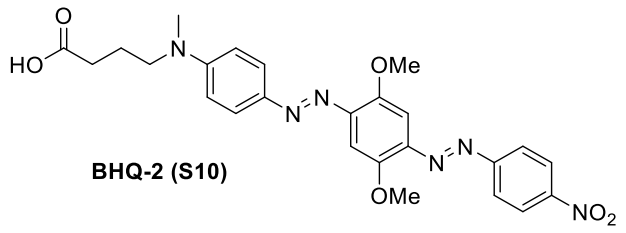

Sodium 4-(methyl(phenyl)amino)butanoate (S9) ${ }^{5}$<smiles>CN(CCCC(=O)O[Na])c1ccccc1</smiles>

$\mathrm{N}$-methylaniline $(2.50 \mathrm{ml}, 23.3 \mathrm{mmol}), 4$-bromobutyric acid ethyl ester (3.68 ml, $25.7 \mathrm{mmol})$ and 2,6-lutidine (2.96 ml, 25.7 $\mathrm{mmol})$ were dissolved in acetonitrile $(30 \mathrm{ml})$ and the mixture was refluxed for $20 \mathrm{~h}$. The solvent was evaporated under reduced pressure and the residue was partitioned between $\mathrm{H}_{2} \mathrm{O}$ and EtOAc. The org. phase was separated, dried over $\mathrm{MgSO}_{4}$ and the solvents were evaporated under reduced pressure. The crude was purified by flash chromatography $(\mathrm{SiO}, \mathrm{cHex} / \mathrm{EtOAc}=$ $10: 1)$ to yield $4.78 \mathrm{~g}$ ethyl 4 -(methyl(phenyl)amino)butanoate $(93 \%)$ as colorless oil. The compound $(4.79 \mathrm{~g}, 21.6 \mathrm{mmol})$ was dissolved in dioxane $(12 \mathrm{ml})$ and sodium hydroxide $(1.31 \mathrm{~g}, 32.4 \mathrm{mmol})$ in water $(12 \mathrm{ml})$ was added. The reaction was stirred for $48 \mathrm{~h}$ at r.t. The solution was acidified to about $\mathrm{pH} 4$ by addition of $2 \mathrm{M}$ aq. $\mathrm{HCl}$ and extracted 3 times with EtOAc. The combined org. phases were dried over $\mathrm{MgSO}_{4}$, filtered and the solvent evaporated under reduced pressure to yield $3.37 \mathrm{~g}$ (81\%) 4-(methyl(phenyl)amino)butanoic acid as colorless oil. The product was transformed into the corresponding sodium salt S9 by stirring in a $1 \mathrm{M}$ aq. $\mathrm{NaOH}$ (slight excess) for $3 \mathrm{~h}$ at r.t., evaporating the water and eventually precipitating the product in acetonitrile. ${ }^{1} \mathrm{H}-\mathrm{NMR}\left(300 \mathrm{MHz}, \mathrm{CD}_{3} \mathrm{CN}\right): \delta=1.80$ (tt, $\left.J=7.3 \mathrm{~Hz}, 2 \mathrm{H}, \mathrm{CH}_{2} \mathrm{CH}_{2} \mathrm{CH}_{2} \mathrm{CO}_{2} \mathrm{H}\right), 2.31\left(\mathrm{t}, J=7.3 \mathrm{~Hz}, 2 \mathrm{H}, \mathrm{CH}_{2} \mathrm{CO}{ }_{2} \mathrm{H}\right)$, $2.89\left(\mathrm{~s}, 3 \mathrm{H}, \mathrm{CH}_{3}\right), 3.33\left(\mathrm{t}, J=7.3 \mathrm{~Hz}, 2 \mathrm{H}, \mathrm{NCH}_{2}\right), 6.63\left(\mathrm{t}, J=7.3 \mathrm{~Hz}, 1 \mathrm{H}, p-\mathrm{CH}_{\mathrm{Ar}}\right), 6.70-6.78\left(\mathrm{~m}_{\mathrm{c}}, 2 \mathrm{H}, 0-\mathrm{CH}_{\mathrm{Ar}}\right), 7.14-7.23\left(\mathrm{~m}_{\mathrm{c}}\right.$, $\left.2 \mathrm{H}, m-\mathrm{CH}_{\mathrm{Ar}}\right)$ ppm. ${ }^{13} \mathrm{C}-\mathrm{NMR}\left(75 \mathrm{MHz}, \mathrm{CD}_{3} \mathrm{CN}\right): \delta=22.4\left(\mathrm{CH}_{2} \mathrm{CH}_{2} \mathrm{CH}_{2} \mathrm{CO}_{2} \mathrm{H}\right), 31.3\left(\mathrm{CH}_{2} \mathrm{CO}{ }_{2} \mathrm{H}\right), 38.5\left(\mathrm{CH}_{3}\right), 52.3\left(\mathrm{NCH}_{2}\right), 113.1$ $\left(o-\mathrm{CH}_{\mathrm{Ar}}\right), 116.8\left(p-\mathrm{CH}_{\mathrm{Ar}}\right), 130.0\left(m-\mathrm{CH}_{\mathrm{Ar}}\right), 150.4\left(\mathrm{~N}-\mathrm{C}_{\mathrm{Ar}}\right), 174.9\left(\mathrm{CO}_{2} \mathrm{H}\right)$ ppm. HRMS (pos. ESI-TOF): $\mathrm{m} / z$ calculated for $\mathrm{C}_{11} \mathrm{H}_{16} \mathrm{NO}_{2}[\mathrm{M}+\mathrm{H}]^{+}$194.1176, found 194.1177.

\section{BHQ-2 (S10)}<smiles>COc1cc(N=Nc2ccc([N+](=O)[O-])cc2)c(OC)cc1N=Nc1ccc(N(C)CCCC(=O)O)cc1</smiles>

Fast Black K salt $(2.94 \mathrm{~g}, 7.04 \mathrm{mmol})$ was suspended in $\mathrm{H}_{2} \mathrm{O}(30 \mathrm{ml})$ at $0^{\circ} \mathrm{C}$. Sodium 4-(methyl(phenyl)amino)butanoate (S9, $0.76 \mathrm{~g}, 3.52 \mathrm{mmol})$, sodium acetate $(1.85 \mathrm{~g}, 22.53 \mathrm{mmol})$ and $37 \%$ aq. $\mathrm{HCl}(0.9 \mathrm{ml})$ dissolved in $\mathrm{H}_{2} \mathrm{O} /$ acetone $(60 \mathrm{ml}, 1: 1)$ were added dropwise during $1 \mathrm{~h}$ at $0^{\circ} \mathrm{C}$. The reaction mixture was then allowed to warm up to r.t. and was stirred for an additional hour. The mixture was extracted with $\mathrm{DCM}$ and the combined org. phases were washed with brine and dried over $\mathrm{MgSO}_{4}$. The crude was purified via flash chromatography $\left(\mathrm{SiO}_{2}, \mathrm{DCM}, 1.5 \% \mathrm{MeOH}, 2 \% \mathrm{AcOH}\right)$ to afford $0.52 \mathrm{~g}(29 \%) \mathrm{BHQ}-2(\mathrm{~S} 10)$ as dark purple powder. ${ }^{1} \mathrm{H}-\mathrm{NMR}\left(300 \mathrm{MHz}, \mathrm{DMF}-\mathrm{D}_{7}\right): \delta=1.94\left(\mathrm{~m}_{\mathrm{c}}, 2 \mathrm{H}, \mathrm{CH}_{2} \mathrm{CH}_{2} \mathrm{CH}_{2} \mathrm{CO}_{2} \mathrm{H}\right), 2.43\left(\mathrm{t}, J=7.1 \mathrm{~Hz}, 2 \mathrm{H}, \mathrm{CH}_{2} \mathrm{CO}_{2} \mathrm{H}\right), 3.17$ $\left(\mathrm{s}, 3 \mathrm{H}, \mathrm{NCH}_{3}\right), 3.61$ (t, $\left.J=7.3 \mathrm{~Hz}, 2 \mathrm{H}, \mathrm{NCH}_{2}\right), 4.05,4.10\left(2 \mathrm{~s}, 6 \mathrm{H}, \mathrm{OCH}_{3}\right), 7.00$ (dd, J = 9.3, $\left.2.4 \mathrm{~Hz}, 2 \mathrm{H}, 2,6-\mathrm{H}\right), 7.53(\mathrm{~d}, J=$ $2.3 \mathrm{~Hz}, 1 \mathrm{H}, 2^{\prime}-\mathrm{H}$ ), 7.58 (d, J = 2.3 Hz, $1 \mathrm{H}, 5^{\prime}-\mathrm{H}$ ), 7.90 (dd, J = 9.3, $\left.2.4 \mathrm{~Hz}, 2 \mathrm{H}, 3,5-\mathrm{H}\right), 8.14$ (dd, J = 9.0, 2.4 Hz, 2 H, 2",6"'$\mathrm{H}$ ), 8.52 (dd, $J=9.0,2.4 \mathrm{~Hz}, 2 \mathrm{H}, 3$ ", $\left.5^{\prime \prime}-\mathrm{H}\right)$ ppm. ${ }^{13} \mathrm{C}-\mathrm{NMR}\left(75 \mathrm{MHz}, \mathrm{DMF}-\mathrm{D}_{7}\right): \delta=23.2\left(\mathrm{CH}_{2} \mathrm{CH}_{2} \mathrm{CH}_{2} \mathrm{CO}_{2} \mathrm{H}\right), 31.7\left(C \mathrm{C}_{2} \mathrm{CO}_{2} \mathrm{H}\right)$, $39.1\left(\mathrm{NCH}_{3}\right), 52.4\left(\mathrm{NCH}_{2}\right), 57.4,57.5\left(\mathrm{OCH}_{3}\right), 101.3,102.1\left(\mathrm{C}-2^{\prime}, 5^{\prime}\right), 112.7(\mathrm{C}-2,6), 124.6\left(\mathrm{C}-2\right.$ 2", 6”) $^{\prime \prime}, 126.2\left(\mathrm{C}-3^{\prime \prime}, 5^{\prime \prime}\right), 126.9(\mathrm{C}-$ 3,5), 143.1, 147.8 (C-1',4'), 145.1 (C-4), 149.6 (C-4”), 152.0, 154.7 (C-3',6'), 153.5 (C-1), 157.3 (C-1'), 175.3 (CO $\left.{ }_{2} \mathrm{H}\right)$ ppm. HRMS (pos. ESI-TOF): $m / z$ calculated for $\mathrm{C}_{25} \mathrm{H}_{27} \mathrm{~N}_{6} \mathrm{O}_{6}[\mathrm{M}+\mathrm{H}]^{+} 507.1987$, found 507.1981. 
BHQ-2-EDA (S11)

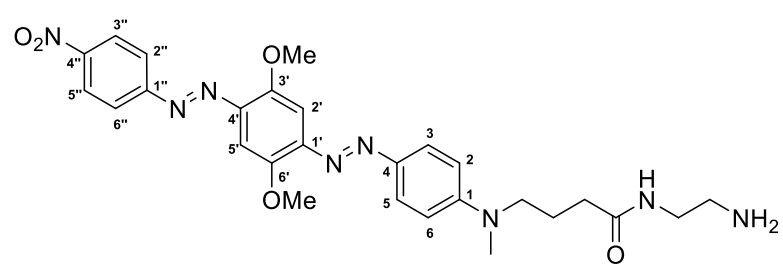

BHQ-2 (S10, $80 \mathrm{mg}, 0.16 \mathrm{mmol})$ was dissolved in dry DMF and DIPEA (55 $\mu \mathrm{l}, 0.32 \mathrm{mmol}$ ) and HATU (60 mg, $0.16 \mathrm{mmol})$ were added. The solution was stirred for $10 \mathrm{~min}$ at r.t., then tert-butyl (2-aminoethyl)carbamate hydrochloride (33 mg, $0.21 \mathrm{mmol})$ was added and the mixture was stirred for $4 \mathrm{~h}$ at r.t. The product was diluted with $\mathrm{DCM}$, washed with water, dried over $\mathrm{MgSO}_{4}$ and filtered. The solvent was evaporated under reduced pressure to afford $96 \mathrm{mg}$ (94\%) BHQ-2-EDABoc (S11a) as dark purple solid. ${ }^{1} \mathrm{H}-\mathrm{NMR}\left(300 \mathrm{MHz}, \mathrm{CDCl}_{3}\right): \delta=1.42\left(\mathrm{~s}, 9 \mathrm{H}, \mathrm{C}\left(\mathrm{CH}_{3}\right)_{3}\right), 1.96\left(\mathrm{~m}_{\mathrm{c}}, 2 \mathrm{H}, \mathrm{CH}_{2} \mathrm{CH}_{2} \mathrm{CH}_{2} \mathrm{CO}_{2} \mathrm{H}\right), 2.26(\mathrm{t}, J=7.1 \mathrm{~Hz}, 2 \mathrm{H}$, $\left.\mathrm{CH}_{2} \mathrm{CO}_{2} \mathrm{H}\right), 3.11\left(\mathrm{~s}, 3 \mathrm{H}, \mathrm{NCH}_{3}\right), 3.28,3.35\left(2 \mathrm{~m}_{\mathrm{c}}, 4 \mathrm{H}, \mathrm{NHCH}_{2} \mathrm{CH}_{2}\right), 3.52\left(\mathrm{t}, J=7.3 \mathrm{~Hz}, 2 \mathrm{H}, \mathrm{NCH}_{2}\right), 4.05,4.10(2 \mathrm{~s}, 6 \mathrm{H}, \mathrm{OCH})$, $4.94\left(\mathrm{~m}_{\mathrm{c}}, 1 \mathrm{H}, \mathrm{NH}\right), 6.40\left(\mathrm{~m}_{\mathrm{c}}, 1 \mathrm{H}, \mathrm{NH}\right), 6.81(\mathrm{~d}, J=8.8 \mathrm{~Hz}, 2 \mathrm{H}, 2,6-\mathrm{H}), 7.47\left(\mathrm{~s}, 1 \mathrm{H}, 2^{\prime}-\mathrm{H}\right), 7.50(\mathrm{~s}, 1 \mathrm{H}, 5$ '- $\mathrm{H}), 7.94(\mathrm{~d}, J=8.8$ $\mathrm{Hz}, 2 \mathrm{H}, 3,5-\mathrm{H}), 8.04$ (d, J = 8.7 Hz, $2 \mathrm{H}, 2$ ", 6"-H), 8.37 (d, J = 8.7 Hz, $2 \mathrm{H}, 3$ ", 5"'-H) ppm. ${ }^{13} \mathrm{C}-\mathrm{NMR}(75 \mathrm{MHz}, \mathrm{CDCl}): \delta=22.9$ $\left(\mathrm{CH}_{2} \mathrm{CH}_{2} \mathrm{CH}_{2} \mathrm{CO}_{2} \mathrm{H}\right), 28.5\left(\mathrm{C}\left(\mathrm{CH}_{3}\right)_{3}\right), 33.3\left(\mathrm{CH}_{2} \mathrm{CO}_{2} \mathrm{H}\right), 39.2\left(\mathrm{NCH}_{3}\right), 40.3,41.4\left(\mathrm{NHCH}_{2} \mathrm{CH}_{2}\right), 52.4\left(\mathrm{NCH}_{2}\right), 56.9\left(\mathrm{OCH}_{3}\right), 80.1$ (C( $\left.\left(\mathrm{CH}_{3}\right)_{3}\right), 100.2,101.2$ (C-2',5'), 123.7 (C-2",6"'), 124.9 (C-3",5”), 126.7 (C-3,5), 142.3, (C-1'), 145.0 (C-4), 146.3 (C-4'), 148.6 (C-4"), 150.9 (C-3'), 152.0 (C-1, barely visible), 153.7 (C-1), 156.6 (C-6'), $157.4\left(\mathrm{NHCO}_{2}\right), 173.0(\mathrm{CONH}), 173.6(\mathrm{NHCO}) \mathrm{ppm}$. HRMS (pos. ESI-TOF): $m / z$ calculated for $\mathrm{C}_{32} \mathrm{H}_{41} \mathrm{~N}_{8} \mathrm{O}_{7}[\mathrm{M}+\mathrm{H}]^{+} 649.3093$, found 649.3081 .

BHQ-2-EDABoc (S11a, 96 mg, $0.15 \mathrm{mmol}$ ) was dissolved in DCM/TFA (10 ml, 1:1) and the solution was stirred for $1 \mathrm{~h}$ at $\mathrm{r.t}$. The solvents were evaporated under reduced pressure and the residue was redissolved in $\mathrm{DCM}$, washed with sat. aq. $\mathrm{NaHCO}_{3}$, and water. The organic layer was dried over $\mathrm{MgSO}_{4}$, filtered, and evaporated under reduced pressure. The residue was taken up in $\mathrm{H}_{2} \mathrm{O} / \mathrm{ACN}$ and lyophilized to yield $80 \mathrm{mg}$ (96\%) BHQ-2-EDA (S11) as dark purple powder. HRMS (pos. ESI-TOF): m/z calculated for $\mathrm{C}_{27} \mathrm{H}_{33} \mathrm{~N}_{8} \mathrm{O}_{5}[\mathrm{M}+\mathrm{H}]^{+} 549.2568$, found 549.2551.

\section{Assembly of Sequence-Quencher Module (S12)}<smiles>NCCCC[C@H](NC(=O)[C@H](CC1CCCCC1)NC(=O)N1CCOCC1)C(=O)NCC(=O)O</smiles>

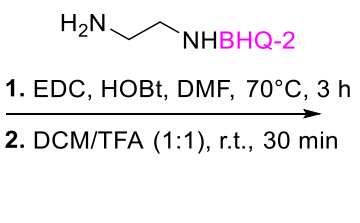<smiles>NCCCC[C@H](NC(=O)[C@H](CC1CCCCC1)NC(=O)N1CCOCC1)C(=O)NCC(=O)NCCN</smiles>

CatS Sequence-BHQ-2 (S12)<smiles>COc1cc(N=Nc2ccc([N+](=O)[O-])cc2)c(OC)cc1N=Nc1ccc(N(C)CCCC(=O)NCCNC(=O)CNC(=O)[C@H](CCCCN)NC(=O)[C@H](CC2CCCCC2)NC(=O)N2CCOCC2)cc1</smiles>

CatS Sequence (S1, $66 \mathrm{mg}, 0.12 \mathrm{mmol}$ ), hydroxybenzotriazole monohydrate (18 $\mathrm{mg}, 0.12 \mathrm{mmol})$, diisopropylethylamine (61 $\mu \mathrm{l}, 0.35 \mathrm{mmol})$, 1-ethyl-3-(3-dimethylaminopropyl)carbodiimide (20 $\mu \mathrm{l}, 0.12 \mathrm{mmol})$ and BHQ-2-EDA (S11, $70 \mathrm{mg}, 0.13 \mathrm{mmol})$ were dissolved in dry DMF $(8 \mathrm{ml})$ and the mixture was heated to $70^{\circ} \mathrm{C}$ for $3 \mathrm{~h}$. DCM was added and the org. layer was washed with $0.5 \mathrm{M} \mathrm{HCl}$, sat. aq. $\mathrm{NaHCO}_{3}$, and brine to yield $93 \mathrm{mg}(73 \%)$ of the Boc-protected peptide-BHQ-2 adduct S12a. ${ }^{1} \mathbf{H}-\mathbf{N M R}$ $\left(600 \mathrm{MHz}, \mathrm{DMF}-\mathrm{D}_{7}\right): \delta=0.83-1.92\left(\mathrm{~m}, 19 \mathrm{H}, \mathrm{CH}_{2}\right.$ Lys $, \mathrm{CH}, \mathrm{CH}_{2}$ cHexAla $), 1.40\left(\mathrm{~s}, 9 \mathrm{H}, \mathrm{C}\left(\mathrm{CH}_{3}\right)_{3}\right), 1.93(\mathrm{tt}, \mathrm{J}=7.2 \mathrm{~Hz}, 2 \mathrm{H}, \mathrm{b}-\mathrm{H}), 2.30$ (t, $J=7.2 \mathrm{~Hz}, 2 \mathrm{H}, \mathrm{c}-\mathrm{H}), 3.03\left(\mathrm{dt}, J=6.6 \mathrm{~Hz}, 2 \mathrm{H}, \mathrm{Gly}-\mathrm{NHCH}_{2} \mathrm{CH}_{2}\right.$ ), 3.17 (s, $3 \mathrm{H}, \mathrm{NCH}_{3}$ ), 3.24-3.36 (m, $4 \mathrm{H}, \mathrm{NHCH}$ Lys, a-H), 3.37-3.48 (m, $\left.4 \mathrm{H}, \mathrm{N}\left(\mathrm{CH}_{2}\right)_{2}\right)$, 3.56-3.64 (m, $6 \mathrm{H}, \mathrm{O}\left(\mathrm{CH}_{2}\right)_{2}$, Gly- $\left.\mathrm{NHCH}_{2} \mathrm{CH}_{2}\right), 3.71(\mathrm{dd}, J=16.6,5.6 \mathrm{~Hz}, 1 \mathrm{H}, \mathrm{CH}$ Gly $), 3.88(\mathrm{dd}, J$ = 16.6. $\left.6.4 \mathrm{~Hz}, 1 \mathrm{H}, \mathrm{CH}_{\mathrm{Gly}}^{\prime}\right), 4.06,4.11\left(2 \mathrm{~s}, 6 \mathrm{H}, \mathrm{OCH}_{3}\right), 4.17-4.24\left(\mathrm{~m}, 1 \mathrm{H}, \mathrm{CH}_{\mathrm{cHexAla}}\right), 4.22-4.31\left(\mathrm{~m}, 1 \mathrm{H}, \mathrm{CH}_{\mathrm{Lys}}\right), 6.69(\mathrm{t}, J=6.1$ 
$\mathrm{Hz}, 1 \mathrm{H}, \mathrm{NH}_{\text {Boc }}$ ), 6.72 (d, J = 6.8 Hz, $\left.1 \mathrm{H}, \mathrm{NH}_{\mathrm{cHexAla}}\right), 7.00$ (d, J = 9.1 Hz, $\left.2 \mathrm{H}, 2,6-\mathrm{H}\right), 7.54,7.59$ (2s, $\left.2 \mathrm{H}, 2^{\prime}, 5^{\prime}-\mathrm{H}\right), 7.69-7.74(\mathrm{~m}$, $\left.1 \mathrm{H}, \mathrm{NH}_{\text {Gly }}\right), 7.90\left(1 \mathrm{H}\right.$, signal overlaid by 3,5-H, $\left.\mathrm{NH}_{\text {linker }}\right), 7.91(\mathrm{~d}, \mathrm{~J}=9.1 \mathrm{~Hz}, 2 \mathrm{H}, 3,5-\mathrm{H}), 8.16(\mathrm{~d}, \mathrm{~J}=8.9 \mathrm{~Hz}, 2 \mathrm{H}, 2$ ", 6"'- $\mathrm{H})$, $8.25\left(\mathrm{t}, J=6.0 \mathrm{~Hz}, 1 \mathrm{H}, \mathrm{NH}_{\text {linker }}\right), 8.30$ (d, J = 6.8 Hz, $\left.1 \mathrm{H}, \mathrm{NH}_{\text {Lys }}\right), 8.53\left(\mathrm{~d}, J=8.9 \mathrm{~Hz}, 2 \mathrm{H}, 3^{\prime \prime}, 5{ }^{\prime \prime}-\mathrm{H}\right) \mathrm{ppm} .{ }^{13} \mathrm{C}-\mathrm{NMR}(150 \mathrm{MHz}$, DMF-D $\left.\mathrm{D}_{7}\right): \delta=22.9$ (C-a), 23.5, 26.3, 26.4, 26.7, 30.0, 30.9, 32.6, 33.8, 34.3, $39.3\left(\mathrm{CH}_{\mathrm{cHexAla}}, \mathrm{CH}_{2, \mathrm{cHexAla}}, \mathrm{CH} 2, \mathrm{Lys}\right), 28.2\left(\mathrm{C}\left(\mathrm{CH}_{3}\right)_{3}\right)$, $32.8(\mathrm{C}-\mathrm{C}), 38.3\left(\mathrm{NCH}_{3}\right), 38.9\left(\mathrm{NHCH}_{2}\right.$ Lys $), 39.1$ (C-a), $40.5\left(\mathrm{Gly}-\mathrm{NHCH}_{2} \mathrm{CH}_{2}\right), 42.9\left(\mathrm{CH}_{2}\right.$ Gly $), 44.6\left(\mathrm{~N}\left(\mathrm{CH}_{2}\right)_{2}\right), 51.9(\mathrm{Gly}-$

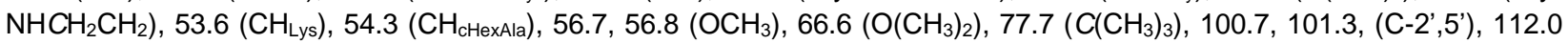
(C-2,6), 123.9 (C-2",6"), 125.4 (C-3",5”), 126.2 (C-3,5), 142.3 (C-1'), 144.4 (C-4), 147.1 (C-4'), 148.8 (C-4”), 151.3 (C-3'), 152.8 (C-1), 153.9 (C-6'), $156.3\left(\mathrm{NHCO}_{2}\right), 156.6$ (C-1'), $158.6(\mathrm{NCONH}), 169.5\left(\mathrm{CONH}_{\text {Lys }}\right), 172.5(\mathrm{C}-\mathrm{C}-\mathrm{CONH}), 172.6$ $\left(\mathrm{CONH}_{\mathrm{Gly}}\right), 175.3\left(\mathrm{CONH}_{\mathrm{cHexAla}}\right)$ ppm. HRMS (pos. ESI-TOF): $\mathrm{m} / z$ calculated for $\mathrm{C}_{54} \mathrm{H}_{78} \mathrm{~N}_{13} \mathrm{O}_{12}[\mathrm{M}+\mathrm{H}]^{+} 1100.5887$, found 1100.5894.

Boc-protected CatS sequence-BHQ-2 (S12a, $81 \mathrm{mg}, 0.09 \mathrm{mmol})$ was dissolved in DCM/TFA $(10 \mathrm{ml}, 1: 1)$ and stirred for $1 \mathrm{~h}$ at r.t. The solvents were evaporated under reduced pressure and the residue was taken up in $\mathrm{H}_{2} \mathrm{O} / \mathrm{ACN}$ and lyophilized to yield $83 \mathrm{mg}$ (quant.) CatS Sequence-BHQ-2 (S12) as dark purple powder. HRMS (pos. ESI-TOF): m/z calculated for $\mathrm{C}_{49} \mathrm{H}_{70} \mathrm{~N}_{13} \mathrm{O}_{10}$ $[\mathrm{M}+\mathrm{H}]^{+} 1000.5363$, found 1000.5363 .

\section{PEG linker Synthesis 6}<smiles>CC(C)(CO)COC(C)(C)CO</smiles>

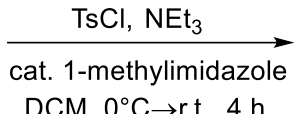

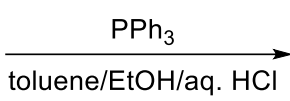
r.t., $24 \mathrm{~h}$

$89 \%$<smiles>CC(C)(C)CNCC(C)(C)COCC(C)(C)CN</smiles>

$\mathbf{s} 17$ $\mathrm{DCM}, 0^{\circ} \mathrm{C} \rightarrow$ r.t., $4 \mathrm{~h}$

$73 \%$

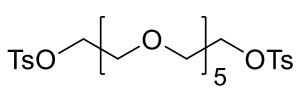

S13<smiles>CC(N)COC(C)CCN</smiles>

S15

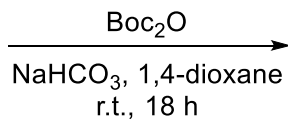

$85 \%$

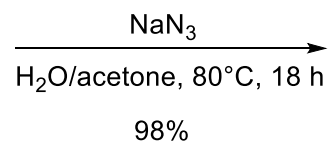<smiles>CC(CN)COCC(C)CN</smiles>

S14

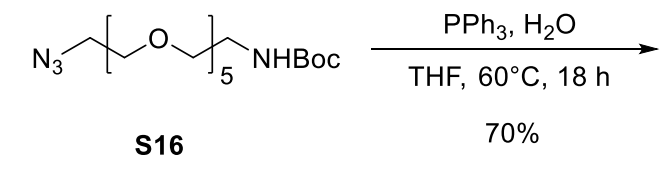

Hexaethylene glycol ditosylate (S13)<smiles>CC(C)(C[SeH])COCC(C)(C)CO[AsH3]</smiles>

Hexaethylene glycol $(3.00 \mathrm{~g}, 10.6 \mathrm{mmol})$ was dissolved in dry DCM $(30 \mathrm{ml})$ and triethylamine $(3.98 \mathrm{ml}, 28.7 \mathrm{mmol})$ and cat. 1methylimidazole $(18 \mathrm{mg}, 0.21 \mathrm{mmol})$ were added. The solution was cooled to $0^{\circ} \mathrm{C}$ and toluenesulfonyl chloride $(5.47 \mathrm{~g}$, $28.7 \mathrm{mmol})$ dissolved in DCM $(20 \mathrm{ml})$ was added dropwise during the course of $1 \mathrm{~h}$. The solution was stirred a further $1 \mathrm{~h}$, then was washed with $1 \mathrm{M}$ aq. $\mathrm{HCl}$ and sat. aq. $\mathrm{NaHCO}_{3}$. The organic layer was dried over $\mathrm{MgSO}_{4}$, filtered and the solvent was evaporated under reduced pressure. The crude was subjected to flash chromatography $\left(\mathrm{SiO}_{2}, \mathrm{DCM}+1 \% \mathrm{MeOH}\right)$ to yield $4.57 \mathrm{~g}(73 \%)$ of the title compound $\mathbf{S 1 3}$ as pale yellow oil. ${ }^{1} \mathbf{H}-\mathbf{N M R}\left(300 \mathrm{MHz}, \mathrm{CDCl}_{3}\right): \delta=2.44\left(\mathrm{~s}, 6 \mathrm{H}, \mathrm{CH}_{3}\right), 3.58,3.61\left(2 \mathrm{~m}_{\mathrm{c}}\right.$, $16 \mathrm{H}), 3.68\left(\mathrm{~m}_{\mathrm{c}}, 4 \mathrm{H}, \mathrm{OCH}_{2} \mathrm{CH}_{2} \mathrm{OTs}\right), 4.15\left(\mathrm{~m}_{\mathrm{c}}, 4 \mathrm{H}, \mathrm{OCH}_{2} \mathrm{CH}_{2} \mathrm{OTs}\right), 7.34$ (bd, J = 8.3 Hz, $\left.4 \mathrm{H}, 3,5-\mathrm{H}_{\mathrm{ar}}\right), 7.79$ (bd, J = 8.3 Hz, 4 $\left.\mathrm{H}, 2,6-\mathrm{H}_{\mathrm{ar}}\right)$ ppm. ${ }^{13} \mathrm{C}$-NMR $\left(75 \mathrm{MHz}, \mathrm{CDCl}_{3}\right): \delta=21.8\left(\mathrm{CH}_{3}\right), 68.8\left(\mathrm{OCH}_{2} \mathrm{CH}_{2} \mathrm{OTs}\right), 69.4\left(\mathrm{OCH}_{2} \mathrm{CH}_{2} \mathrm{OTs}\right), 70.6,70.6,70.7,70.8$ $\left(\mathrm{CH}_{2}\right), 128.1$ (C-2,6), 129.9 (C-3,5), 133.0 (C-4), 144.9 (s, C-1) ppm. HRMS (pos. ESI-TOF): $m / z$ calculated for $\mathrm{C}_{26} \mathrm{H}_{39} \mathrm{O}_{11} \mathrm{~S}_{2}$ $[\mathrm{M}+\mathrm{H}]^{+} 591.1928$, found 591.1946 .

\section{1,17-Diazido-3,6,9,12,15-pentaoxaheptadecane (S14)}<smiles>CC(C)(CN)COCC(C)(C)CN</smiles>

Hexaethylene glycol ditosylate $(\mathbf{S 1 3}, 1.96 \mathrm{~g}, 3.32 \mathrm{mmol})$ was dissolved in acetone/water (22 ml, 2:1) and sodium azide (2.15 $\mathrm{g}, 33.2 \mathrm{mmol}$ ) was added. The solution was stirred at $80^{\circ} \mathrm{C}$ for $18 \mathrm{~h}$, then cooled down to r.t. and diluted with water and DCM. The organic phase was separated and the aq. phase was extracted three times with DCM. The combined org. phases were 
dried over $\mathrm{MgSO}_{4}$, filtered and the solvent was evaporated under reduced pressure to yield $1.10 \mathrm{~g}(98 \%)$ of the title compound S14 as colorless oil. ${ }^{1} \mathrm{H}-\mathrm{NMR}\left(300 \mathrm{MHz}, \mathrm{CDCl}_{3}\right): \delta=3.39$ (t, $\left.\mathrm{J}=5.1 \mathrm{~Hz}, 4 \mathrm{H}, \mathrm{CH}_{2} \mathrm{~N}_{3}\right), 3.64-3.70\left(\mathrm{~m}, 20 \mathrm{H}, \mathrm{CH}_{2}\right) \mathrm{ppm} .{ }^{13} \mathbf{C}-\mathrm{NMR}$ $\left(75 \mathrm{MHz}, \mathrm{CDCl}_{3}\right): \delta=50.8\left(\mathrm{CH}_{2} \mathrm{~N}_{3}\right), 70.2,70.7,70.8,70.8,70.8\left(\mathrm{CH}_{2}\right) \mathrm{ppm}$.

\section{7-Azido-3,6,9,12,15-pentaoxaheptadecan-1-amine (S15)}<smiles>CC(C)(CN)COCC(C)(C)CN</smiles>

1,17-Diazido-3,6,9,12,15-pentaoxaheptadecane (S14, $2.44 \mathrm{~g}, 7.34 \mathrm{mmol})$ was dissolved in toluene/ethanol/5\% aq. $\mathrm{HCl}$ $(10 / 3 / 15 \mathrm{ml})$ and triphenyl phosphine $(2.02 \mathrm{~g}, 7.71 \mathrm{mmol})$ dissolved in toluene $(30 \mathrm{ml})$ was added dropwise during the course of $1 \mathrm{~h}$. The mixture was stirred for $72 \mathrm{~h}$ at r.t., then $2 \mathrm{M} \mathrm{HCl}$ was added and the aq. phase was separated. The aqueous layer was extracted twice with diethyl ether and subsequently the $\mathrm{pH}$ was adjusted to 14 by addition of $2 \mathrm{M} \mathrm{NaOH}$. The aq. phase was extracted twice with DCM and the combined org. layers were dried over MgSO4, filtered and evaporated under reduced pressure to furnish $2.0 \mathrm{~g}(89 \%)$ of the title compound $\mathbf{S 1 5}$ as colorless oil. ${ }^{1} \mathbf{H}-\mathbf{N M R}\left(300 \mathrm{MHz}, \mathrm{CDCl}_{3}\right): \delta=2.87(\mathrm{t}, \mathrm{J}=5.0 \mathrm{~Hz}$, $\left.2 \mathrm{H}, \mathrm{NH}_{2}\right), 3.40$ (t, $\left.J=5.0 \mathrm{~Hz}, 2 \mathrm{H}, \mathrm{CH}_{2} \mathrm{NH}_{2}\right), 3.52\left(\mathrm{t}, J=5.0 \mathrm{~Hz}, 2 \mathrm{H}, \mathrm{CH}_{2} \mathrm{CH}_{2} \mathrm{NH}_{2}\right), 3.61-3.72\left(\mathrm{~m}, 20 \mathrm{H}, \mathrm{CH}_{2}\right) \mathrm{ppm} .{ }^{13} \mathrm{C}-\mathrm{NMR}$ $\left(75 \mathrm{MHz}, \mathrm{CDCl}_{3}\right): \delta=41.9\left(\mathrm{CH}_{2} \mathrm{NH}_{2}\right), 50.8\left(\mathrm{CH}_{2} \mathrm{~N}_{3}\right), 70.1,70.4,70.7,70.7,70.7,70.8,70.8\left(\mathrm{CH}_{2}\right), 73.6\left(C_{2} \mathrm{CH}_{2} \mathrm{NH}_{2}\right) \mathrm{ppm}$ HRMS (pos. ESI-TOF): $\mathrm{m} / z$ calculated for $\mathrm{C}_{12} \mathrm{H}_{27} \mathrm{~N}_{4} \mathrm{O}_{5}[\mathrm{M}+\mathrm{H}]^{+}$307.1976, found 307.1979.

tert-Butyl (17-azido-3,6,9,12,15-pentaoxaheptadecyl)carbamate (S16)<smiles>CC(C)(C)CCCNCC(C)(C)COCC(C)(C)CN</smiles>

17-Azido-3,6,9,12,15-pentaoxaheptadecan-1-amine (S15, $1.16 \mathrm{~g}, 3.79 \mathrm{mmol})$ was dissolved in 1,4-dioxane (5 ml) and sat. aq. $\mathrm{NaHCO}_{3}(10 \mathrm{ml})$ was added. Boc anhydride $(1.09 \mathrm{~g}, 5.0 \mathrm{mmol})$ dissolved in 1,4-dioxane $(10 \mathrm{ml})$ was added. The mixture was stirred at r.t. for $4 \mathrm{~h}$. It was then diluted with $\mathrm{DCM}$, washed with water, dried over $\mathrm{MgSO}_{4}$, and the solvent was evaporated under reduced pressure. Purification of the crude by flash chromatography $\left(\mathrm{SiO}_{2}, \mathrm{DCM}+3 \% \mathrm{MeOH}\right)$ yielded $1.26 \mathrm{~g} \mathrm{(82 \% )} \mathrm{of}$ the title compound $\mathbf{S 1 6}$ as colorless oil. ${ }^{1} \mathrm{H}-\mathrm{NMR}\left(300 \mathrm{MHz}, \mathrm{CDCl}_{3}\right): \delta=1.44\left(\mathrm{~s}, 9 \mathrm{H}, \mathrm{C}\left(\mathrm{CH}_{3}\right)_{3}\right), 3.31(\mathrm{q}, J=5.3 \mathrm{~Hz}, 2 \mathrm{H}$, $\mathrm{CONHCH}_{2}$ ), $3.39\left(\mathrm{t}, J=5.1 \mathrm{~Hz}, 2 \mathrm{H}, \mathrm{N}_{3} \mathrm{CH}_{2} \mathrm{CH}_{2}\right), 3.53\left(\mathrm{t}, J=5.1 \mathrm{~Hz}, 2 \mathrm{H}, \mathrm{N}_{3} \mathrm{CH}_{2} \mathrm{CH}_{2}\right), 3.58-3.72\left(\mathrm{~m}, 18 \mathrm{H}, \mathrm{CH}_{2}\right), 5.03(\mathrm{bs}, 1 \mathrm{H}$, $\mathrm{NHCO}) \mathrm{ppm}$.

\section{tert-Butyl (17-amino-3,6,9,12,15-pentaoxaheptadecyl)carbamate (S17)}<smiles>CC(C)(C)CNCC(C)(C)COCC(C)(C)CN</smiles>

tert-Butyl (17-azido-3,6,9,12,15-pentaoxaheptadecyl)carbamate (S16, $726 \mathrm{mg}, 1.79 \mathrm{mmol}$ ) was dissolved in THF and triphenyl phosphine $(620 \mathrm{mg}, 2.36 \mathrm{mmol})$ and water $(100 \mu \mathrm{l}, 5.55 \mathrm{mmol})$ were added. The solution was heated to $60^{\circ} \mathrm{C}$ for $18 \mathrm{~h} .1 \mathrm{M}$ $\mathrm{HCl}$ was added and the solution was washed with toluene $(3 \mathrm{x})$. The aq. phase was adjusted to basic $\mathrm{pH}$ with $1 \mathrm{M} \mathrm{NaOH}$ and extracted with DCM (3x). The combined org. phases were dried over $\mathrm{MgSO}_{4}$ and the solvents were evaporated under reduced pressure to yield $670 \mathrm{mg}(70 \%)$ of the title compound $\mathbf{S 1 7}$ as light yellow oil. ${ }^{1} \mathbf{H}-\mathbf{N M R}\left(300 \mathrm{MHz}, \mathrm{CDCl}_{3}\right): \delta=1.42(\mathrm{~s}, 9 \mathrm{H}$, $\left.\mathrm{C}\left(\mathrm{CH}_{3}\right)\right), 1.81$ (bs, $2 \mathrm{H}, \mathrm{NH}_{2}$ ), 2.85 (t, J = 5.2 Hz, $2 \mathrm{H}, \mathrm{CH}_{2} \mathrm{NH}_{2}$ ), 3.29 (q, J = 5.4 Hz, $\left.2 \mathrm{H}, \mathrm{CH}_{2} \mathrm{NHBoc}\right), 3.50$ (t, J = $5.2 \mathrm{~Hz}, 2 \mathrm{H}$, $\mathrm{CH}_{2} \mathrm{CH}_{2} \mathrm{NH}_{2}$ ), 3.52 (t, $\mathrm{J}=5.4 \mathrm{~Hz}, 2 \mathrm{H}, \mathrm{CH}_{2} \mathrm{CH}_{2} \mathrm{NHBoc}$ ), 3.56-3.68 (m, $\left.16 \mathrm{H}, \mathrm{CH}_{2}\right), 5.03-5.32$ (m, $\left.1 \mathrm{H}, \mathrm{NHBoc}\right)$ ppm. ${ }^{13} \mathrm{C}-\mathrm{NMR}$ $\left(75 \mathrm{MHz}, \mathrm{CDCl}_{3}\right): \delta=28.5\left(\mathrm{C}\left(\mathrm{CH}_{3}\right)_{3}\right), 40.5\left(\mathrm{CH}_{2} \mathrm{NHBoc}\right), 41.9\left(\mathrm{CH}_{2} \mathrm{NH}_{2}\right), 70.3,70.4,70.6,70.7,70.7,70.7\left(\mathrm{CH}_{2}\right), 73.4$

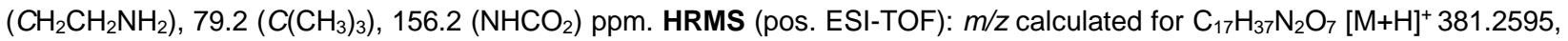
found 381.2605 . 
Synthesis of DOTA $\mathbf{N - 1 , 7 - b i s ( b e n z y l ~ e s t e r ) ^ { 7 }}$
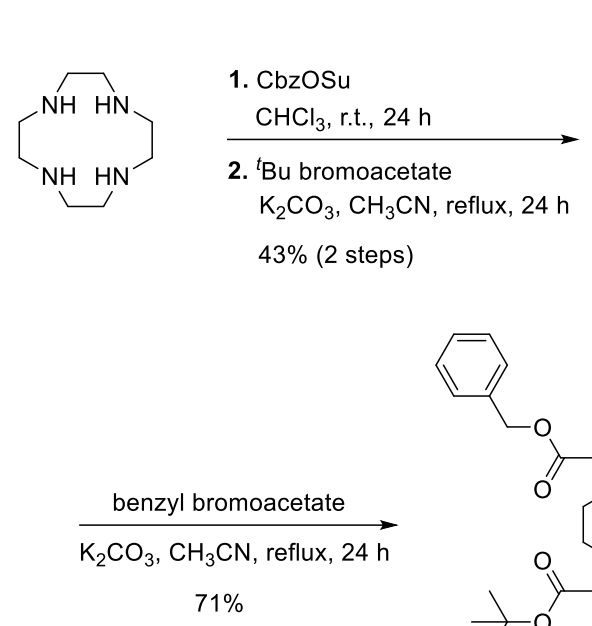

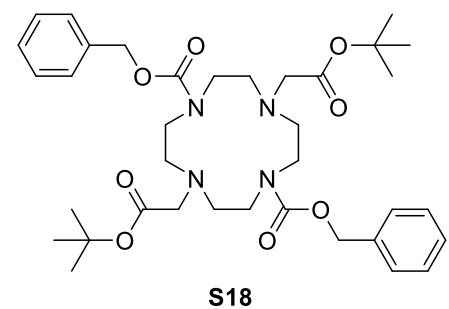

S18

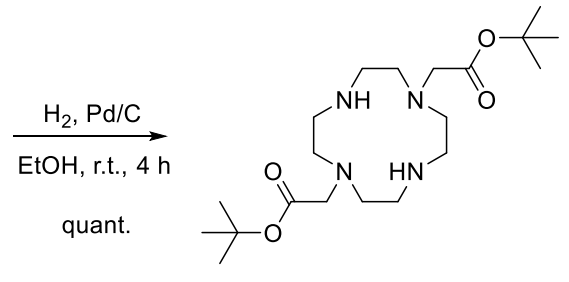

S19
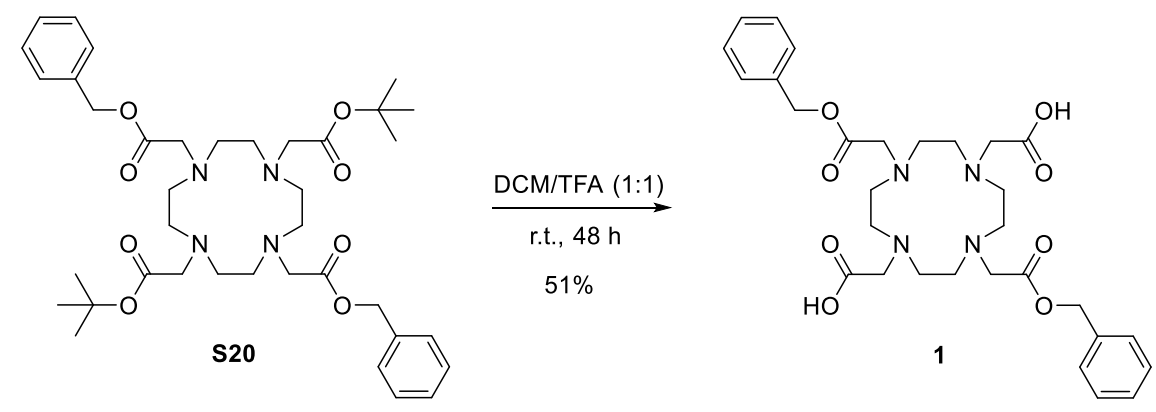

Dibenzyl 4,10-bis(2-(tert-butoxy)-2-oxoethyl)-1,4,7,10-tetraazacyclododecane-1,7-dicarboxylate (S18)<smiles>CN(CCNCC(=O)OC(C)(C)C)CCN(CCNCC(=O)OC(C)(C)C)CC(=O)O</smiles>

1,4,7,10-Tetraazacyclododecane $(2.00 \mathrm{~g}, 11.6 \mathrm{mmol})$ was dissolved in chloroform $(50 \mathrm{ml})$ and $\mathrm{N}$ (benzyloxycarbonyloxy)succinimide $(5.79 \mathrm{~g}, 23.2 \mathrm{mmol})$ was added in one portion and the solution was stirred for $24 \mathrm{~h}$ at r.t. The solvent was evaporated under reduced pressure and the residue was resuspended in aq. $1 \mathrm{M} \mathrm{NaOH}$ and extracted with DCM. The combined organic phases were dried over $\mathrm{MgSO}_{4}$ and the solvent was evaporated under reduced pressure to yield $5.11 \mathrm{~g}$ colorless oil which was used without further purification. The oil was dissolved in acetonitrile $(50 \mathrm{ml})$ and freshly ground potassium carbonate $(1.48 \mathrm{~g}, 10.7 \mathrm{mmol})$ and tert-butyl bromoacetate $(1.26 \mathrm{ml}, 8.58 \mathrm{mmol})$ were added and the mixture was refluxed for $18 \mathrm{~h}$. It was then allowed to cool down to r.t., filtered over a pad of celite, and the solvent was evaporated under reduced pressure. Purification of the residue by flash chromatography (basic $\left.\mathrm{Al}_{2} \mathrm{O}_{3}, \mathrm{cHex} / \mathrm{EtOAc}=91: 9\right)$ yielded $2.44 \mathrm{~g}(43 \%)$ of the title compound $\mathbf{S 1 8}$ as colorless oil. ${ }^{1} \mathrm{H}-\mathrm{NMR}\left(300 \mathrm{MHz}, \mathrm{CDCl}_{3}\right): \delta=1.42(\mathrm{~s}, 18 \mathrm{H}, \mathrm{tBu}), 2.87\left(\mathrm{bs}, 8 \mathrm{H}, \mathrm{CH}_{2}\right), 3.15-3.50$ (m, $\left.12 \mathrm{H}, \mathrm{CH}_{2}, \mathrm{NCH}_{2} \mathrm{CO}_{2}\right), 5.12\left(\mathrm{~s}, 4 \mathrm{H}, \mathrm{CH}_{2} \mathrm{Ar}\right), 7.27-7.38$ (m, $\left.10 \mathrm{H}, \mathrm{Ar}\right)$ ppm. ${ }^{13} \mathrm{C}-\mathrm{NMR}\left(75 \mathrm{MHz}, \mathrm{CDCl}_{3}\right): \delta=28.3(\mathrm{C}(\mathrm{CH}) 3$ ), $47.0\left(\mathrm{CH}_{2} \mathrm{NCO}_{2}\right), 54.3\left(\mathrm{CH}_{2} \mathrm{NCH}_{2} \mathrm{CO}_{2}\right), 56.1\left(\mathrm{NCH}_{2} \mathrm{CO}_{2}\right), 67.1\left(\mathrm{CH}_{2} \mathrm{Ar}\right), 81.1\left(\mathrm{C}_{\left(\mathrm{CH}_{3}\right)}\right), 128.0,128.1,128.6(\mathrm{Ar}), 137.0\left(\mathrm{CH}_{2} \mathrm{C}_{\mathrm{Ar}}\right)$, $156.6\left(\mathrm{NCO}_{2}\right), 167.9\left(\mathrm{CO}_{2}\right.$ tBu$)$ ppm. HRMS (pos. ESI-TOF): $\mathrm{m} / \mathrm{z}$ calculated for $\mathrm{C}_{36} \mathrm{H}_{53} \mathrm{~N}_{4} \mathrm{O}_{8}[\mathrm{M}+\mathrm{H}]^{+} 669.3858$, found 669.3867

\section{Di-tert-butyl 2,2'-(1,4,7,10-tetraazacyclododecane-1,7-diyl)diacetate (S19)}<smiles>CC(C)(C)OC(=O)CN1CCNCCN(CC(=O)OC(C)(C)C)CCNCC1</smiles>

Dibenzyl 4,10-bis(2-(tert-butoxy)-2-oxoethyl)-1,4,7,10-tetraazacyclododecane-1,7-dicarboxy-late (S18, $2.44 \mathrm{~g}, 3.65 \mathrm{mmol}$ ) was dissolved in $\mathrm{EtOH}(30 \mathrm{ml})$ and $10 \%$ palladium on activated charcoal was added. A balloon filled with hydrogen gas was installed on the flask which was allowed to bubble through the solution for $3 \mathrm{~h}$ at r.t. The mixture was filtered over a pad of celite and the solvent was evaporated under reduced pressure to afford $1.46 \mathrm{~g}$ (quant.) of the title compound $\mathbf{S} 19$ as colorless solid. ${ }^{1} \mathbf{H}$ - 
NMR (300 MHz, DMSO-D $): \delta=1.44(\mathrm{~s}, 18 \mathrm{H}, \mathrm{tBu}), 2.94$ (bs, $\left.8 \mathrm{H}, \mathrm{CH}_{2}\right), 3.06$ (bs, $\left.8 \mathrm{H}, \mathrm{CH}_{2}\right), 3.43\left(\mathrm{~s}, 4 \mathrm{H}_{1} \mathrm{CH}_{2} \mathrm{CO}_{2}\right) \mathrm{ppm} .{ }^{13} \mathrm{C}-$ NMR $(75 \mathrm{MHz}$, DMSO-D $): \delta=27.9\left(\mathrm{C}(\mathrm{CH})_{3}\right), 42.5\left(\mathrm{CH}_{2}\right), 48.4\left(\mathrm{CH}_{2}\right), 54.3\left(\mathrm{CH}_{2} \mathrm{CO}_{2}\right), 81.2\left(C\left(\mathrm{CH}_{3}\right)_{2}\right), 170.6\left(\mathrm{CO}_{2}\right)$ ppm. HRMS (pos. ESI-TOF): $m / z$ calculated for $\mathrm{C}_{20} \mathrm{H}_{41} \mathrm{~N}_{4} \mathrm{O}_{4}[\mathrm{M}+\mathrm{H}]^{+} 401.3122$, found 401.3124 .

\section{DOTA $\mathbf{N - 1 , 7 - b i s ( b e n z y l ) - N - 4 , 1 0 - b i s ( t e r t - b u t y l ~ e s t e r ) ~ ( S 2 0 ) ~}$}

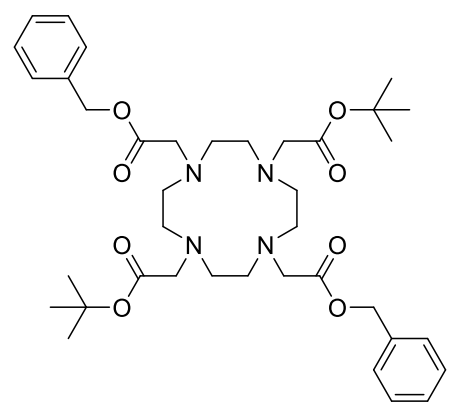

Di-tert-butyl 2,2'-(1,4,7,10-tetraazacyclododecane-1,7-diyl)diacetate (S19, $1.46 \mathrm{~g}, 3.65 \mathrm{mmol})$ was dissolved in acetonitrile (50 $\mathrm{ml}$ ) and freshly ground potassium carbonate $(1.26 \mathrm{~g}, 9.12 \mathrm{mmol})$ and benzyl bromoacetate $(1.07 \mathrm{ml}, 6.75 \mathrm{mmol})$ were added. The mixture was refluxed for $24 \mathrm{~h}$, filtered over a pad of celite and the solvent was evaporated under reduced pressure. Flash chromatography (basic $\mathrm{Al}_{2} \mathrm{O}_{3}, \mathrm{DCM}, 0-3 \% \mathrm{MeOH}$ ) afforded $1.80 \mathrm{~g}(71 \%)$ of the title compound $\mathbf{S} 20$ as pale yellow oil. ${ }^{1} \mathbf{H}-\mathbf{N M R}$ $\left(300 \mathrm{MHz}, \mathrm{CDCl}_{3}\right): \delta=1.42(\mathrm{~s}, 18 \mathrm{H}, t \mathrm{Bu}), 2.50-3.57\left(\mathrm{~m}, 24 \mathrm{H}, \mathrm{CH}_{2}\right), 5.05-5.18\left(\mathrm{~m}, 4 \mathrm{H}, \mathrm{CH}_{2} \mathrm{Ar}\right), 7.27-7.40(\mathrm{~m}, 10 \mathrm{H}, \mathrm{Ar}) \mathrm{ppm}$. ${ }^{13}$ C-NMR $\left(75 \mathrm{MHz}, \mathrm{CDCl}_{3}\right): \delta=28.3\left(\mathrm{C}\left(\mathrm{CH}_{3}\right)_{3}\right), 52.0,52.1\left(\mathrm{NCH}_{2} \mathrm{CH}_{2}\right), 55.7,56.7\left(\mathrm{CH}_{2} \mathrm{CO}_{2}\right), 66.0\left(\mathrm{CH}_{2} \mathrm{Ar}\right), 80.8\left(C\left(\mathrm{CH}_{3}\right)_{3}\right)$, 127.1, 128.4, $128.7(\mathrm{Ar}), 136.0\left(\mathrm{CH}_{2} \mathrm{C}_{\mathrm{Ar}}\right), 171.1,171.7\left(\mathrm{CO}_{2} \mathrm{tBu}, \mathrm{CO}_{2} \mathrm{Bn}\right)$ ppm. HRMS (pos. ESI-TOF): $\mathrm{m} / \mathrm{z}$ calculated for $\mathrm{C}_{38} \mathrm{H}_{57} \mathrm{~N}_{4} \mathrm{O}_{8}[\mathrm{M}+\mathrm{H}]^{+}$697.4171, found 697.4183 .

\section{DOTA N-1,7-bis(benzyl ester) (1)}

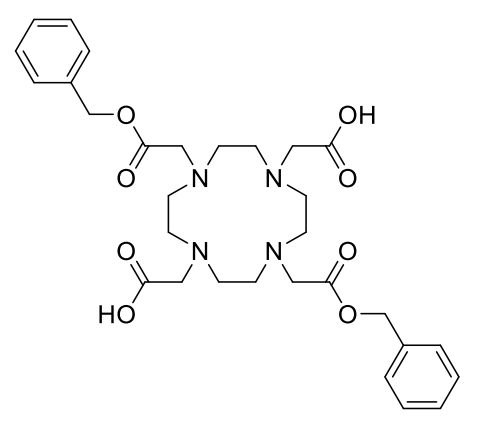

DOTA $N$-1,7-bisbenzyl N-4,10-bis(tert-butyl) ester (S20, $514 \mathrm{mg}, 0.74 \mathrm{mmol}$ ) was dissolved in DCM/TFA (15 ml, 1:1) and the solution was stirred at r.t. for $48 \mathrm{~h}$. The solvent was coevaporated with toluene and the residue was purified by HPLC (5-75\% ACN in $20 \mathrm{~min}, 75-100 \%$ in $2 \mathrm{~min}$, then $3 \mathrm{~min} 100 \%)$ to yield $219 \mathrm{mg} \mathrm{(51 \% )} \mathrm{of} \mathrm{the} \mathrm{title} \mathrm{compound} 1$ as colorless powder after lyophilization. ${ }^{1} \mathrm{H}-\mathrm{NMR}\left(300 \mathrm{MHz}, \mathrm{CD}_{3} \mathrm{CN}\right): \delta=3.00\left(\mathrm{~m}_{\mathrm{c}}, 8 \mathrm{H}, \mathrm{CH}_{2}\right), 3.33\left(\mathrm{~m}_{\mathrm{c}}, 8 \mathrm{H}, \mathrm{CH}_{2}\right), 3.64,3.99\left(2 \mathrm{~s}, 8 \mathrm{H}, \mathrm{CH}_{2} \mathrm{CO}_{2}\right), 5.09$ (s, $\left.4 \mathrm{H}, \mathrm{CH}_{2} \mathrm{Ar}\right), 7.27-7.40(\mathrm{~m}, 10 \mathrm{H}, \mathrm{Ar})$ ppm. ${ }^{13} \mathrm{C}-\mathrm{NMR}\left(75 \mathrm{MHz}, \mathrm{CD}_{3} \mathrm{CN}\right): \delta=49.1\left(\mathrm{CH}_{2}\right), 52.5\left(\mathrm{CH}_{2}\right), 53.8\left(\mathrm{CH}_{2} \mathrm{CO}_{2}\right), 55.8$ $\left(\mathrm{CH}_{2} \mathrm{CO}_{2}\right), 67.7\left(\mathrm{CH}_{2} \mathrm{Ar}\right), 129.3,129.3,129.5(\mathrm{Ar}), 136.6(\mathrm{Ar}), 168.8,172.2\left(\mathrm{CO}_{2}\right)$ ppm. HRMS (pos. ESI-TOF): $\mathrm{m} / z$ calculated for $\mathrm{C}_{30} \mathrm{H}_{41} \mathrm{~N}_{4} \mathrm{O}_{8}[\mathrm{M}+\mathrm{H}]^{+}$585.2919, found 585.2926. 
<smiles>O=C(O)CN(CCN(CCN(CC(=O)O)CC(=O)O)CC(=O)O)CCN(CC(=O)O)CC(=O)O</smiles>

1
1. BocNHPEG $\mathrm{NH}_{2}, \mathrm{HATU}$, DIPEA, $\mathrm{CH}_{3} \mathrm{CN}$, r.t., $4 \mathrm{~h}$

2. $\mathrm{H}_{2}, \mathrm{Pd} / \mathrm{C}, \mathrm{EtOH}$, r.t., $4 \mathrm{~h}$

$72 \%$

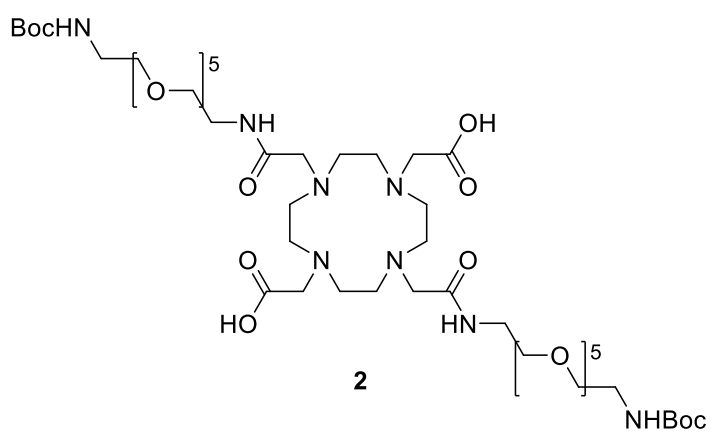

\section{Bis-(PEGNHBoc)-dibenzyl DOTAM (S21)}

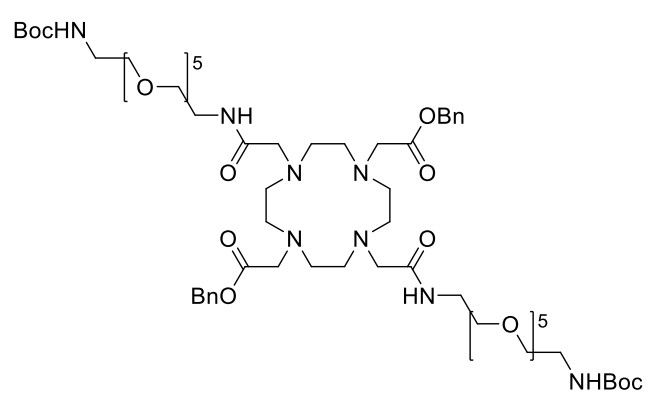

DOTA $N$-1,7-bis(benzyl ester) $(1,100 \mathrm{mg}, 0.17 \mathrm{mmol})$ was dissolved in acetonitrile $(10 \mathrm{ml})$ and DIPEA (119 $\mu \mathrm{l}, 0.68 \mathrm{mmol})$ and HATU (149 mg, $0.39 \mathrm{mmol}$ ) were added. The mixture was stirred for $10 \mathrm{~min}$ at r.t., then tert-butyl (17-amino-3,6,9,12,15pentaoxaheptadecyl)carbamate $(\mathbf{S 1 7}, 176 \mathrm{mg}, 0.46 \mathrm{mmol})$ was added and the reaction was stirred for $4 \mathrm{~h}$ at $\mathrm{r} . \mathrm{t}$. The solvent was evaporated under reduced pressure and the residue was purified by HPLC (5-75\% ACN in 22 min). The fractions containing product were combined, diluted with $\mathrm{DCM}$, washed with sat. aq. $\mathrm{NaHCO}_{3}$ and dried over $\mathrm{MgSO}_{4}$. Evaporation of the solvents yielded $168 \mathrm{mg}(75 \%)$ of the title compound $\mathbf{S 2 1}$ as colorless oil. ${ }^{1} \mathbf{H}-\mathbf{N M R}\left(300 \mathrm{MHz}, \mathrm{CDCl}_{3}\right): \delta=1.42,1.44(2 \mathrm{~s}$, $\left.18 \mathrm{H}, \mathrm{C}\left(\mathrm{CH}_{3}\right)_{3}\right), 2.20-4.07(\mathrm{~m}, 72 \mathrm{H}), 5.08\left(\mathrm{~s}, 4 \mathrm{H}, \mathrm{CH}_{2} \mathrm{Ar}\right), 7.20-7.40(\mathrm{~m}, 10 \mathrm{H}, \mathrm{Ar}) \mathrm{ppm} .{ }^{13} \mathrm{C}-\mathrm{NMR}\left(75 \mathrm{MHz} \mathrm{CDCl}_{3}\right): \delta=28.6$ $\left(\mathrm{C}\left(\mathrm{CH}_{3}\right)_{3}\right)$, 39.7, $40.5\left(\mathrm{CONHCH} \mathrm{CH}_{2} \mathrm{O}, \mathrm{CH}_{2} \mathrm{NHBoc}\right), 48.2,52.0\left(\mathrm{NCH}_{2} \mathrm{CH}_{2} \mathrm{~N}\right)$, 53.2, $55.3\left(\mathrm{NCH}_{2} \mathrm{CO}\right), 66.9\left(\mathrm{CH}_{2} \mathrm{Ar}\right), 69.1,70.2$,

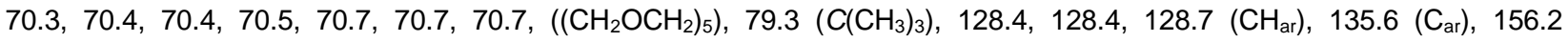
(NHCOOtBu), 171.4, $175.9\left(\mathrm{CO}_{2} \mathrm{Bn}, \mathrm{CONH}\right)$ ppm. HRMS (pos. ESI-TOF): $m / z$ calculated for $\mathrm{C}_{64} \mathrm{H}_{109} \mathrm{~N}_{8} \mathrm{O}_{20}[\mathrm{M}+\mathrm{H}]^{+} 1309.7753$, found 1309.7808 .

\section{Bis-(PEGNHBoc) DOTAM (2)}

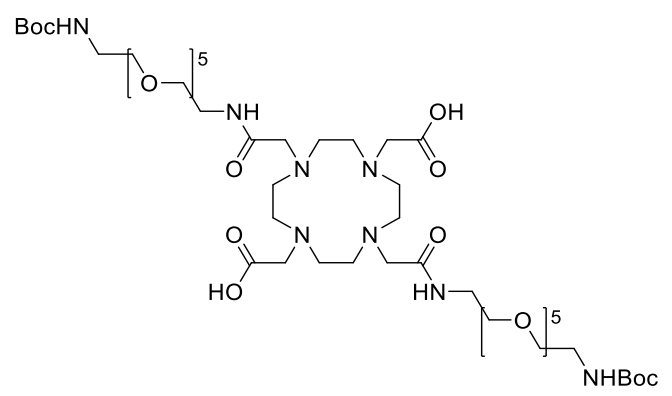

Bis-(PEGNHBoc)-dibenzyl DOTA (S21, $225 \mathrm{mg}, 0.17 \mathrm{mmol}$ ) was dissolved in $\mathrm{EtOH}(15 \mathrm{ml}$ ) and 10\% palladium on activated charcoal was added. A balloon filled with hydrogen gas was installed on the flask which was allowed to bubble through the solution for $3 \mathrm{~h}$ at r.t. The mixture was filtered over a pad of celite and the solvent was evaporated under reduced pressure to afford $186 \mathrm{mg}(96 \%)$ of the title compound 2 as colorless oil. ${ }^{1} \mathrm{H}-\mathrm{NMR}\left(300 \mathrm{MHz}, \mathrm{CD}_{3} \mathrm{CN}\right): \delta=1.40\left(\mathrm{~s}, 3 \mathrm{H},\left(\mathrm{C}\left(\mathrm{CH}_{3}\right)_{3}\right)\right), 2.98-$ $5.02(\mathrm{~m}, 72 \mathrm{H}), 5.47\left(\mathrm{~s}, 2 \mathrm{H}, \mathrm{NH}_{\mathrm{Boc}}\right), 7.27(\mathrm{~s}, 2 \mathrm{H}, \mathrm{CONH})$ ppm. ${ }^{13} \mathrm{C}-\mathrm{NMR}\left(75 \mathrm{MHz}, \mathrm{CD}_{3} \mathrm{CN}\right): \delta=28.5,28.5\left(\mathrm{C}\left(\mathrm{CH}_{3}\right)_{3}\right), 40.1$, $40.9\left(\mathrm{CONHCH} \mathrm{CH}_{2} \mathrm{O}, \mathrm{CH}_{2} \mathrm{NHBoc}\right)$, 49.7, $51.6\left(\mathrm{NCH}_{2} \mathrm{CH}_{2} \mathrm{~N}\right)$, 54.0, 56.1 $\left(\mathrm{NCH}_{2} \mathrm{CO}\right)$, 69.7, 70.5, 70.6, 70.7, 70.8, 70.9, 70.9, 


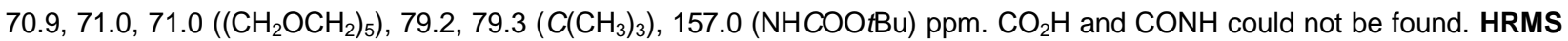
(pos. ESI-TOF): $m / z$ calculated for $\mathrm{C}_{50} \mathrm{H}_{97} \mathrm{~N}_{8} \mathrm{O}_{20}[\mathrm{M}+\mathrm{H}]^{+} 1129.6814$, found 1129.6826 .

\section{Bis-(PEGNHBoc)-Cy3 DOTAM (3)}

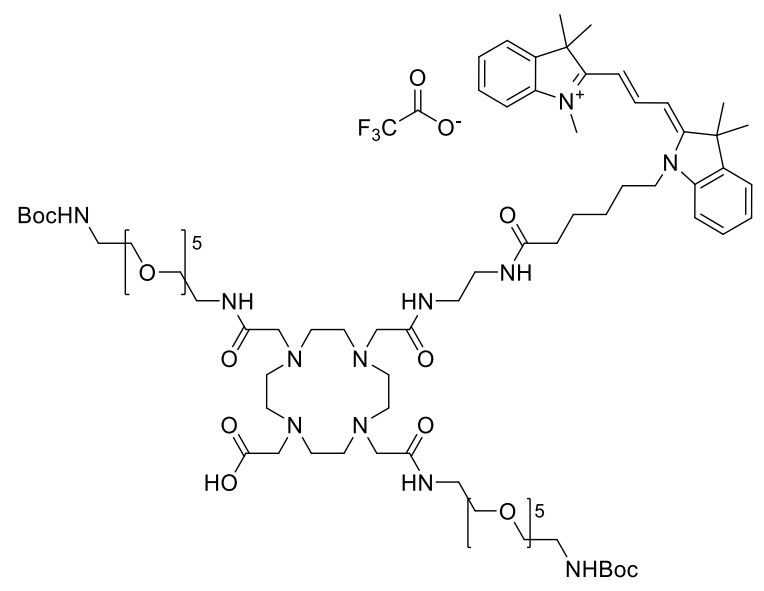

Bis-(PEGNHBoc) DOTAM (2, $32 \mathrm{mg}, 28.3 \mu \mathrm{mol})$ was dissolved in acetonitrile $(5 \mathrm{ml})$ and DIPEA $(20 \mu \mathrm{l}, 113 \mu \mathrm{mol})$ and HATU (10 mg, $25 \mu \mathrm{mol}$ ) were added. The solution was stirred for $10 \mathrm{~min}$, then Cy3-EDA (S8, $19 \mathrm{mg}, 26 \mu \mathrm{mol})$ was added and the reaction was stirred for $4 \mathrm{~h}$. The crude solution was purified by HPLC (5-75\% ACN in 22 min, 75-100\% in 4 min, no TFA) to yield $12 \mathrm{mg} \mathrm{(29 \% )} \mathrm{Bis-(PEGNHBoc)-Cy3} \mathrm{DOTAM} \mathrm{(3)} \mathrm{as} \mathrm{pink} \mathrm{powder} \mathrm{after} \mathrm{lyophilization.}{ }^{1} \mathrm{H}-\mathrm{NMR}\left(300 \mathrm{MHz}, \mathrm{CD}_{3} \mathrm{CN}\right): \delta=1.39$ $\left(\mathrm{s}, 18 \mathrm{H},\left(\mathrm{C}\left(\mathrm{CH}_{3}\right)_{3}\right)\right), 1.46\left(\mathrm{~m}, 2 \mathrm{H}, \mathrm{CH}_{2, \mathrm{Cy}}\right), 1.63\left(\mathrm{~m}, 2 \mathrm{H}, \mathrm{CH}_{2, \mathrm{Cy}}\right), 1.71\left(\mathrm{~s}, 12 \mathrm{H}, \mathrm{C}\left(\mathrm{CH}_{3}\right)_{2}\right), 1.77\left(\mathrm{~m}, 2 \mathrm{H}, \mathrm{CH}_{2, \mathrm{Cy}}\right), 2.16(\mathrm{~m}, 2$ $\left.\mathrm{H}, \mathrm{CH}_{2} \mathrm{CO}_{\mathrm{Cy} 3}\right), 3.59\left(\mathrm{NCH}_{3}\right), 4.03\left(\mathrm{t}, \mathrm{J}=7.7 \mathrm{~Hz}, 2 \mathrm{H}, \mathrm{NCH}_{2, \mathrm{Cy}}\right), 3.04-3.69\left(\mathrm{~m}, 76 \mathrm{H}, \mathrm{CH}_{2}\right), 5.46\left(\mathrm{~s}, 2 \mathrm{H}, \mathrm{NH}_{\mathrm{Boc}}\right), 6.34,6.35(2 \mathrm{~d}$, $J=13.5 \mathrm{~Hz}, 2 \mathrm{H}, \mathrm{a}, \mathrm{c}-\mathrm{H}), 7.26-7.55\left(\mathrm{~m}, 8 \mathrm{H}, \mathrm{CH}_{\mathrm{ar}}\right), 8.45(\mathrm{t}, J=13.5 \mathrm{~Hz}, 1 \mathrm{H}, \mathrm{b}-\mathrm{H}) \mathrm{ppm} .{ }^{13} \mathrm{C}-\mathrm{NMR}\left(75 \mathrm{MHz}, \mathrm{CD}_{3} \mathrm{CN}\right): \delta=26.0$, 27.1 , 27.7, 28.0, 28.2, 28.6, 32.1, 36.5, 39.5, 39.9, 40.0, 40.9, 45.1, 50.2, 50.2, 51.3, 51.7, 56.3, 69.9, 70.5, 70.8, 70.8, 71.0, 71.0, 71.0, 79.2, 103.2, 103.4, 112.1, 112.2, 123.2, 123.3, 126.3, 129.6, 129.7, 141.8, 141.9, 143.1, 143.9, 151.4, 156.8, 160.5, 161.0, 173.4, 174.1, 174.5, 175.4, 176.0, 178.5 ppm. HRMS (pos. ESI-TOF): $m / z$ calculated for $\mathrm{C}_{82} \mathrm{H}_{137} \mathrm{~N}_{12} \mathrm{O}_{20}[\mathrm{M}]{ }^{+} 1610.0067$, found 1610.0075 .

\section{Bis-(PEGNH2)-Cy3-CatSseq-BHQ-2 DOTAM (4)}

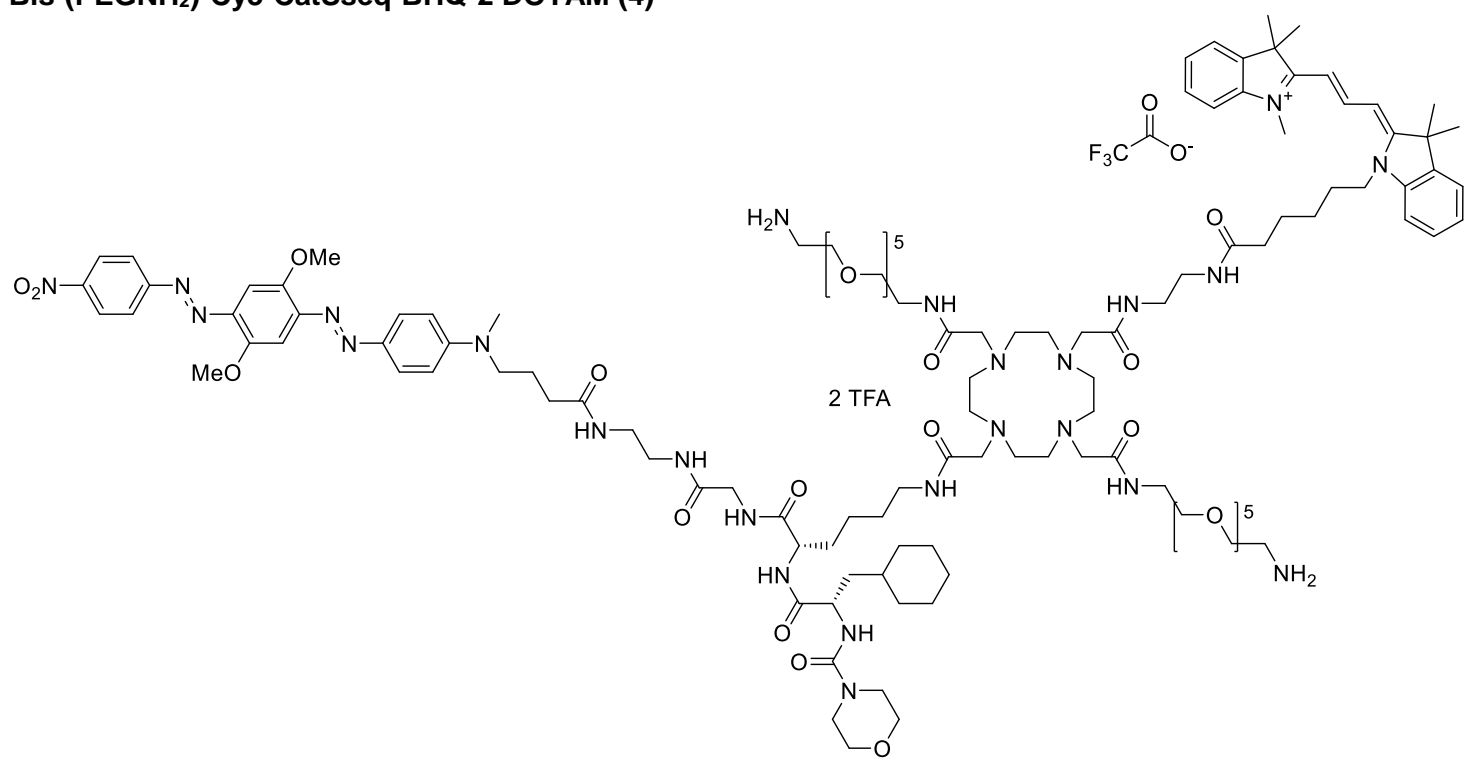

Bis-(PEGNHBoc)-Cy3 DOTAM (3, $22 \mathrm{mg}, 13.6 \mu \mathrm{mol})$ was dissolved in acetonitrile $(5 \mathrm{ml})$ and DIPEA (12 $\mu \mathrm{l}, 68 \mu \mathrm{mol})$ and HATU $(5 \mathrm{mg}, 13.6 \mu \mathrm{mol})$ were added. The solution was stirred for $10 \mathrm{~min}$, then CatS-sequence-BHQ-2 (16 mg, $14.3 \mu \mathrm{mol})$ was added and the reaction was stirred for $3 \mathrm{~h}$. The solvents were evaporated under reduced pressure and TFA/DCM (1:1, $5 \mathrm{ml})$ was added and the solution was stirred for $1 \mathrm{~h}$ at r.t. The solvents were evaporated under reduced pressure and the crude was purified by HPLC (5-75\% ACN in $22 \mathrm{~min}, 75-100 \%$ in $4 \mathrm{~min})$ to yield $15 \mathrm{mg}$ (44\%) Bis-(PEGNH $)_{2}$-Cy3-CatSseq-BHQ-2 DOTAM 
(4) as purple powder after lyophilization. HRMS (pos. ESI-TOF): $m / z$ calculated for $\mathrm{C}_{121} \mathrm{H}_{188} \mathrm{~N}_{25} \mathrm{O}_{25}[\mathrm{M}+2 \mathrm{H}]^{3+} 798.1461$, found 798.1485 .

\section{Cy3/BHQ-2 CatS activatable control probe (6)}

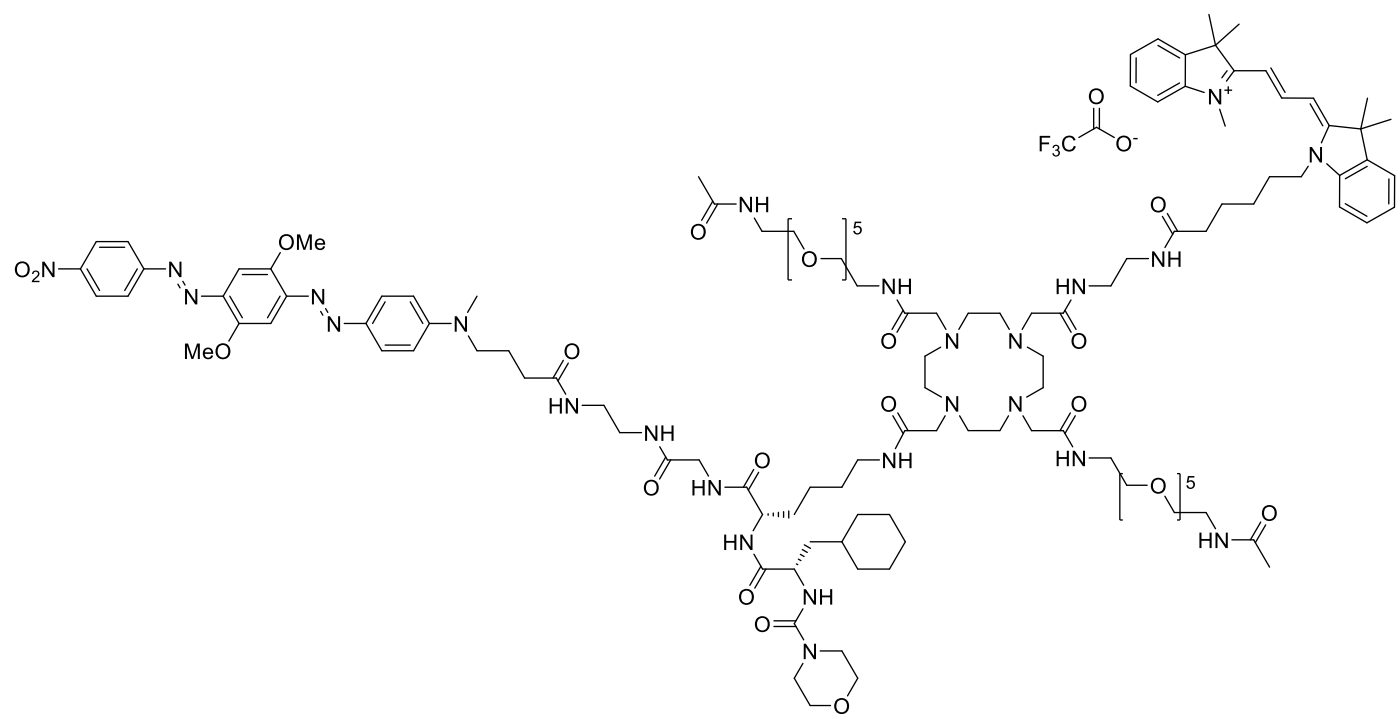

Bis-(PEGNH $)_{2}$-Cy3-CatSseq-BHQ-2 DOTAM (4, $\left.5.4 \mathrm{mg}, 2.4 \mu \mathrm{mol}\right)$ was dissolved in acetonitrile $(2 \mathrm{ml})$ and triethylamine $(2.1 \mu \mathrm{l}$, $14 \mu \mathrm{mol})$ and acetic anhydride $(0.9 \mu \mathrm{l}, 9.6 \mu \mathrm{mol})$ were added and the reaction was stirred at r.t. for $18 \mathrm{~h}$. Water $(2 \mathrm{ml})$ was added and the crude solution was purified by HPLC (5-75\% ACN in $22 \mathrm{~min}, 75-100 \%$ in $4 \mathrm{~min})$ to yield $3 \mathrm{mg}(55 \%)$ of the title compound 6 as purple powder after lyophilization. HRMS (pos. ESI-TOF): $m / z$ calculated for $\mathrm{C}_{125} \mathrm{H}_{192} \mathrm{~N}_{25} \mathrm{O}_{27}[\mathrm{M}+\mathrm{H}]^{2+}$ 1238.7258 , found 1238.7250 .

\section{Bis-(PEGNHsucc)-Cy3-CatSseq-BHQ-2 DOTAM (S22)}

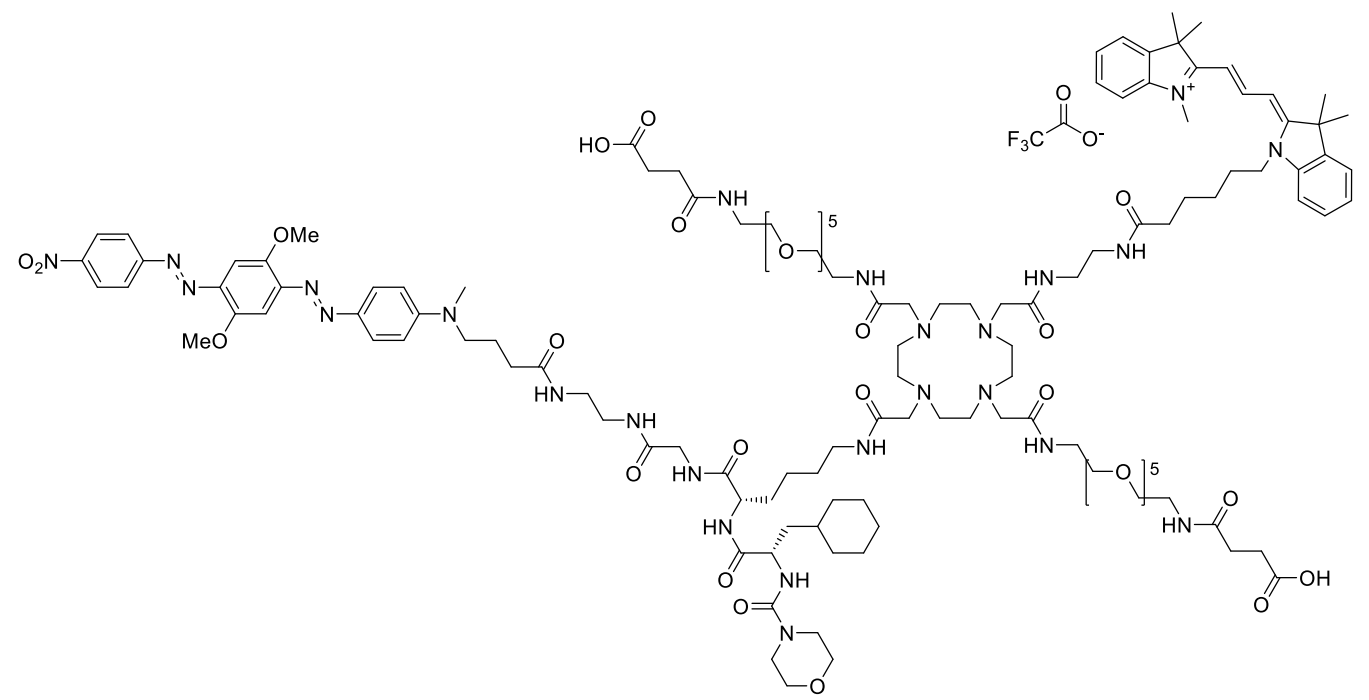

Bis-(PEGNH $)_{2}$-Cy3-CatSseq-BHQ-2 DOTAM $(4,14 \mathrm{mg}, 5.3 \mu \mathrm{mol})$ was dissolved in acetonitrile $(3 \mathrm{ml})$ and triethylamine $(5.5 \mu \mathrm{l}$, $40.0 \mu \mathrm{mol})$ and succinic anhydride $(2.0 \mathrm{mg}, 20.0 \mu \mathrm{mol})$ were added and the reaction was stirred at r.t. for $18 \mathrm{~h}$. Water $(1 \mathrm{ml})$ was added and the crude solution was purified by HPLC (5-75\% ACN in $22 \mathrm{~min}, 75-100 \%$ in $4 \mathrm{~min})$ to yield $13 \mathrm{mg}(86 \%)$ of the title compound $\mathbf{S} 22$ as purple powder after lyophilization. 
Bis-(cRGDfK)-Cy3/BHQ-2 CatS activatable probe (5)

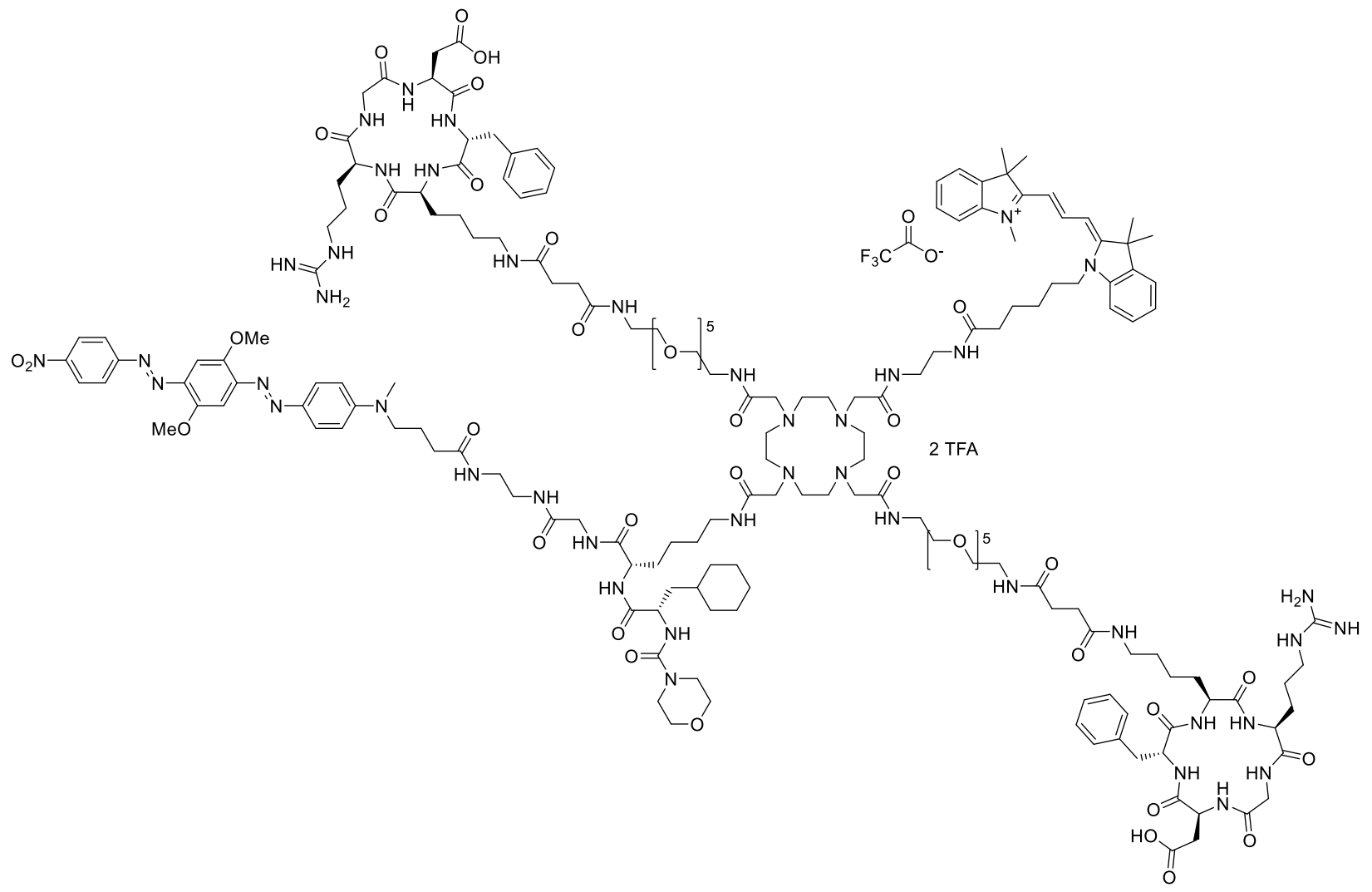

Bis-(PEGNHsucc)-Cy3-CatSseq-BHQ-2 DOTAM (S22, $7 \mathrm{mg}, 2.7 \mu \mathrm{mol}$ ) was dissolved in DMF (4 ml) and DIPEA (3.7 $\mu$, $21.6 \mu \mathrm{mol})$ and HATU $(2.1 \mathrm{mg}, 5.4 \mu \mathrm{mol})$ were added. The solution was stirred for $10 \mathrm{~min}$, then cR(Pbf)GD(tBu)fK peptide (S4, $6.7 \mathrm{mg}, 7.3 \mu \mathrm{mol}$ ) was added and the reaction was stirred for $18 \mathrm{~h}$ at r.t. The solvents were evaporated under reduced pressure and TFA/DCM $/ \mathrm{H}_{2} \mathrm{O}(50: 50: 1,5 \mathrm{ml})$ was added and the solution was stirred for $4 \mathrm{~h}$ at r.t. The solvents were evaporated under reduced pressure and the crude was purified by HPLC $(5-100 \%$ ACN in $26 \mathrm{~min})$ to yield $8 \mathrm{mg}(76 \%)$ of the title compound 5 as purple powder after lyophilization. HRMS (pos. ESI-TOF): $m / z$ calculated for $\mathrm{C}_{183} \mathrm{H}_{274} \mathrm{~N}_{43} \mathrm{O}_{43}[\mathrm{M}+3 \mathrm{H}]^{4+} 941.7725$, found 941.7712 


\section{Probe Characterization}

\section{Activation of probes 5 and 6 by cathepsin $S$ in vitro}

Probe 5

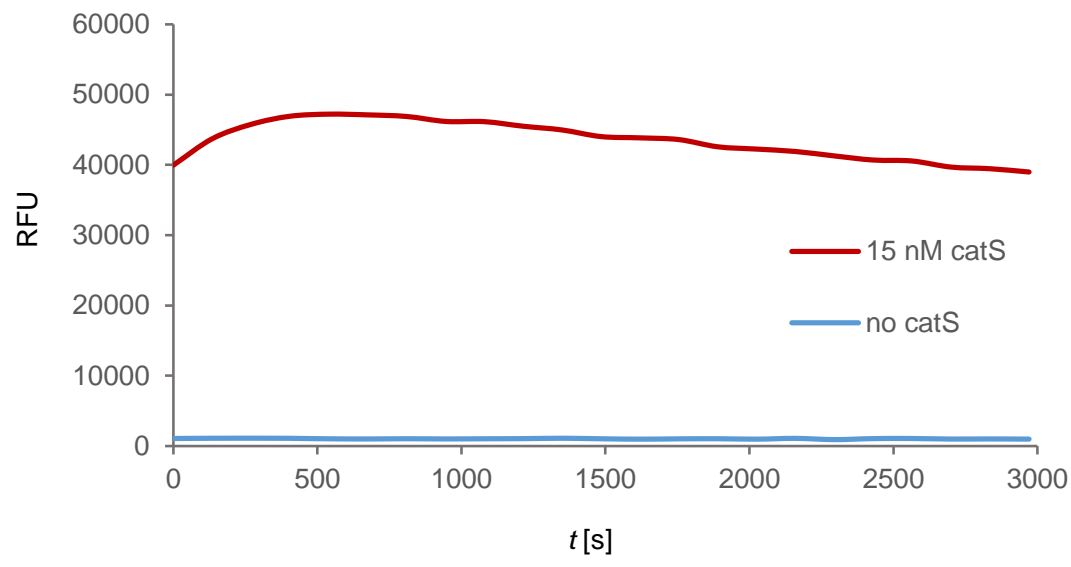

Probe 6

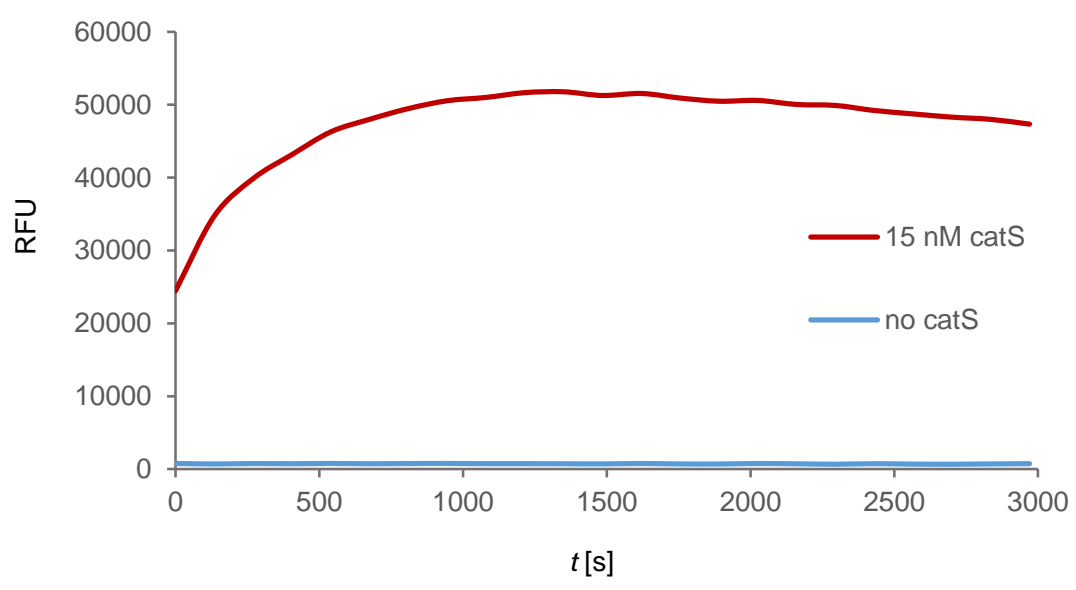

Figure S1: Activation of quenched probes 5 and $\mathbf{6}(1.5 \mu \mathrm{M})$ by cathepsin $\mathrm{S}(15 \mathrm{nM})$ in buffer (150 mM sodium acetate/HEPES pH 6.5 , $300 \mathrm{mM} \mathrm{NaCl}, 0.001 \%$ Tween20 and $1 \mathrm{mM} \mathrm{DTT} ; \lambda_{\mathrm{ex}} / \lambda_{\mathrm{em}}=550 / 570 \mathrm{~nm}, 7 \mathrm{~nm}$ bandwidth). Experiments conducted in duplicate. 


\section{LCMS cleavage traces of probes 5 and 6 with cathepsin S}

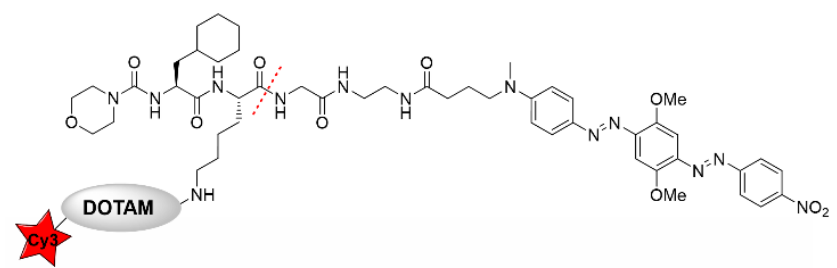

targeted activatable probe 5

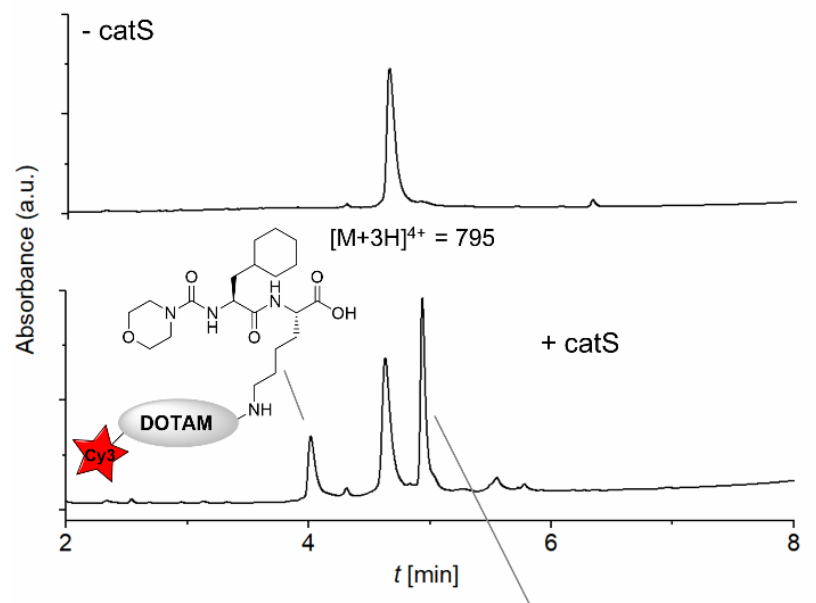

non-targeted control 6

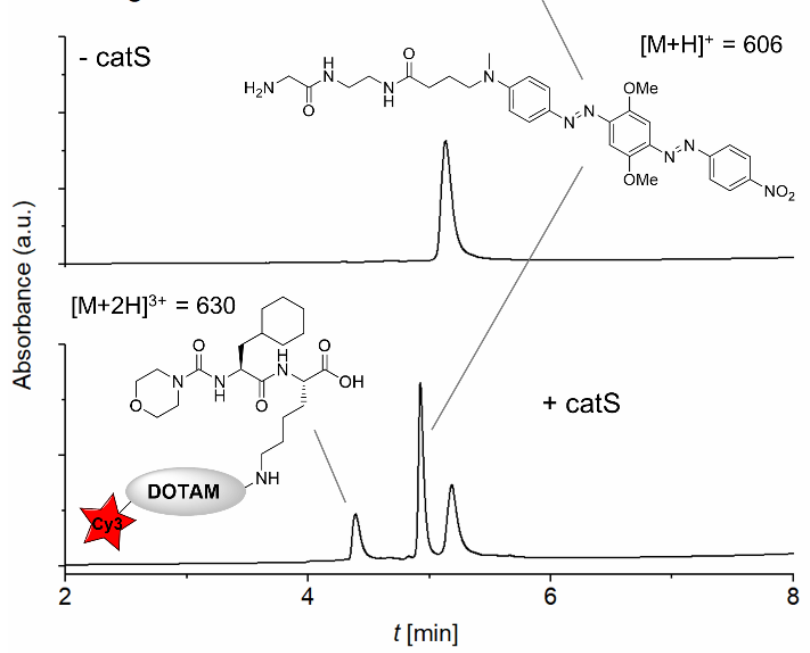

Figure S2:LCMS traces of probe 5 and control 6 (schematic representation above with marked cleavage site) upon incubation with cathepsin S (ca. $10 \mathrm{nM}$ ) in buffer (150 mM sodium acetate/HEPES pH 6.5, $300 \mathrm{mM} \mathrm{NaCl}, 0.001 \%$ Tween20 and $1 \mathrm{mM}$ DTT) and corresponding detected fragment masses. 


\section{Cleavage Kinetics}

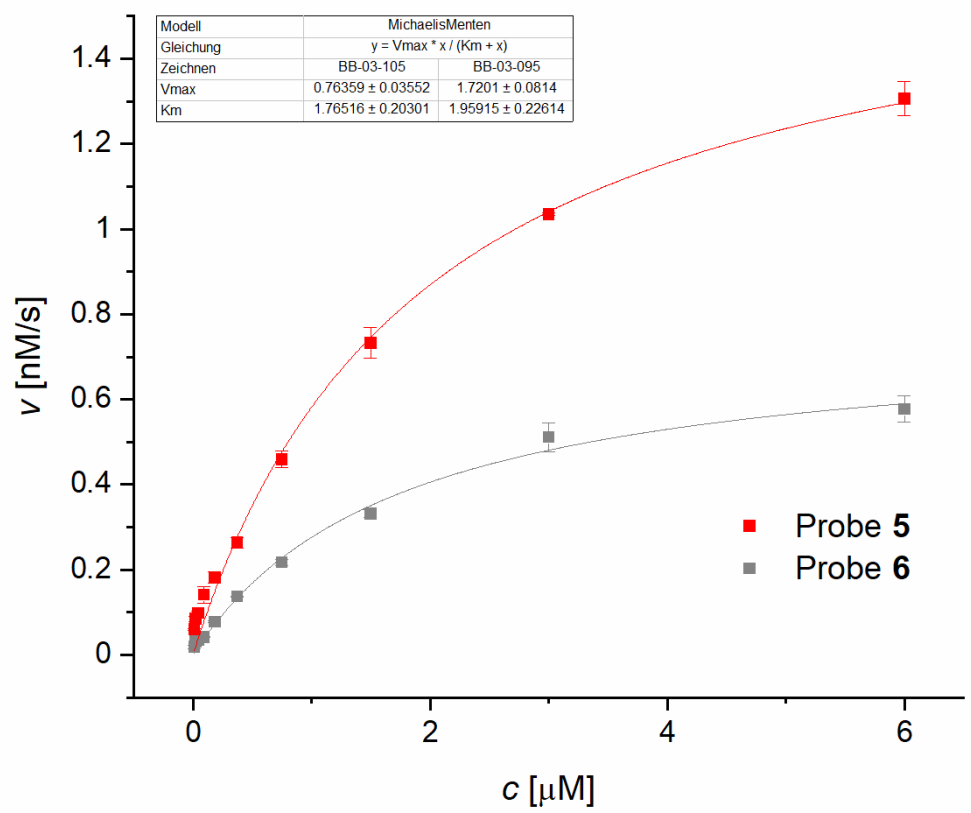

Figure S3:Concentration versus initial velocity for cleavage of probe 5 and control probe 6 by cathepsin S $(0.8 \mathrm{nM})$ in buffer (150 mM sodium acetate/HEPES pH 6.5, $300 \mathrm{mM} \mathrm{NaCl}, 0.001 \%$ Tween20 and $1 \mathrm{mM} \mathrm{DTT} ; \lambda_{\mathrm{ex}} / \lambda_{\mathrm{em}}=550 / 570 \mathrm{~nm}, 7 \mathrm{~nm}$ bandwidth). Data are fitted to Michaelis-Menten-equation to obtain parameters $K_{\mathrm{M}}$ and $v_{\max }$. Experiments conducted in duplicate. 


\section{In vitro inhibition of cathepsin S}

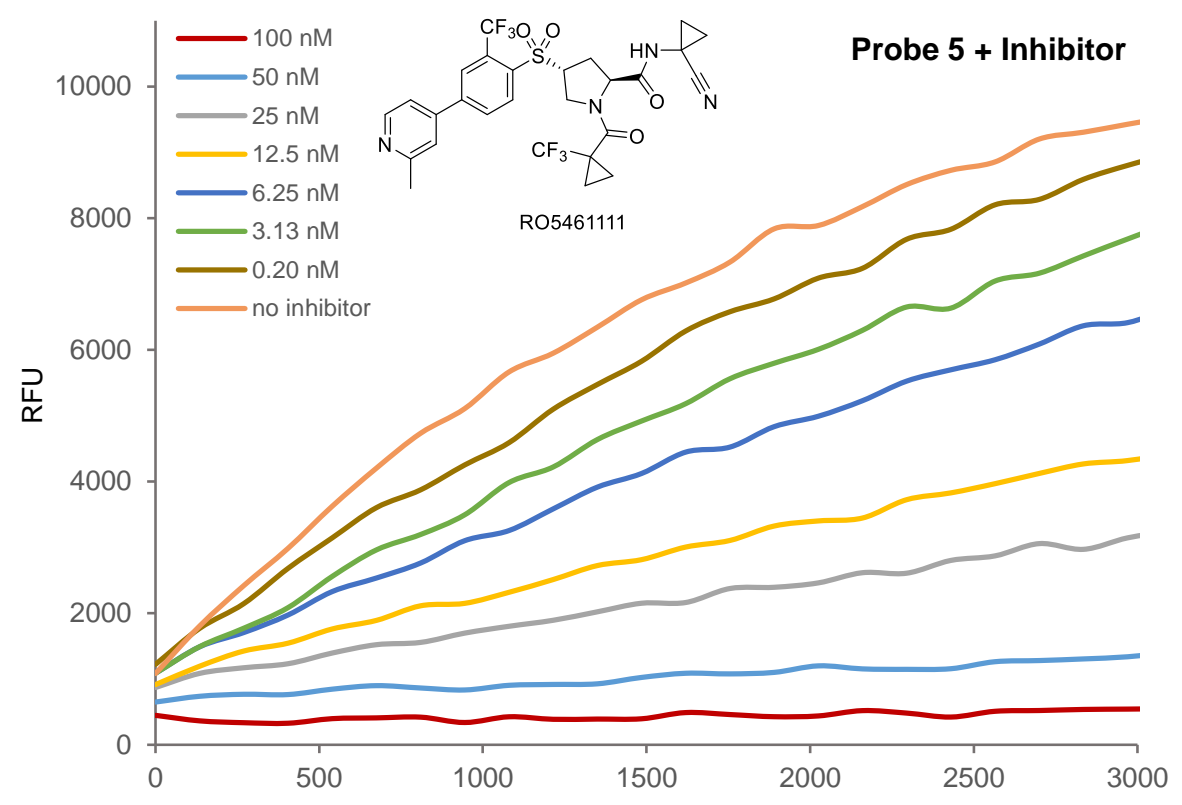

$t[\mathrm{~s}]$

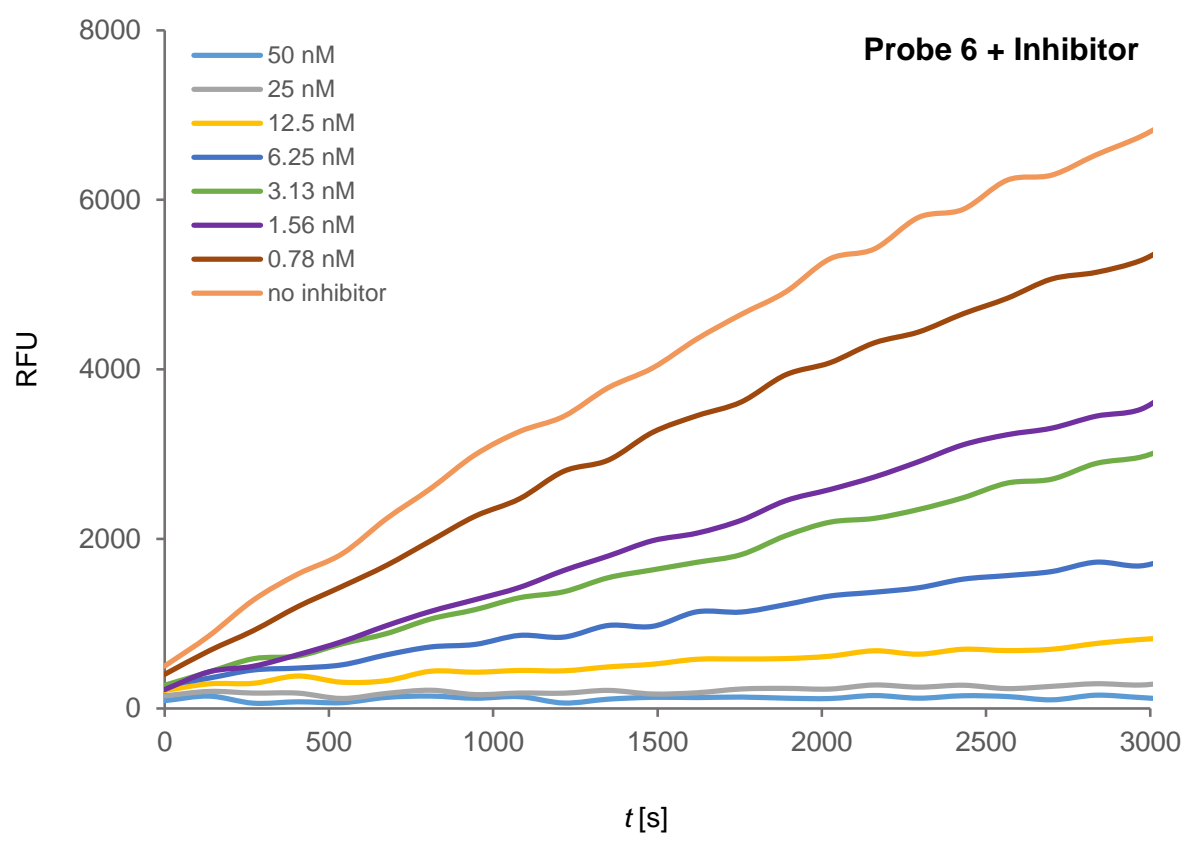

Figure S4: Inhibition of probe activation employing the selective cathepsin S inhibitor RO5461111 (1 $\mu \mathrm{M}$ probe, $1.5 \mathrm{nM}$ cathepsin $\mathrm{S}$ in buffer: $150 \mathrm{mM}$ sodium acetate/HEPES pH 6.5, $300 \mathrm{mM} \mathrm{NaCl}, 0.001 \%$ Tween20 and $1 \mathrm{mM} \mathrm{DTT} ; \lambda_{\mathrm{ex}} / \lambda_{\mathrm{em}}=550 / 570 \mathrm{~nm}, 7 \mathrm{~nm}$ bandwidth). Experiments conducted in duplicate. 
Probe response to various intracellular species
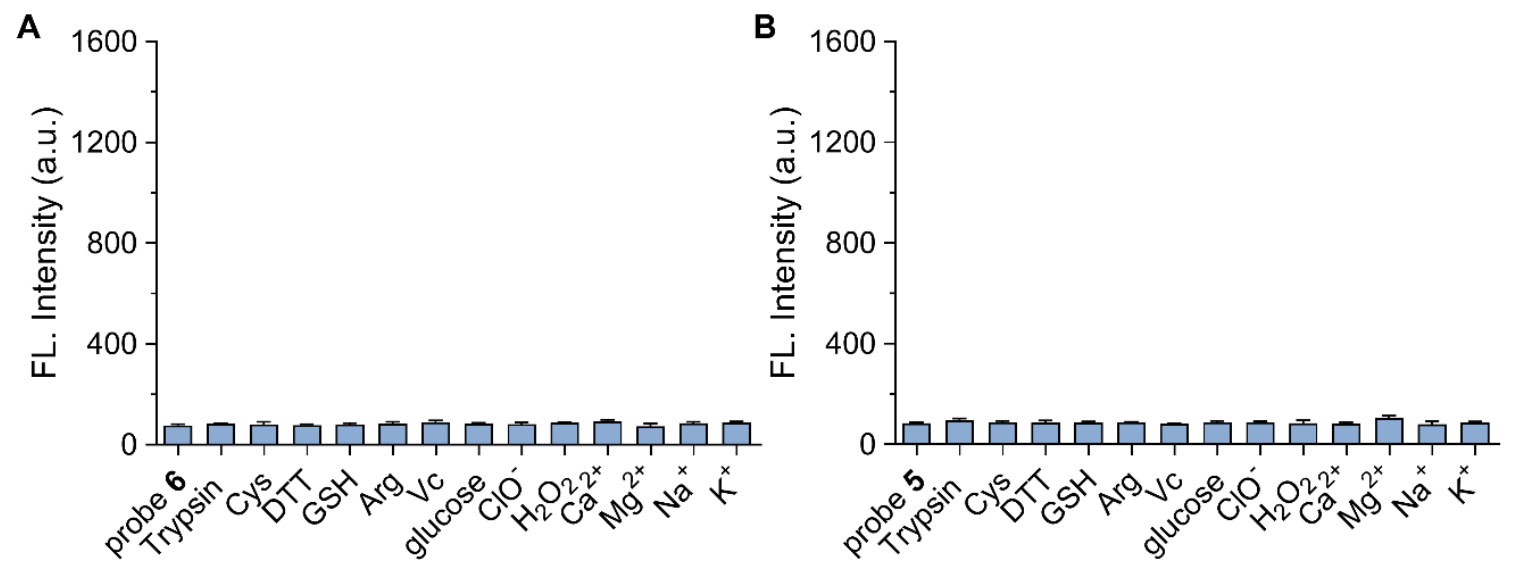

Figure S5: Responses of $1 \mu \mathrm{M}$ probe 5 (A) or probe $\mathbf{6}$ (B) to various intracellularly occurring species after $1 \mathrm{~h}$ incubation in $150 \mathrm{mM}$ HEPES pH 6.5: trypsin $(10 \mu \mathrm{g} / \mathrm{mL})$, cysteine $(1 \mathrm{mM})$, dithiothreitol $(1 \mathrm{mM})$, glutathione $(1 \mathrm{mM})$, arginine $(1 \mathrm{mM})$, ascorbic acid (1 mM), glucose $(10 \mathrm{mM}), \mathrm{OCl}^{-}(1 \mathrm{mM}), \mathrm{H}_{2} \mathrm{O}_{2}(10 \mu \mathrm{M}), \mathrm{CaSO}_{4}(1 \mathrm{mM}), \mathrm{MgCl}_{2}(1 \mathrm{mM}), \mathrm{KCl}(10 \mathrm{mM})$ and $\mathrm{NaCl}(10 \mathrm{mM}) . \lambda_{\mathrm{ex}} / \lambda_{\mathrm{em}}=545 / 575 \mathrm{~nm}$. Experiments conducted in duplicate.

\section{Probe response to different $\mathrm{pH}$}

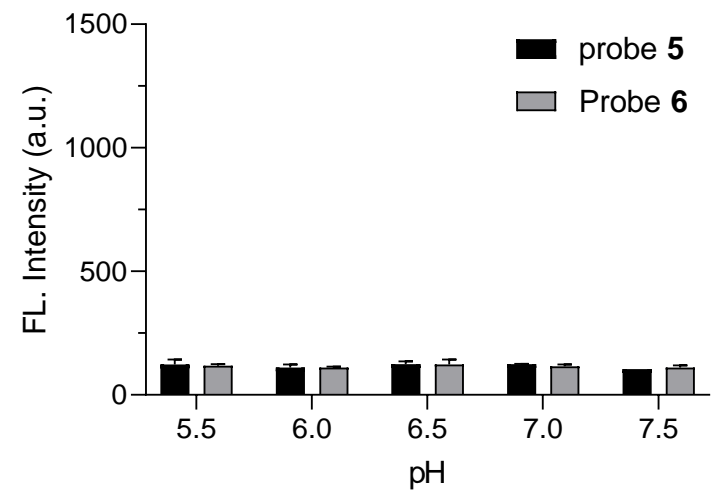

Figure S6: Response of $1 \mu \mathrm{M}$ probes 5 and 6 in $150 \mathrm{mM} \mathrm{HEPES}$ to different $\mathrm{pH}$. $\lambda_{\mathrm{ex}} / \lambda_{\mathrm{em}}=545 / 575 \mathrm{~nm}$. Experiments conducted in duplicate. 
A

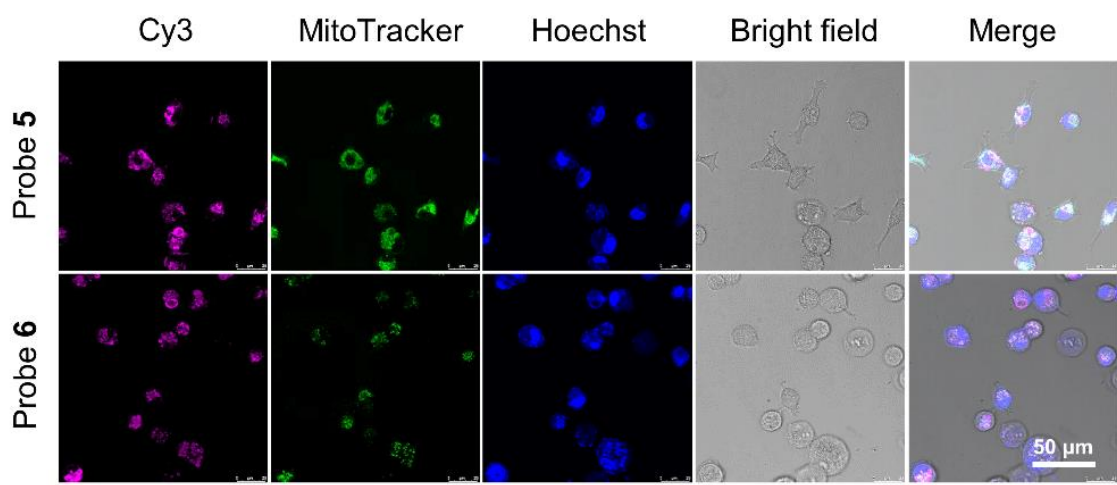

B
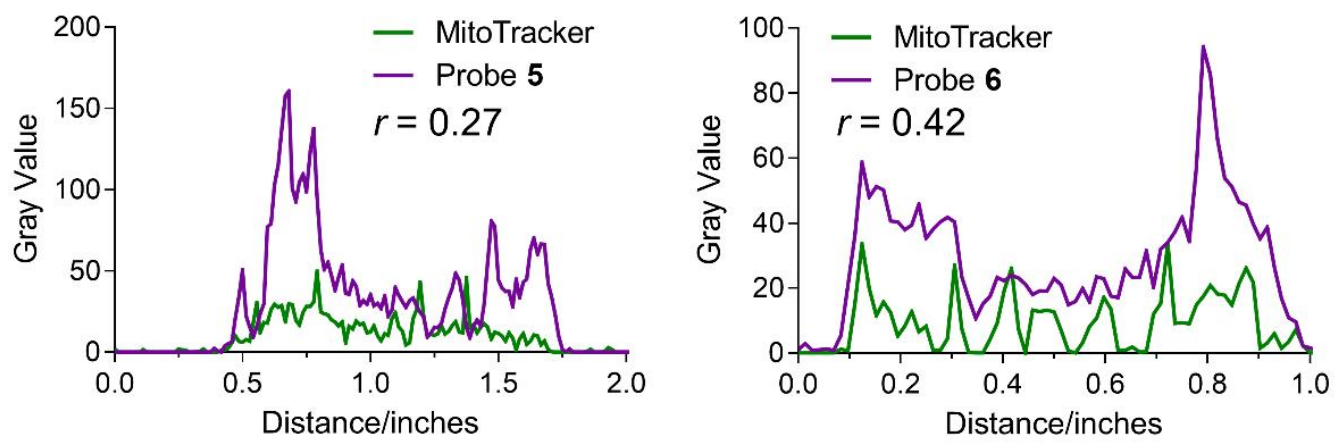

Figure S7: A. Fluorescence images of co-localization studies of probes 5 and $6(2.5 \mu \mathrm{M})$ coincubated with MitoTracker Green (200 $\mathrm{nM}$ ) in HT1080 cells for $3 \mathrm{~h}$. B. Correlation analysis for localization of probes $\mathbf{5}$ and $\mathbf{6}$ with MitoTracker and corresponding Pearson's correlation coefficient. 
Co-localization experiments of probe 5 with Lyso- and MitoTracker in MCF-7 cells

A

Су3

LysoTracker

Hoechst

Bright Field

Merge
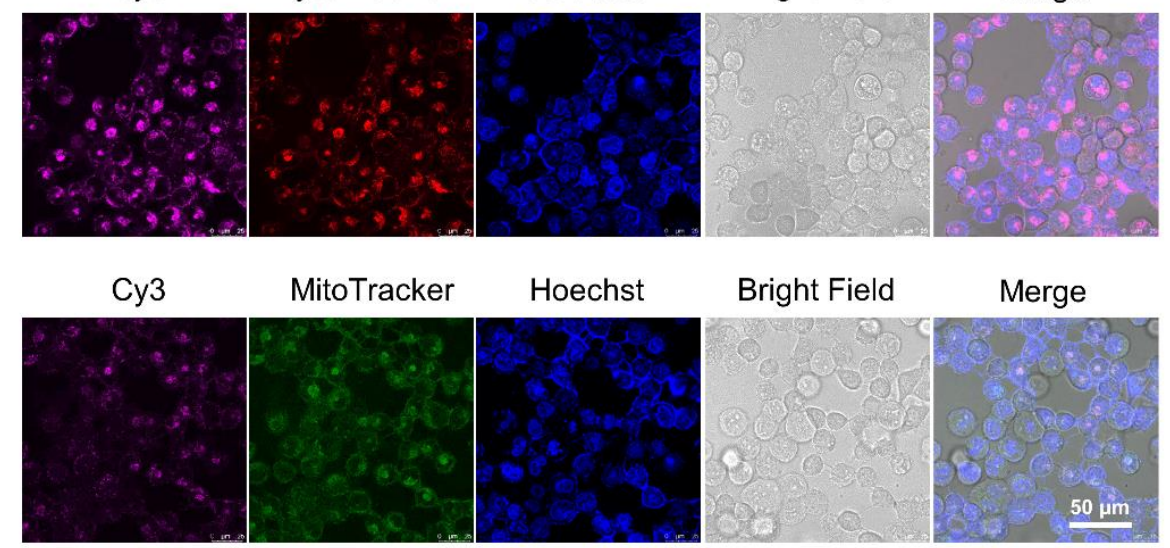

Bright Field

Merge

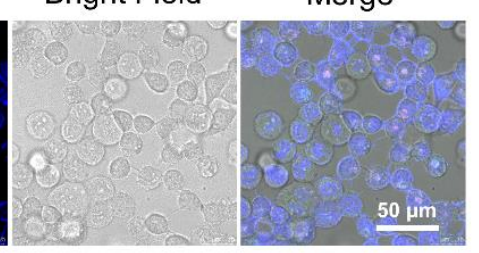

B
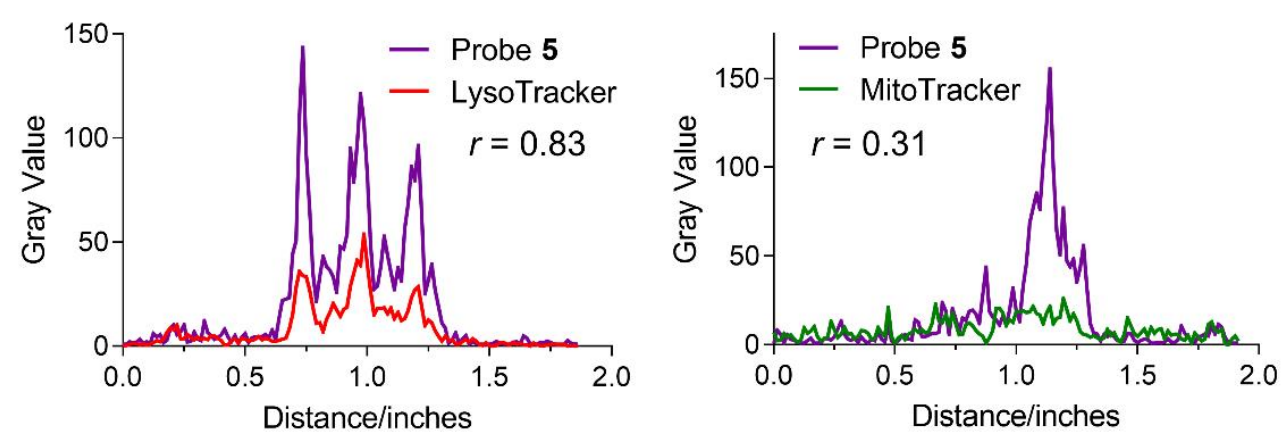

Figure S8: Fluorescence images of co-localization studies of probe $5(2.5 \mu \mathrm{M})$ coincubated with LysoTracker Red (500 nM) or MitoTracker Green (200 nM) in MCF-7 cells for 3 h. B. Correlation analysis for localization of probe 5 with Lyso- and MitoTracker and corresponding Pearson's correlation coefficient. 

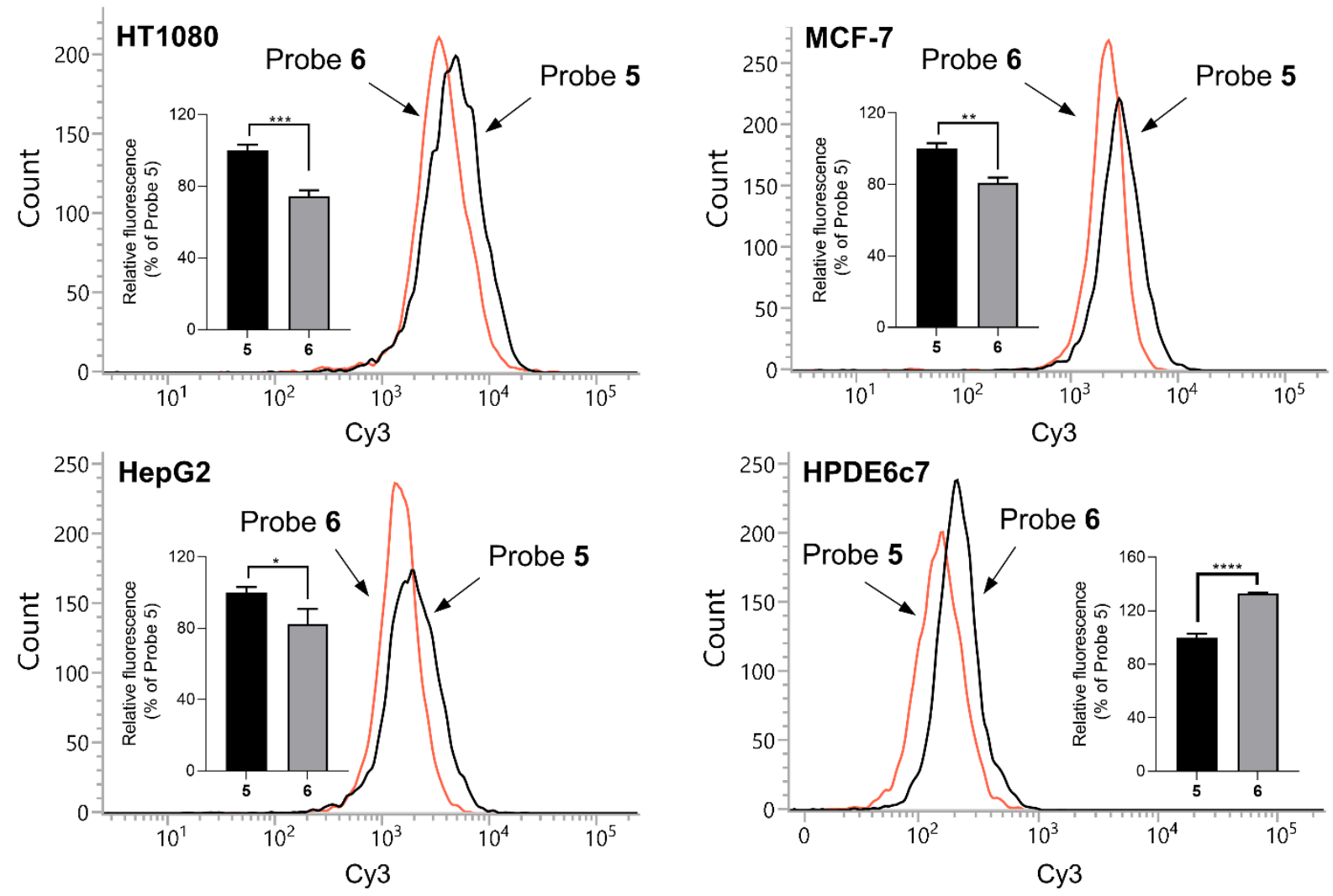

Figure S9: Flow cytometric analyses of cells labeled with probes $\mathbf{5}$ and $\mathbf{6}$. Probe $\mathbf{5}$ incubation resulted in a right shift of the cell distribution in cancer cells, indicating targeting. Quantification of mean fluorescence intensities are shown in the insets. Data shown as mean \pm s.d. $(n=3)\left({ }^{*} P<0.05,{ }^{* *} P<0.01,{ }^{* * *} P<0.001,{ }^{* * * *} P<0.0001\right)$. 


\section{References}

(1) Rauschenberg, M.; Fritz, E.-C.; Schulz, C.; Kaufmann, T.; Ravoo, B. J. Molecular recognition of surface-immobilized carbohydrates by a synthetic lectin. Beilstein Journal of Organic Chemistry 2014, 10, 1354-1364.

(2) Dai, X.; Su, Z.; Liu, J. O. An improved synthesis of a selective avß3-integrin antagonist cyclo(-RGDfK-). Tetrahedron Lett. 2000, 41, 6295-6298.

(3) Kvach, M. V.; Ustinov, A. V.; Stepanova, I. A.; Malakhov, A. D.; Skorobogatyi, M. V.; Shmanai, V. V.; Korshun, V. A. A Convenient Synthesis of Cyanine Dyes: Reagents for the Labeling of Biomolecules. Eur. J. Org. Chem. 2008, 2008, 21072117.

(4) Leriche, G.; Nothisen, M.; Baumlin, N.; Muller, C. D.; Bagnard, D.; Remy, J.-S.; Jacques, S. A.; Wagner, A. Spiro Diorthoester (SpiDo), a Human Plasma Stable Acid-Sensitive Cleavable Linker for Lysosomal Release. Bioconjugate Chem. 2015, 26, 1461-1465.

(5) Dombrowski, G. W.; Dinnocenzo, J. P.; Zielinski, P. A.; Farid, S.; Wosinska, Z. M.; Gould, I. R. Efficient Unimolecular Deprotonation of Aniline Radical Cations. J. Org. Chem. 2005, 70, 3791-3800.

(6) Passemard, S.; Staedler, D.; Učňová, L.; Schneiter, G. S.; Kong, P.; Bonacina, L.; Juillerat-Jeanneret, L.; Gerber-Lemaire, S. Convenient synthesis of heterobifunctional poly(ethylene glycol) suitable for the functionalization of iron oxide nanoparticles for biomedical applications. Bioorg. Med. Chem. Lett. 2013, 23, 5006-5010.

(7) De León-Rodríguez, L. M.; Kovacs, Z.; Esqueda-Oliva, A. C.; Miranda-Olvera, A. D. Highly regioselective N-trans symmetrical diprotection of cyclen. Tetrahedron Lett. 2006, 47, 6937-6940.

\section{Appendix: NMR spectra and LCMS traces of new compounds and key compounds}




\section{Cathepsin S Sequence (S1)}

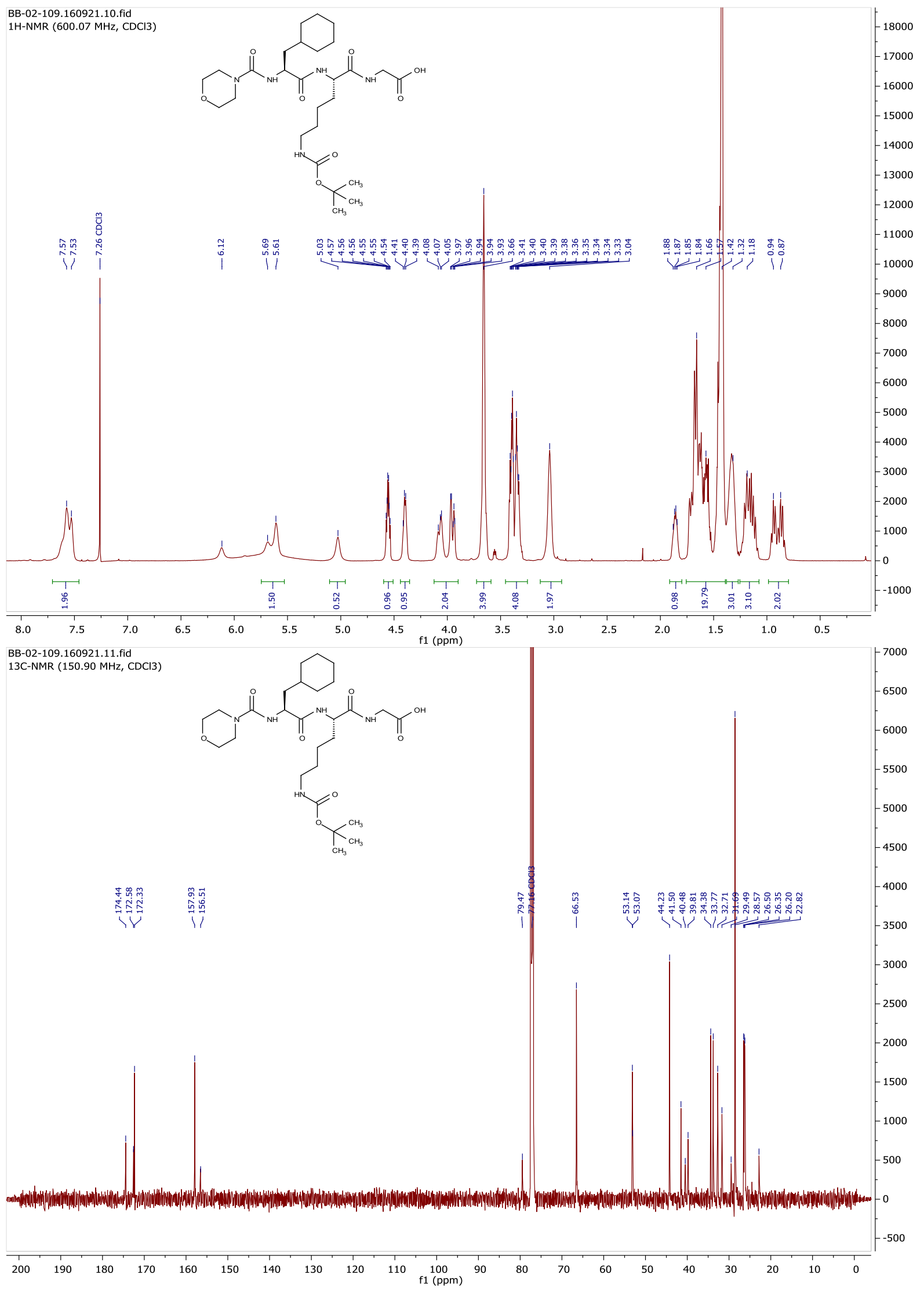




\section{H-D(tBu)-f-K(Cbz)-R(Pbf)-G-OH (S2)}

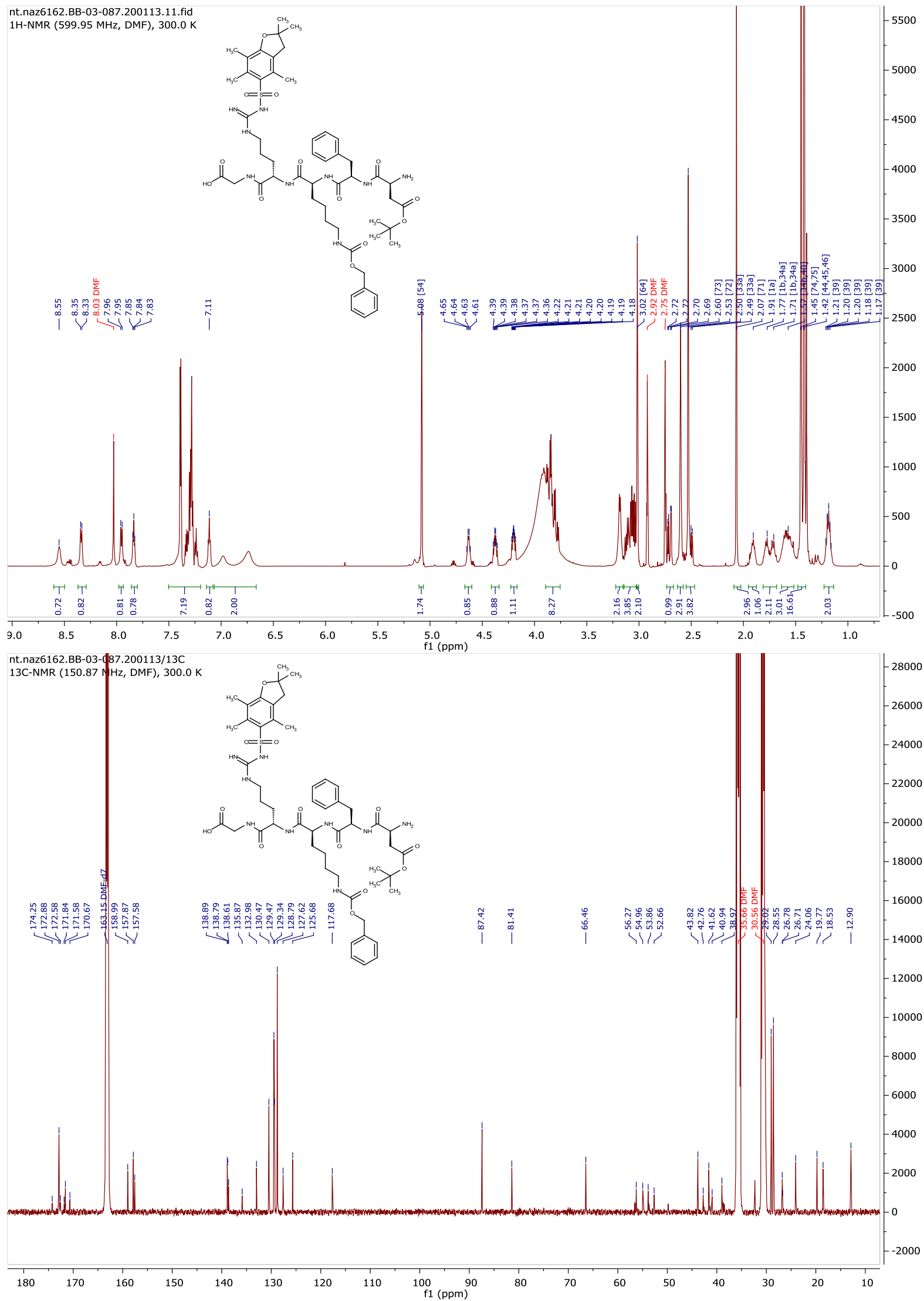




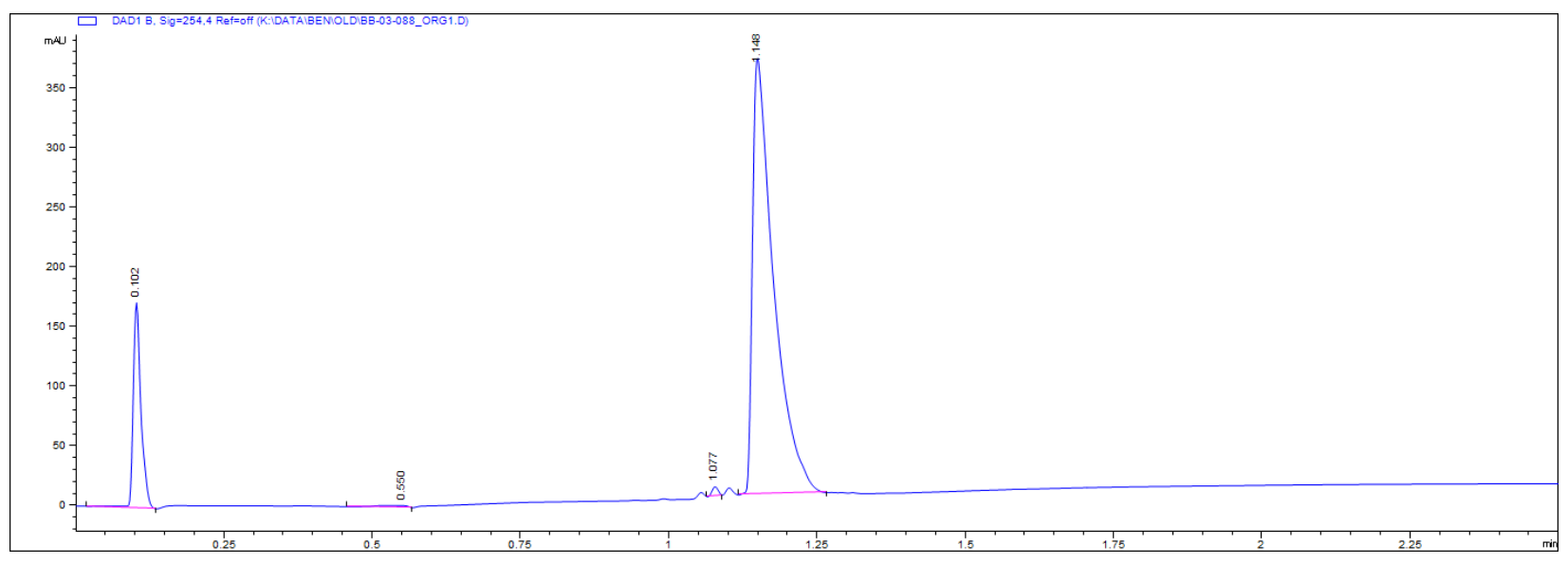


$\mathrm{cR}(\mathrm{Pbf}) \mathrm{GD}(\mathrm{tBu}) \mathrm{fK}(\mathrm{Cbz})(\mathrm{S} 3)$

BB-04-134F1.200123.10.fid 1H-NMR (599.95 MHz, DMF)
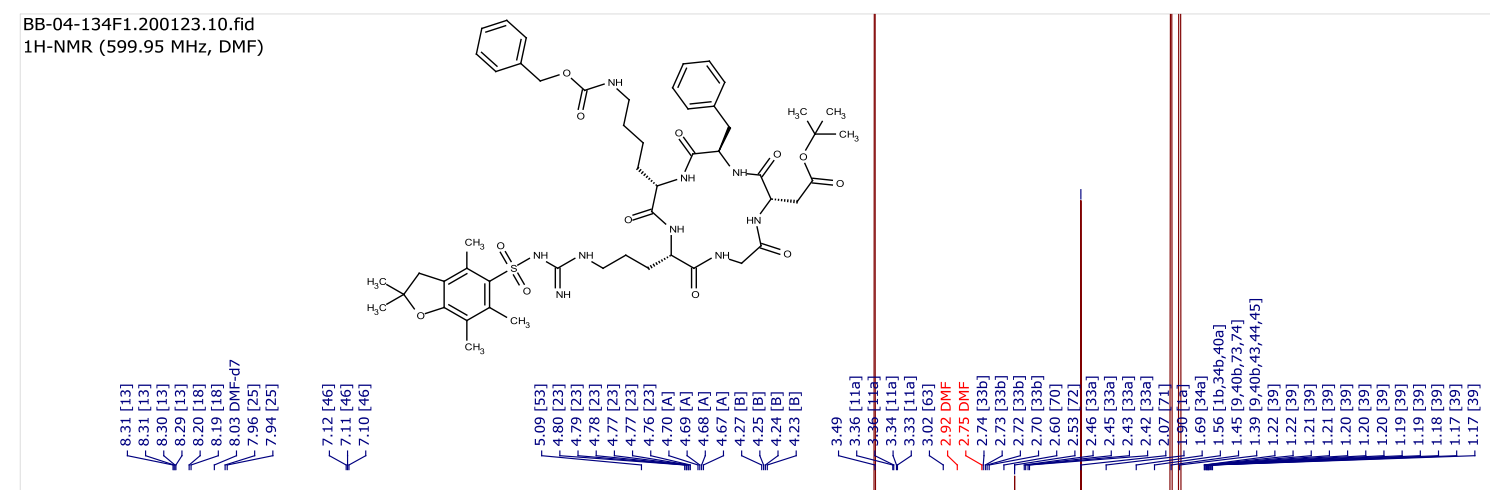

22000 21000 20000 9000 8000 7000 6000 5000 4000 3000 2000 11000 10000 9000 $-8000$ 7000 6000 $-5000$ $-4000$ $-3000$ $-2000$ $-1000$

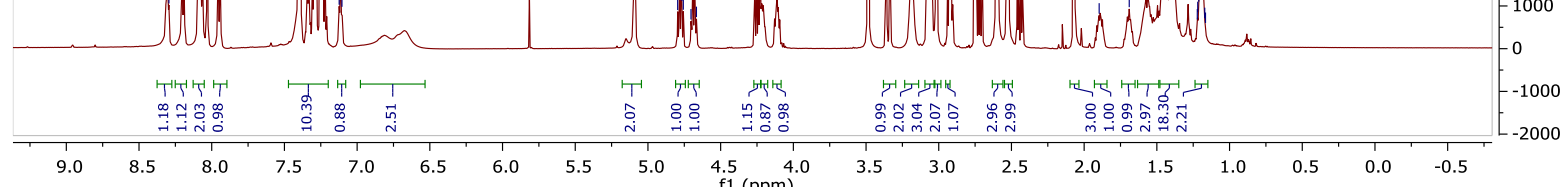

BB-04-134F1.200123.11 fid 13C-NMR (150.87 MHz, DMF)

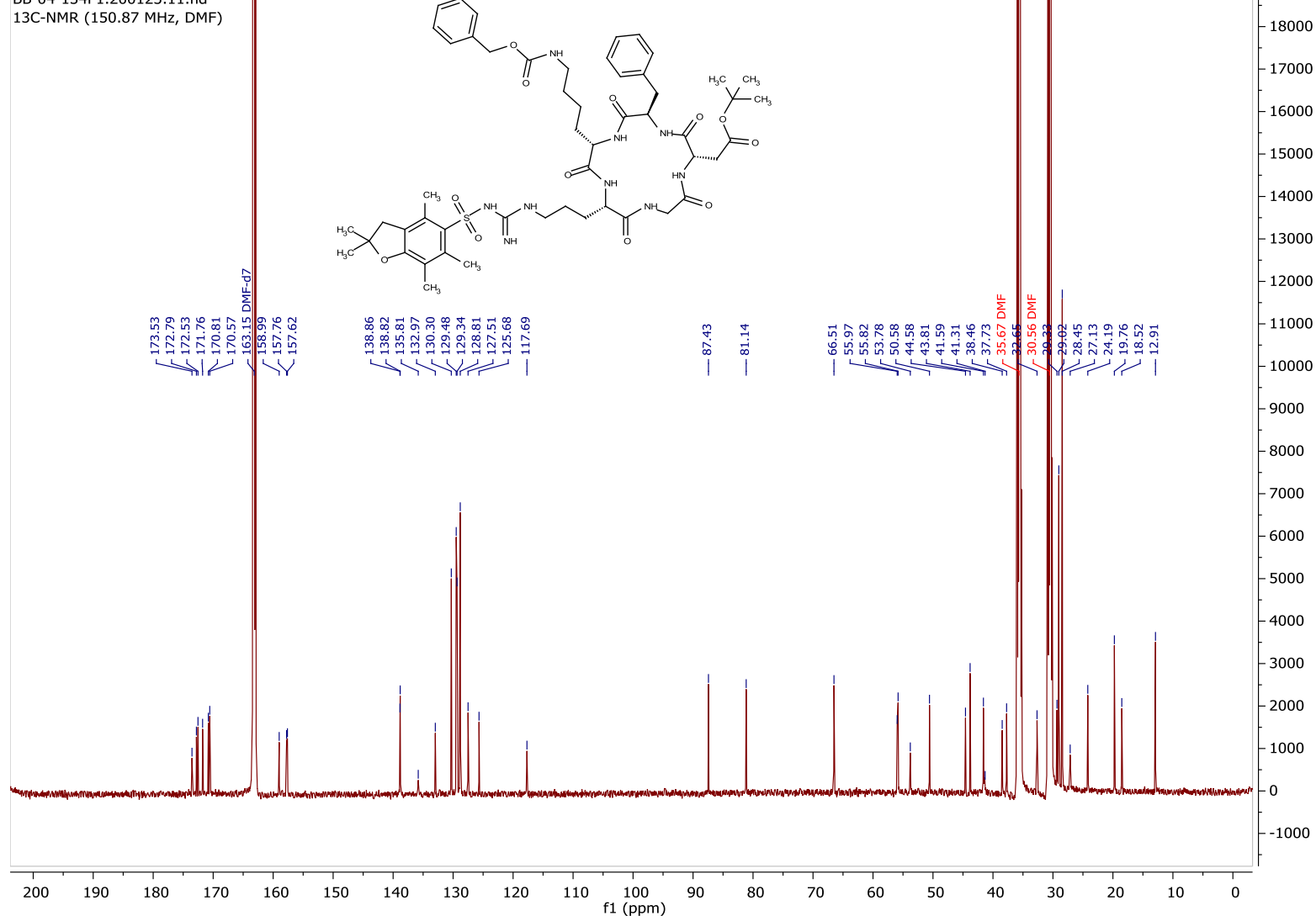




\section{$\mathrm{cR}(\mathrm{Pbf}) \mathrm{GD}(t \mathrm{Bu}) \mathrm{fK}(\mathrm{S} 4)$}

BB-04-137.200214.10.fid 1H-NMR (599.95 MHz, DMF)

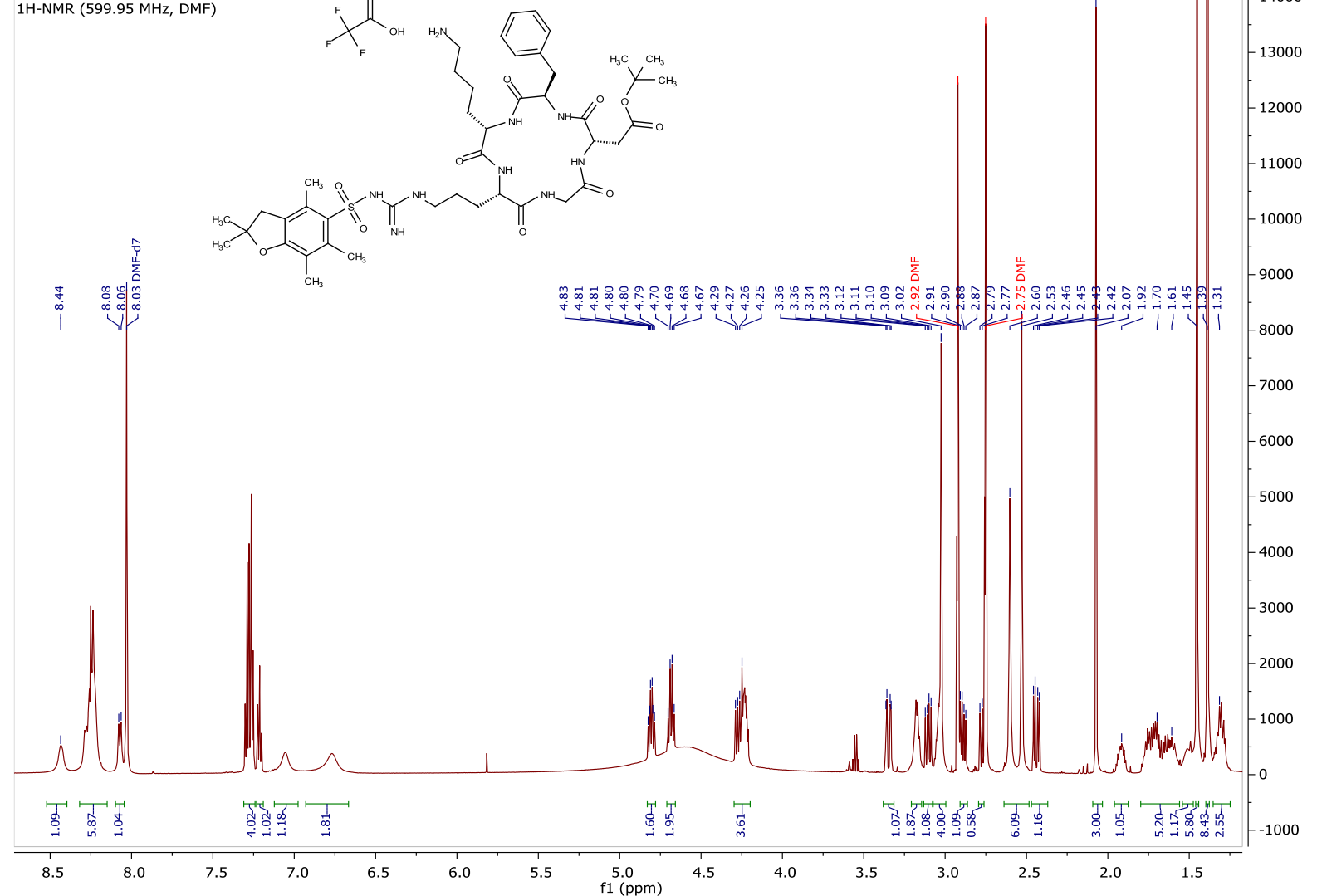

BB-04-137.20021/4.11.fid 13C-NMR (150.8) MHz, DMF)
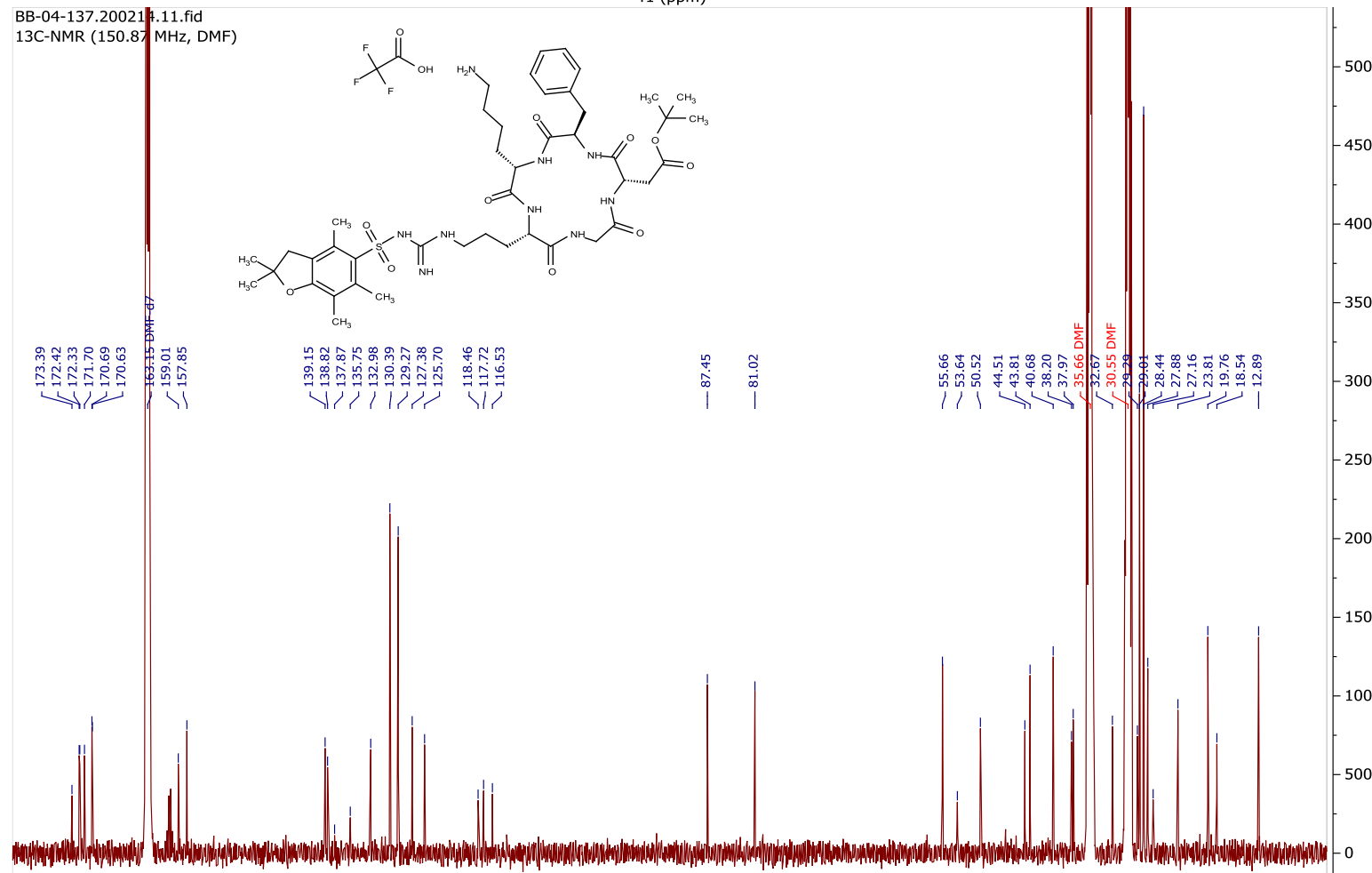

5000

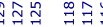

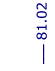

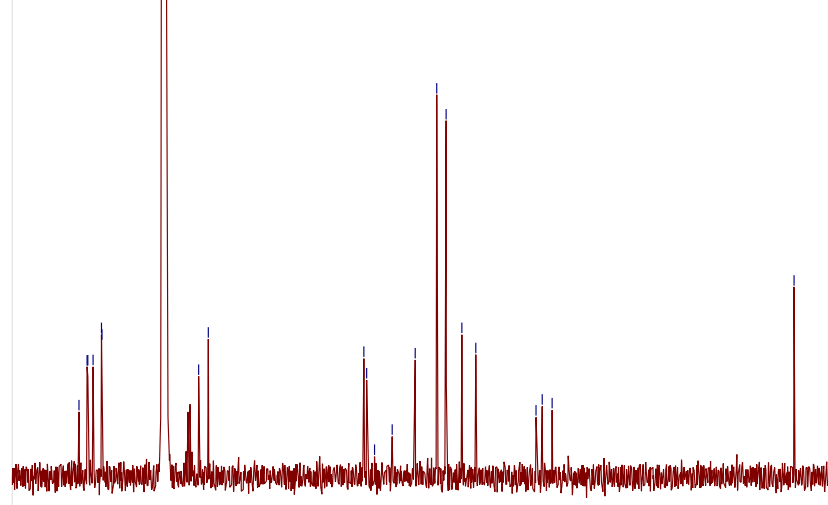

(1)

\section{-}

$180 \quad 170$

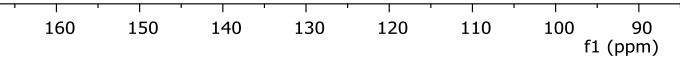




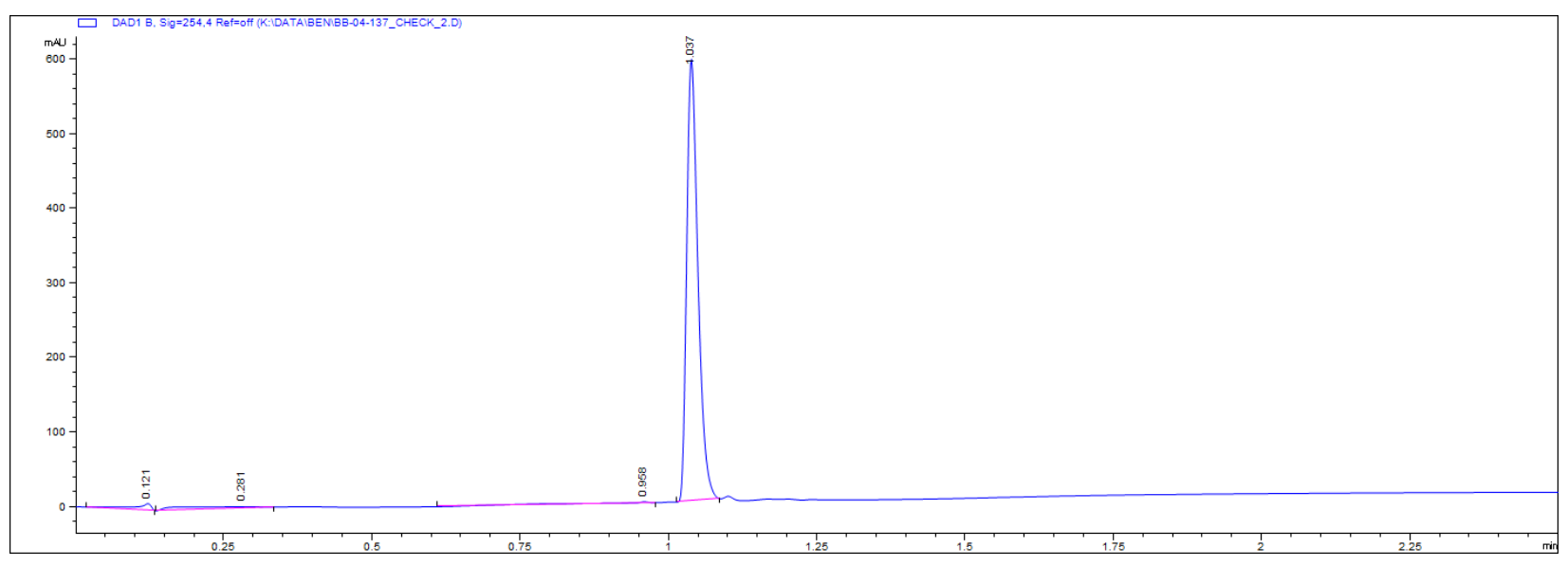




\section{Cy3 (S7)}

BB-02-121.2.160905.10.fid

1H-NMR (300.18 MHz, CDCl3)

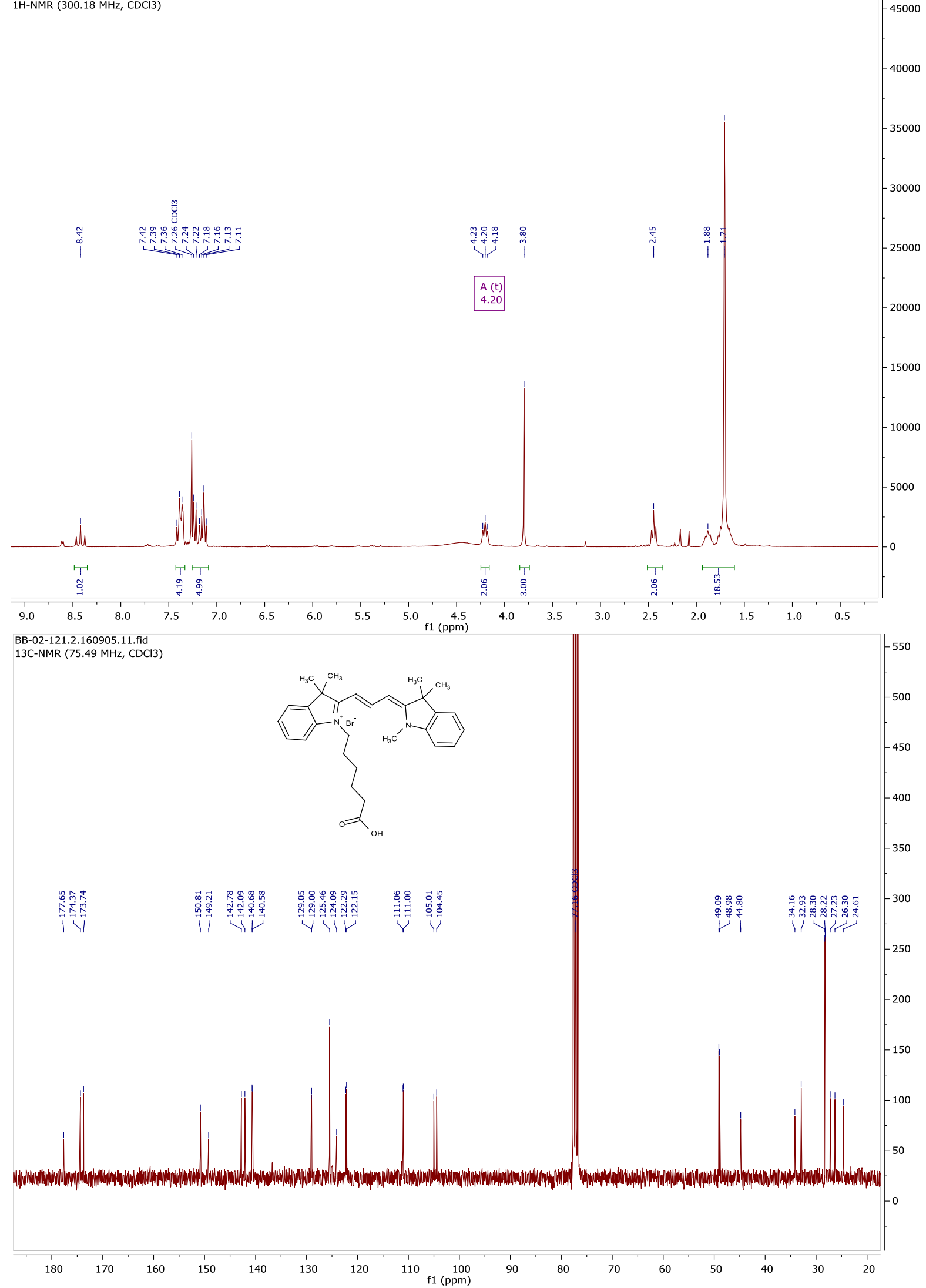


Cy3-EDABoc (S8a)

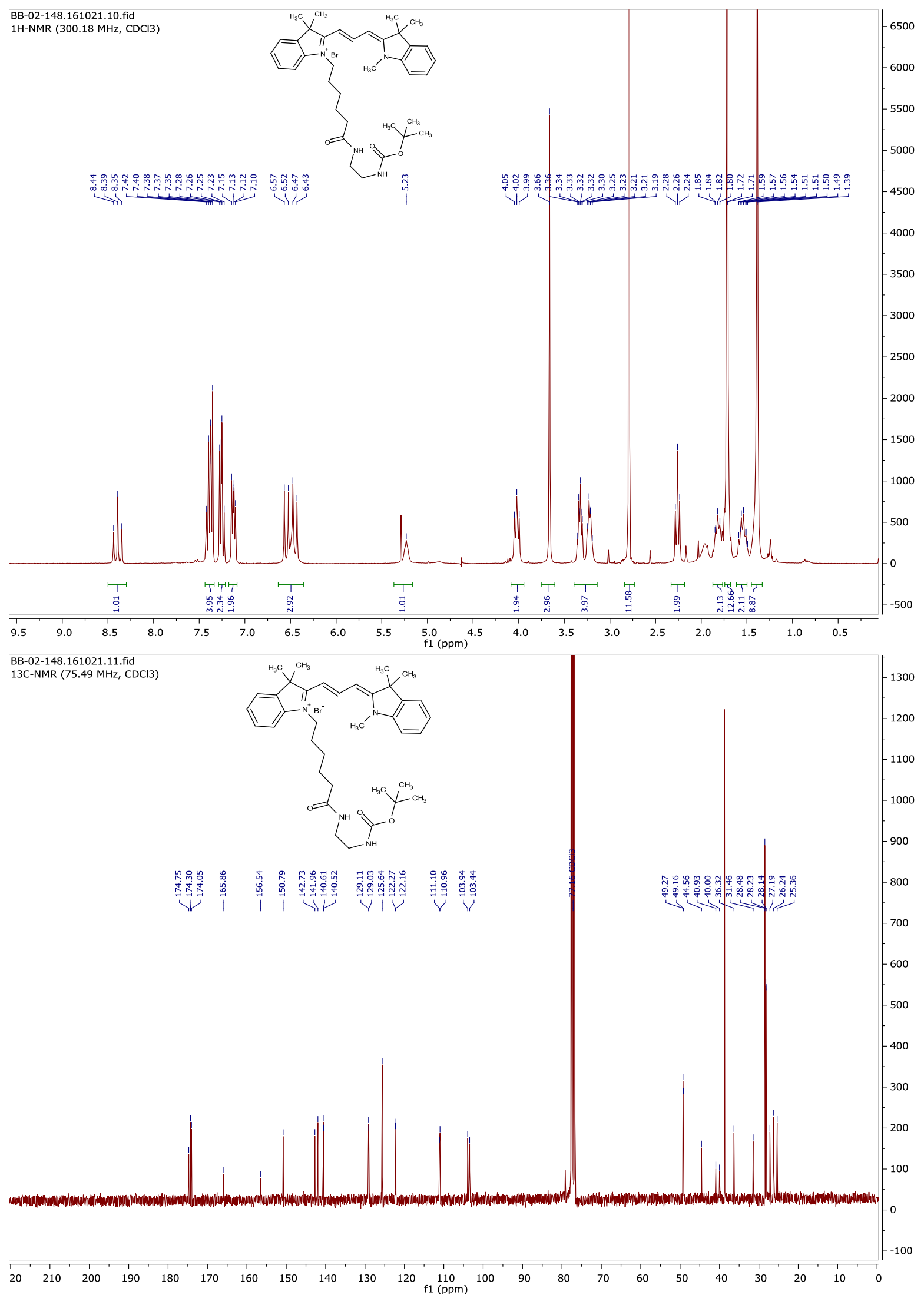


BHQ-2 (S10)

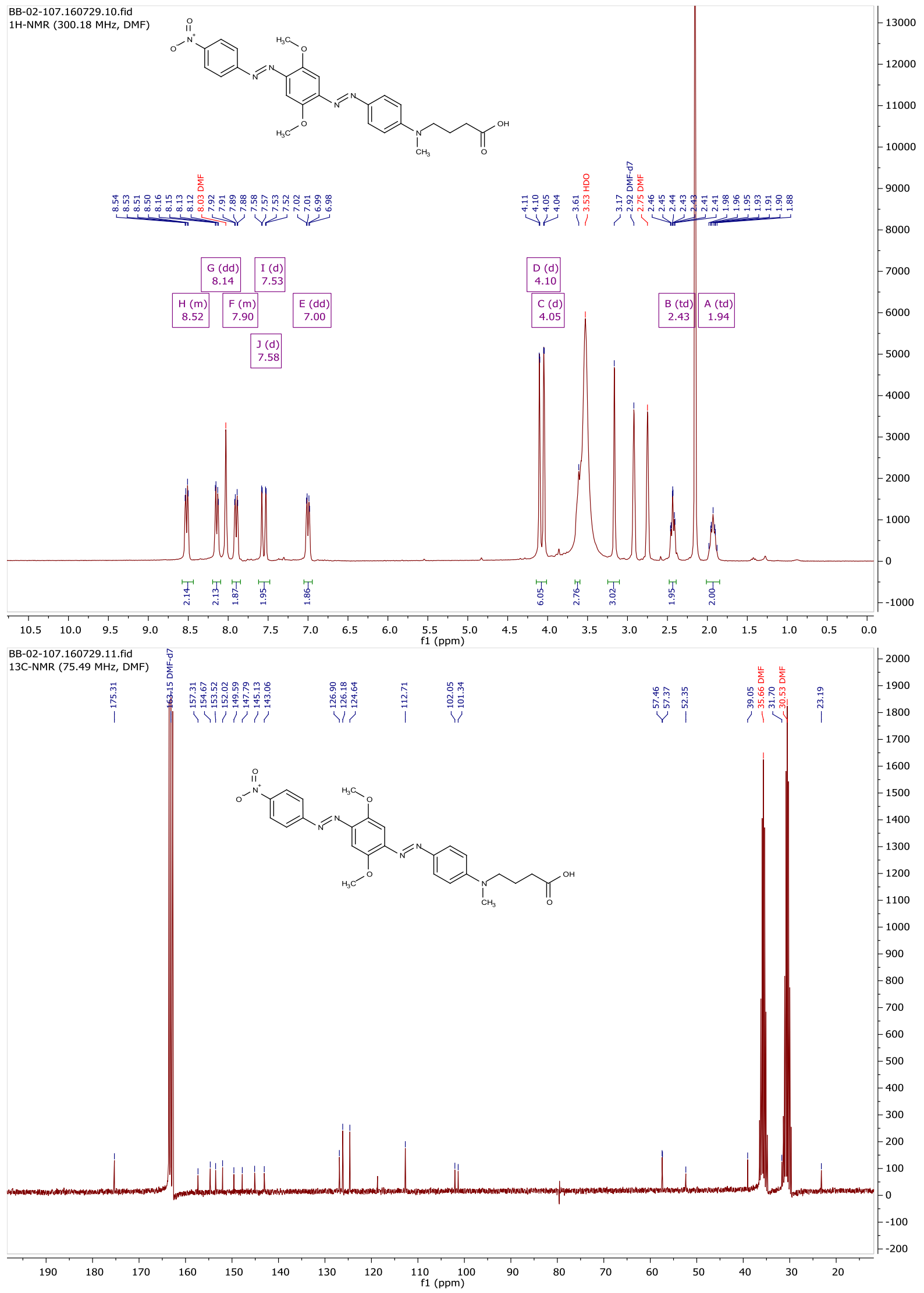


BHQ-2-EDABoc (S11a)

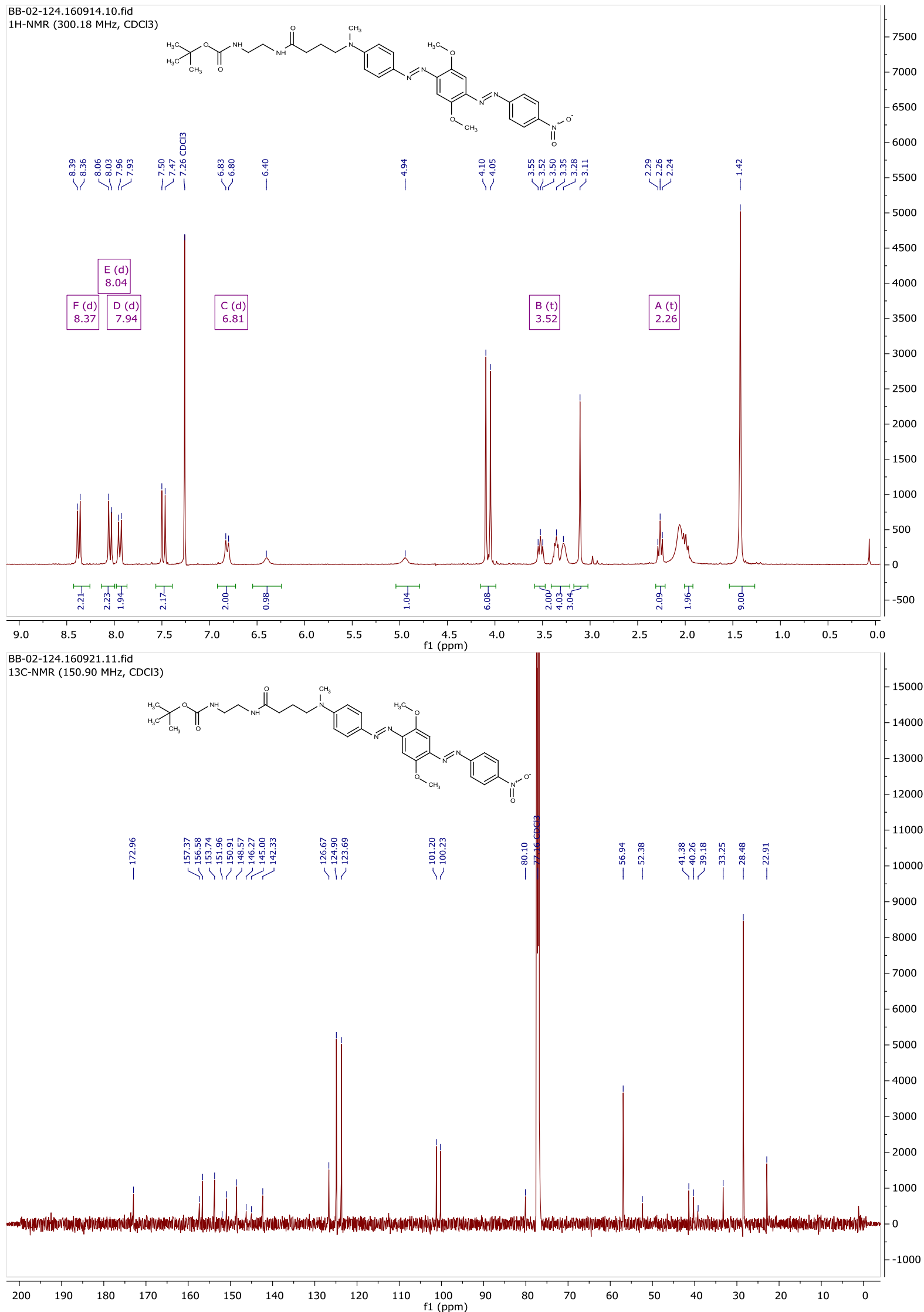


Boc-protected CatS Sequence-BHQ-2 (S12a)

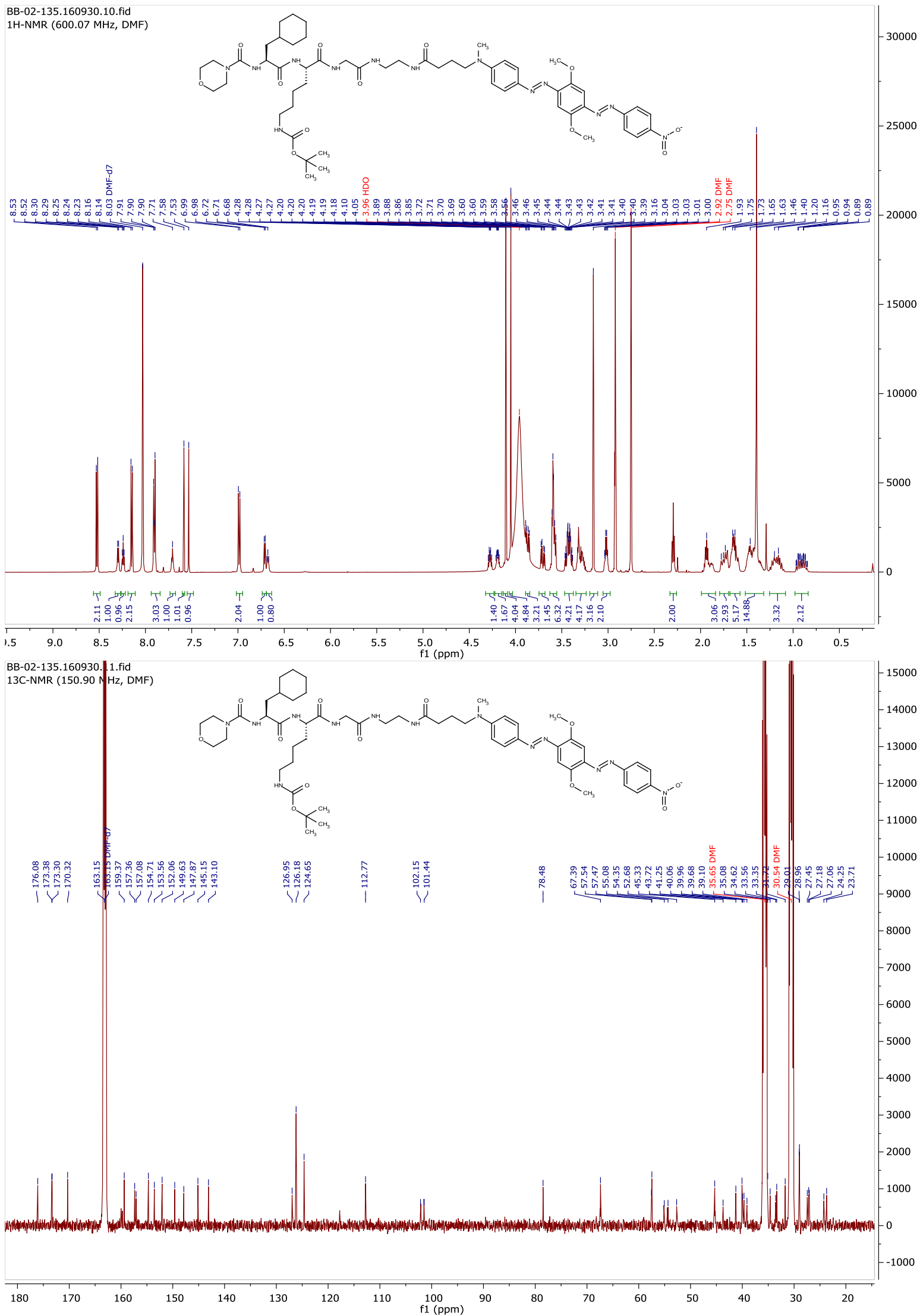


tert-Butyl (17-amino-3,6,9,12,15-pentaoxaheptadecyl)carbamate (S17)

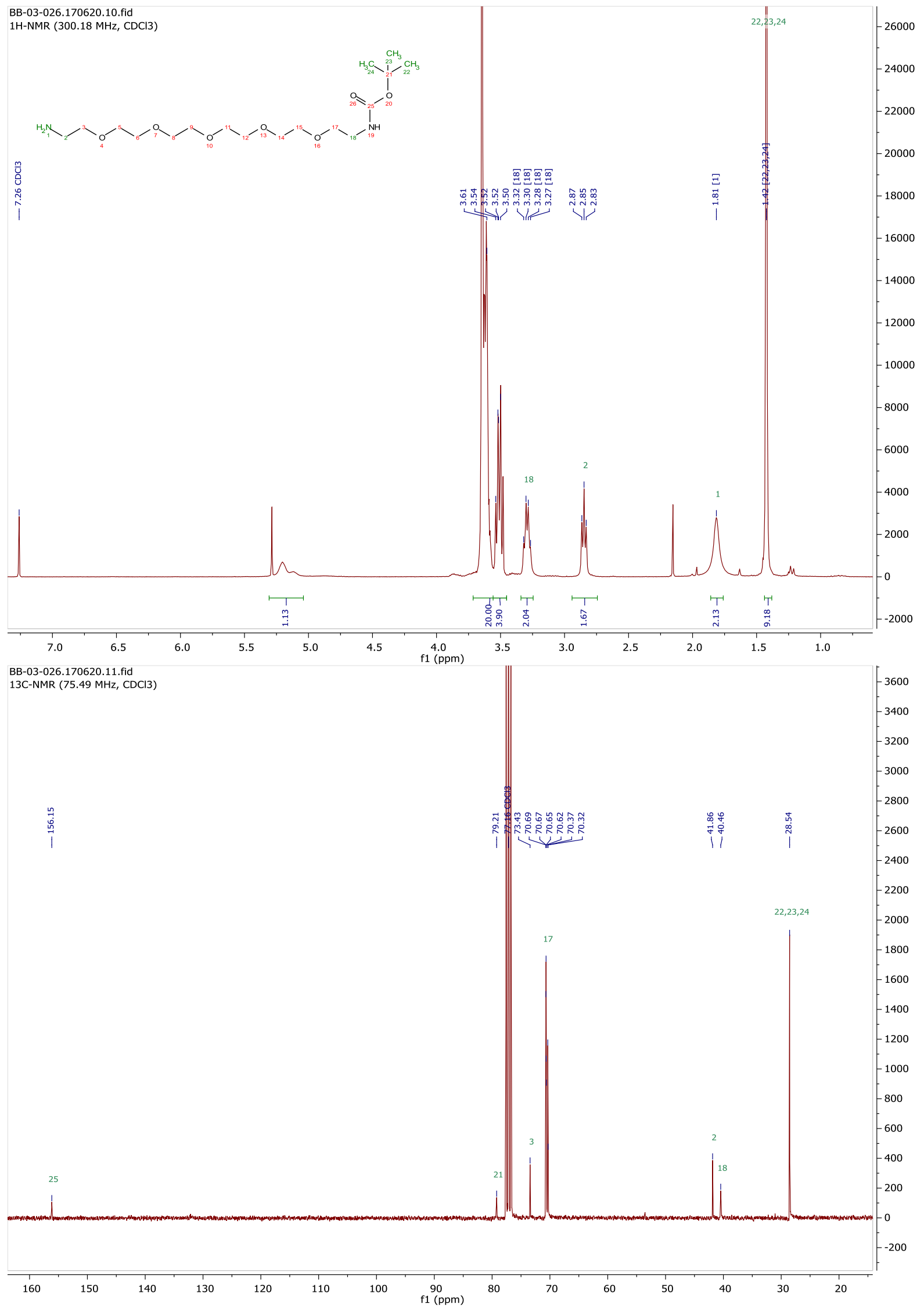


DOTA $\mathbf{N - 1 , 7 - b i s ( b e n z y l ~ e s t e r ) ~ ( 1 ) ~}$

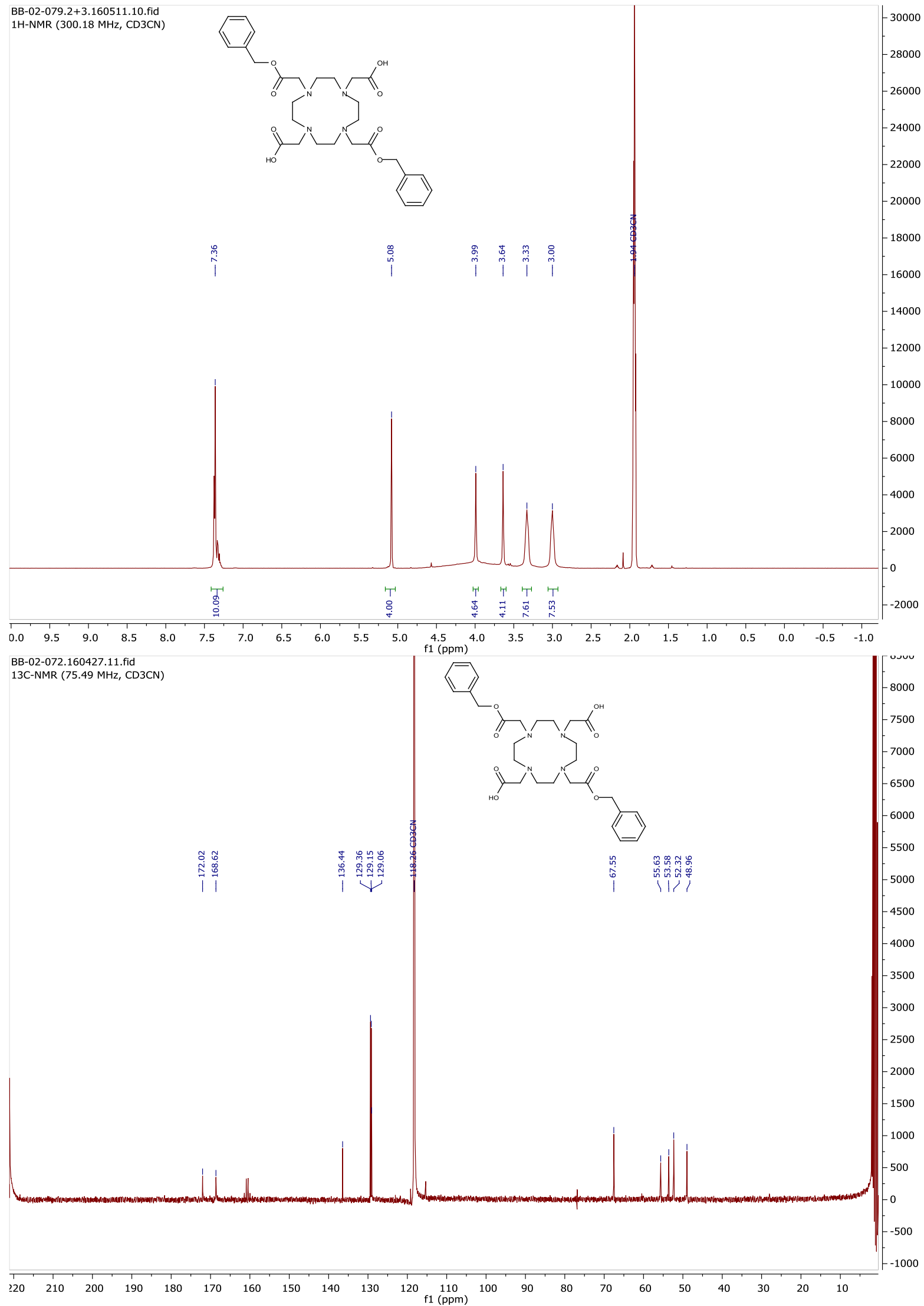




\section{Bis-(PEGNHBoc)-dibenzyl DOTAM (S21)}

BB-03-027.170705.10.fid 1H-NMR (300.18 MHz, CDCl3)
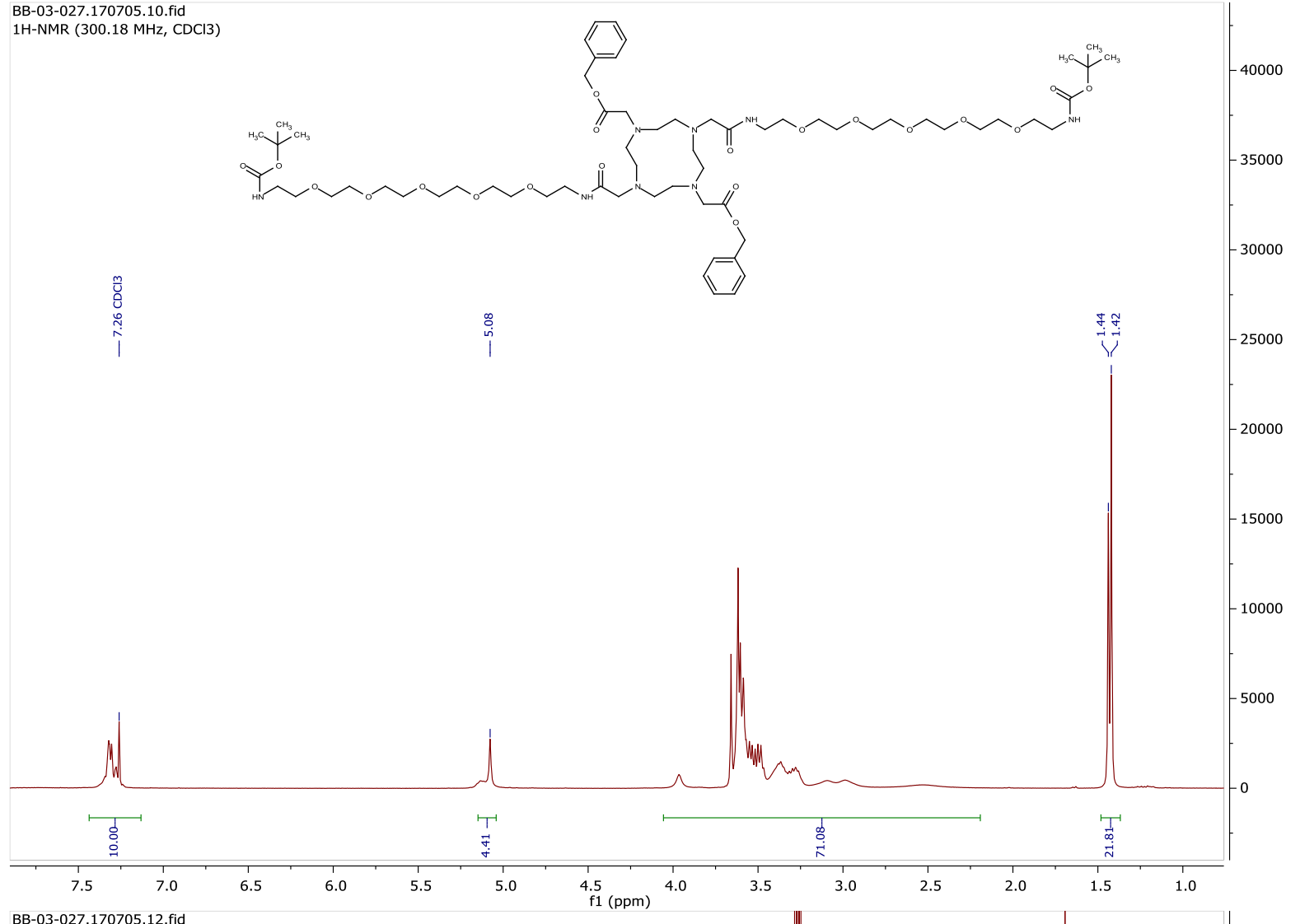

BB-03-027.170705.12 fid 13C-NMR (75.49 MHz, CDCl3)
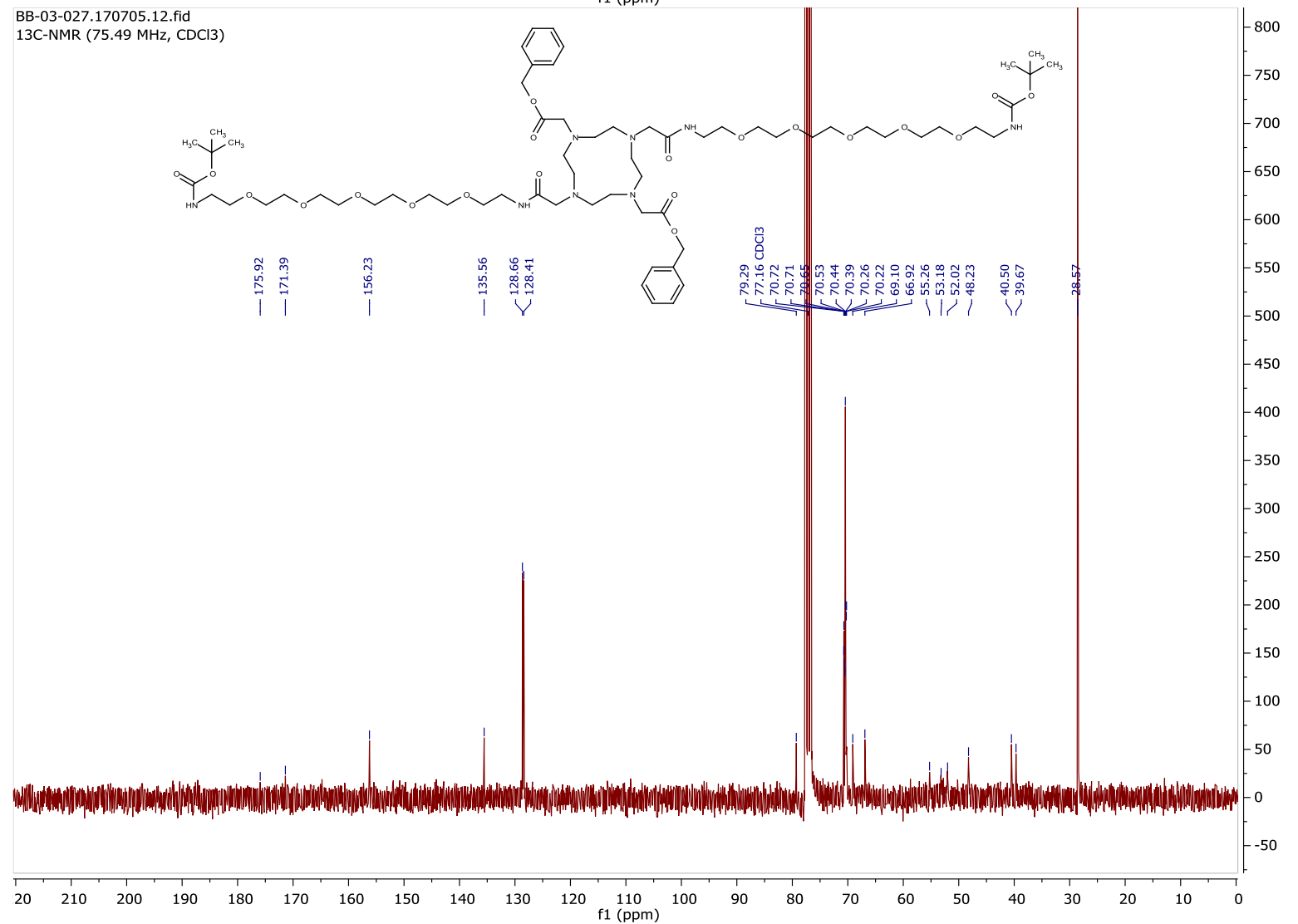

20000

15000

10000

$-5000$ 


\section{Bis-(PEGNHBoc) DOTAM (2)}

BB-03-029.170705.2.10.fid 1H-NMR (300.18 MHz, CD3CN)

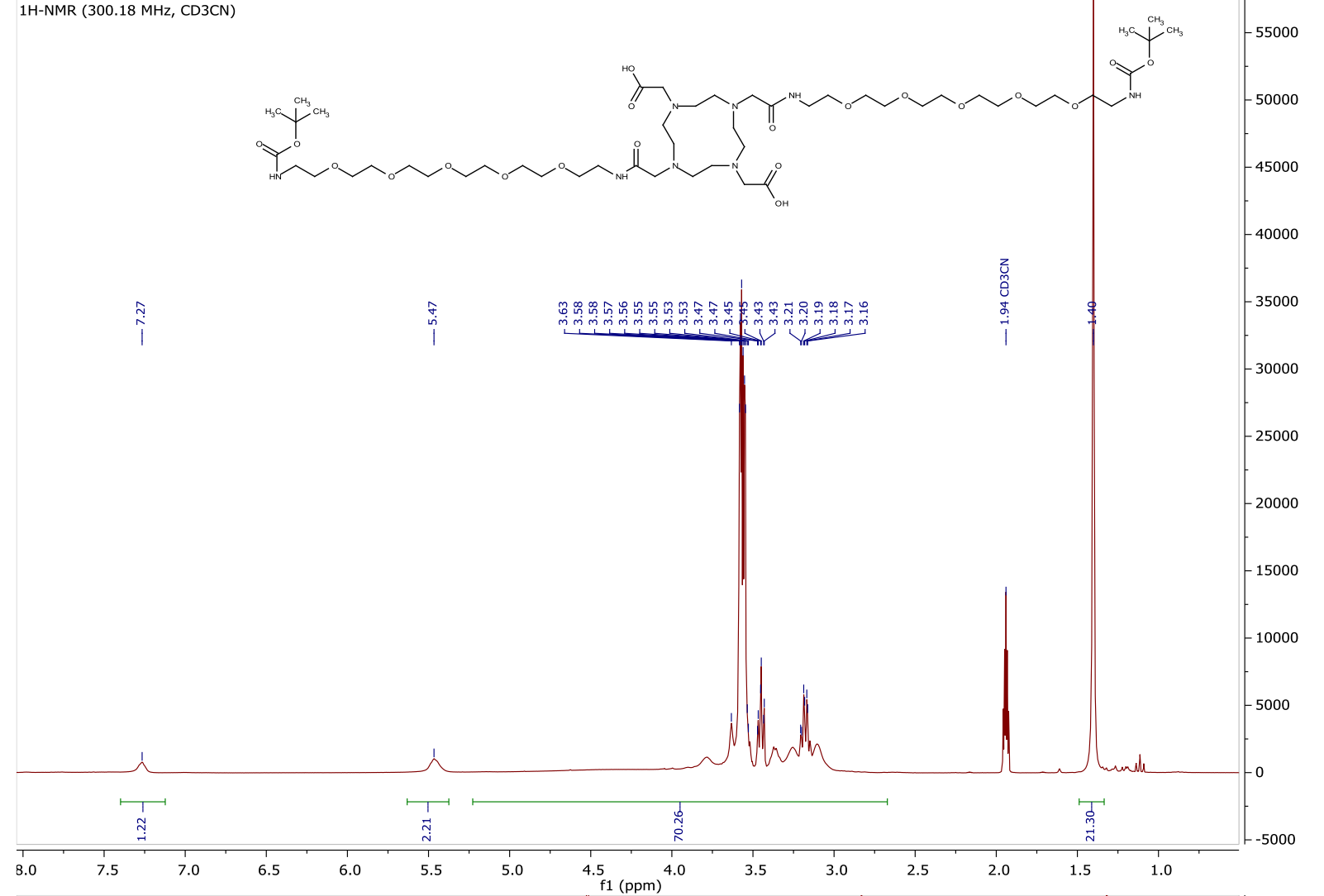

BB-03-029.170705.2.11.fid 13C-NMR (75.49 MHz, CD3CN)

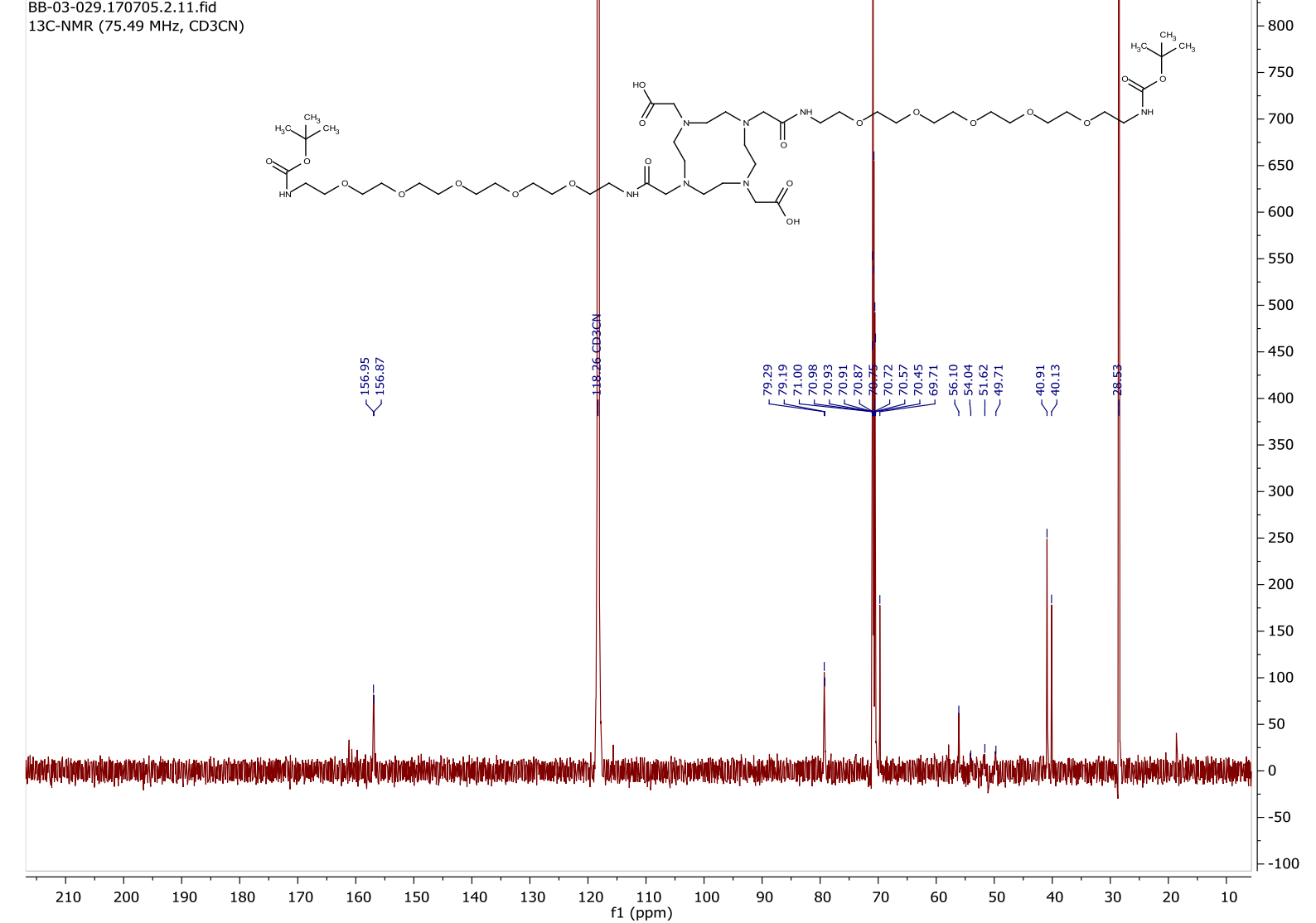




\section{Bis-(PEGNHBoc)-Cy3 DOTAM (3)}

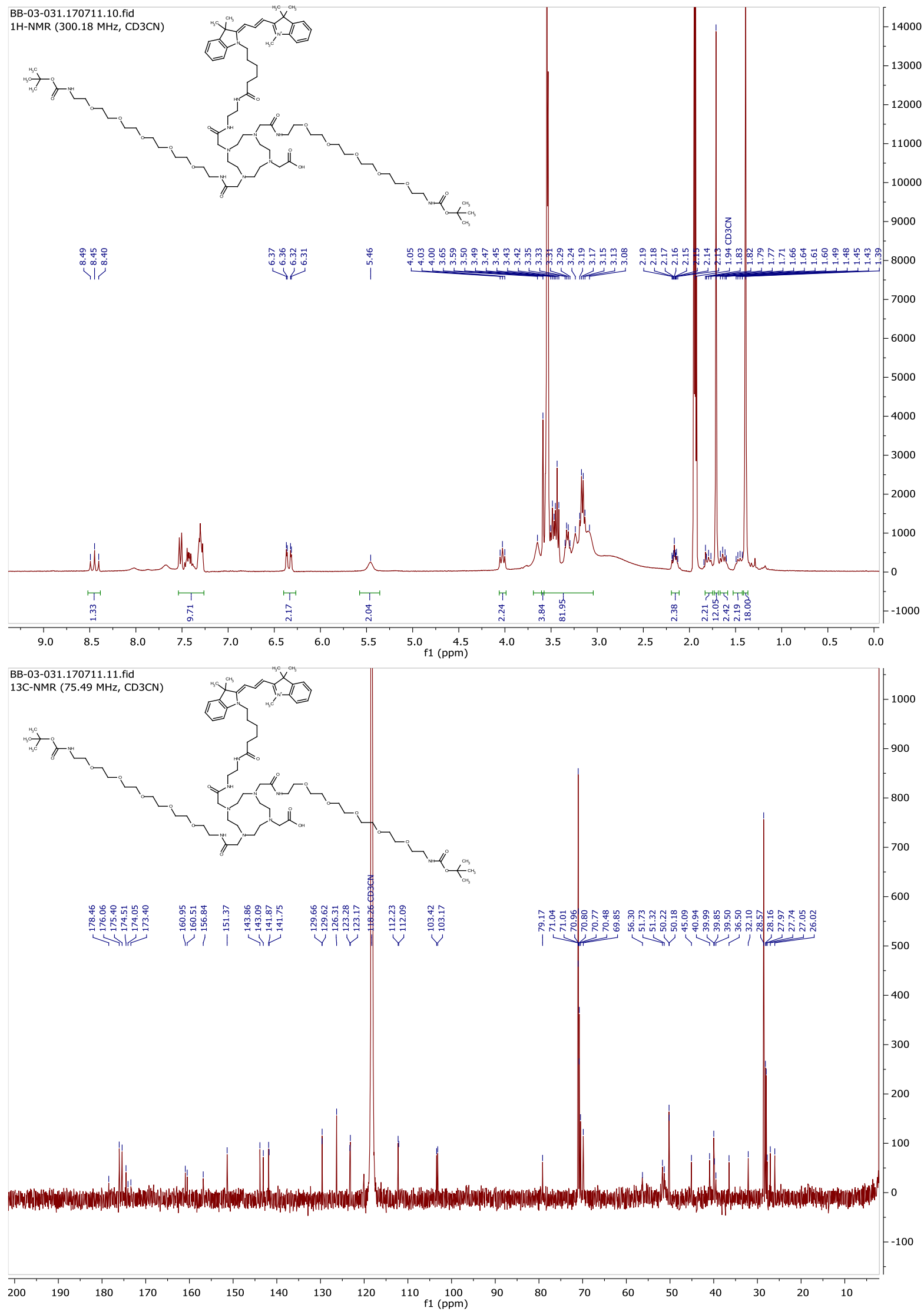


Bis-(PEGNHBoc)-Cy3 DOTAM (3)

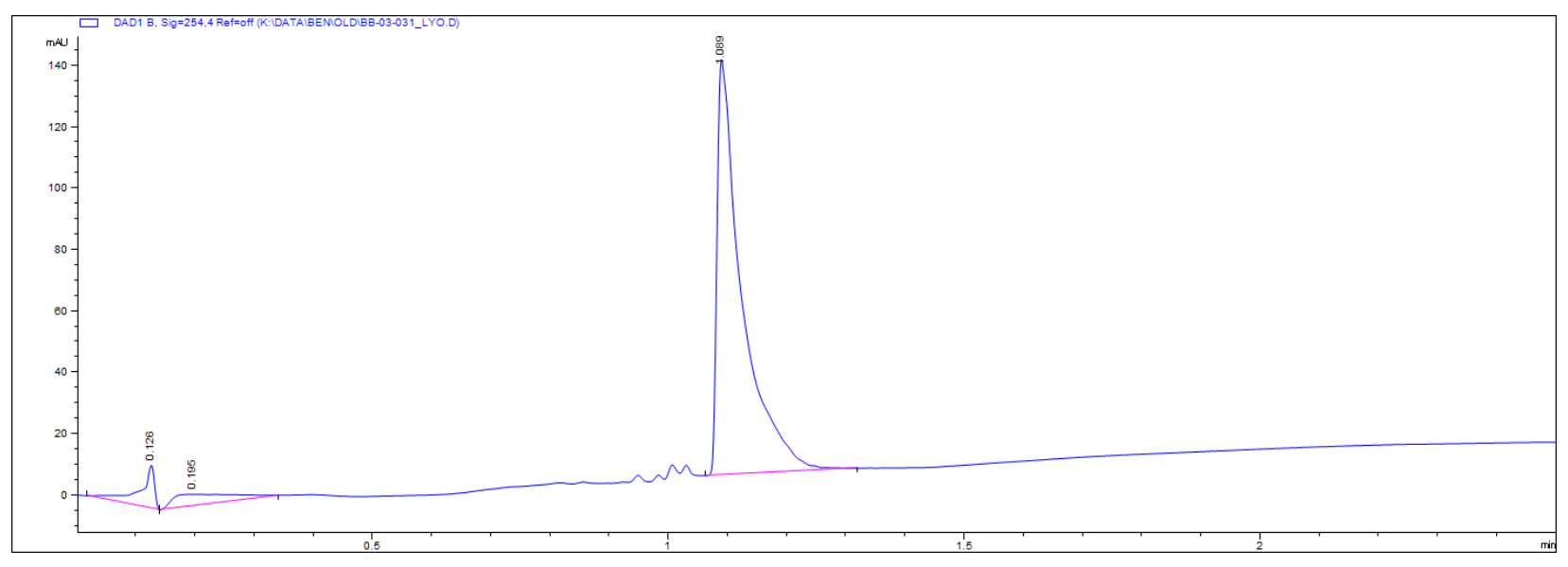

Bis-(PEGNH ${ }_{2}$-Cy3-CatSseq-BHQ-2 DOTAM (BB-03-035) (4)

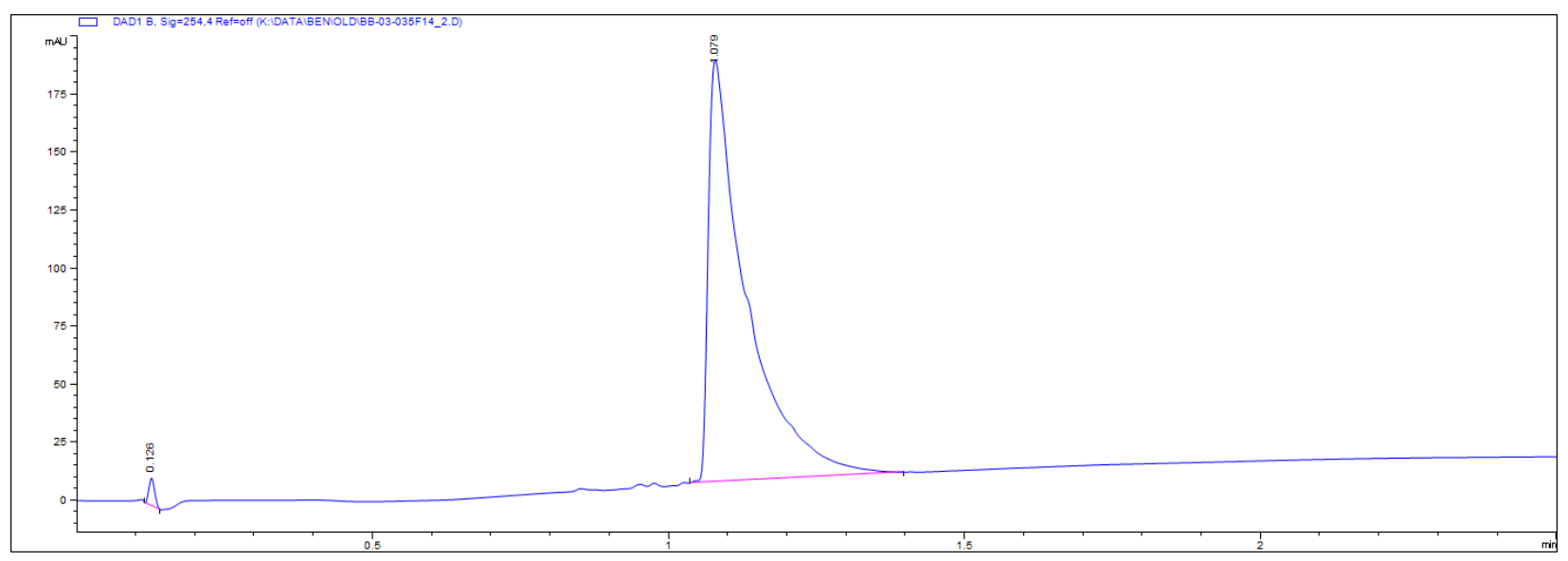


Cy3/BHQ-2 CatS activatable control probe 6

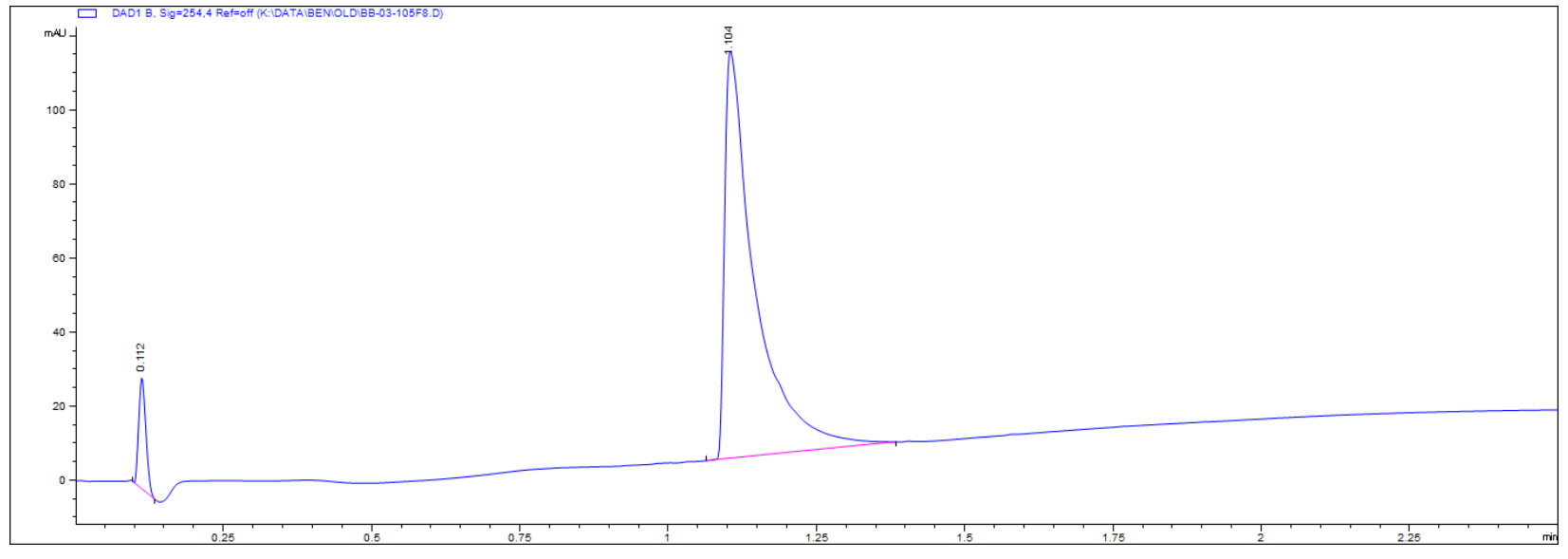

TIC trace with mass peaks at $t=1.14$

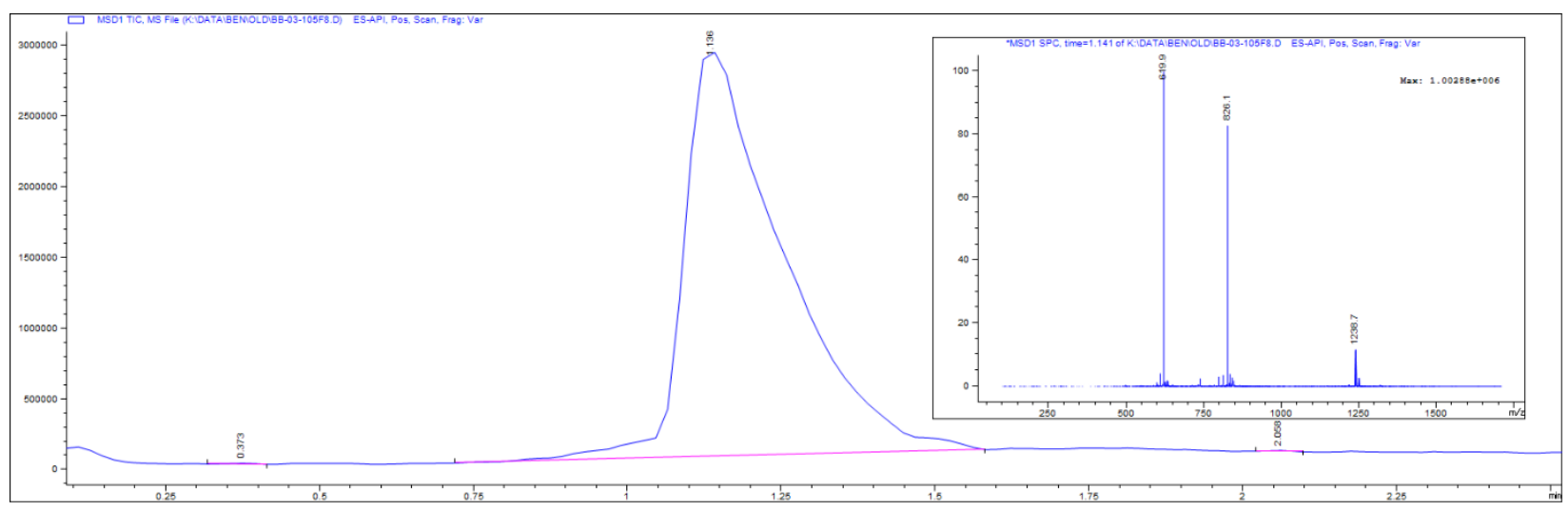




\section{TOF probe 6}

Qualitative Compound Report

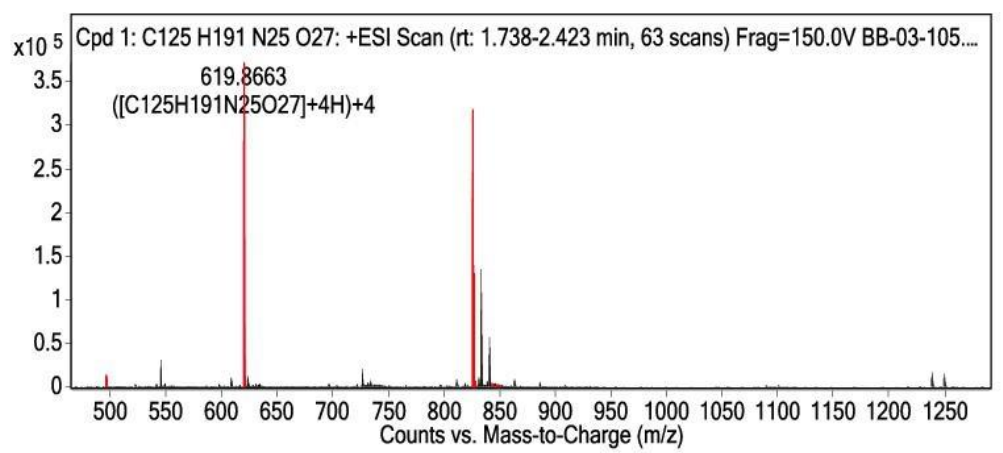

MS Spectrum Peak List

\begin{tabular}{|c|c|c|c|c|c|c|}
\hline$m / z$ & Calc $\mathrm{m} / \mathbf{z}$ & Diff(ppm) & $z$ & Abund & Formula & Ion \\
\hline 495.8935 & \begin{tabular}{|l|}
495.8941 \\
\end{tabular} & 1.22 & 5 & 2119.12 & $\mathrm{C} 125 \mathrm{H} 191 \mathrm{~N} 25 \mathrm{O} 27$ & $(\mathrm{M}+5 \mathrm{H})+5$ \\
\hline 496.094 & 496.0947 & 1.37 & 5 & 3195.13 & C125H191N25027 & $(\mathrm{M}+5 \mathrm{H})+5$ \\
\hline 496.2963 & 496.2953 & -1.98 & 5 & 9254.78 & $\mathrm{C} 125 \mathrm{H} 191 \mathrm{~N} 25027$ & $(M+5 H)+5$ \\
\hline 496.4971 & 496.4959 & -2.44 & 5 & 11788.54 & C125H191N25O27 & $(M+5 H)+5$ \\
\hline 496.6974 & 496.6964 & -2.07 & 5 & 8655.65 & C125H191N25O27 & $(M+5 H)+5$ \\
\hline 496.8981 & 496.897 & -2.19 & 5 & 4411.98 & C125H191N25027 & $(\mathrm{M}+5 \mathrm{H})+5$ \\
\hline 497.0987 & 497.0975 & -2.38 & 5 & 1795.87 & $\mathrm{C} 125 \mathrm{H} 191 \mathrm{~N} 25 \mathrm{O} 27$ & $(\mathrm{M}+5 \mathrm{H})+5$ \\
\hline 619.8663 & 619.8666 & 0.49 & 4 & 371668.69 & $\mathrm{C} 125 \mathrm{H} 191 \mathrm{~N} 25 \mathrm{O} 27$ & $(M+4 H)+4$ \\
\hline 620.1165 & 620.1173 & 1.3 & 4 & 313142.94 & C125H191N25027 & $(M+4 H)+4$ \\
\hline 620.3674 & 620.368 & 1.03 & 4 & 185573.39 & C125H191N25O27 & $(M+4 H)+4$ \\
\hline 620.6178 & 620.6187 & 1.44 & 4 & 88005.53 & C125H191N25027 & $(M+4 H)+4$ \\
\hline 620.8683 & 620.8694 & 1.7 & 4 & 34996.51 & $\mathrm{C} 125 \mathrm{H} 191 \mathrm{~N} 25027$ & $(M+4 H)+4$ \\
\hline 621.1189 & 621.1201 & 1.83 & 4 & 12209.97 & $\mathrm{C} 125 \mathrm{H} 191 \mathrm{~N} 25027$ & $(M+4 H)+4$ \\
\hline 621.3701 & 621.3707 & 1.01 & 4 & 4244.83 & $\mathrm{C} 125 \mathrm{H} 191 \mathrm{~N} 25027$ & $(M+4 H)+4$ \\
\hline 621.6178 & 621.6214 & 5.79 & 4 & 1634.57 & C125H191N25O27 & $(M+4 H)+4$ \\
\hline 636.5976 & 636.6424 & 70.32 & 4 & 581.23 & C125H191N25O27 & $(\mathrm{M}+4(\mathrm{NH} 4))+4$ \\
\hline 636.8508 & 636.8931 & 66.4 & 4 & 377.12 & $\mathrm{C} 125 \mathrm{H} 191 \mathrm{~N} 25 \mathrm{O} 27$ & $(\mathrm{M}+4(\mathrm{NH} 4))+4$ \\
\hline 637.1053 & 637.1438 & 60.5 & 4 & 287.36 & C125H191N25O27 & $(\mathrm{M}+4(\mathrm{NH} 4))+4$ \\
\hline 637.3655 & 637.3945 & 45.59 & 4 & 502.89 & $\mathrm{C} 125 \mathrm{H} 191 \mathrm{~N} 25 \mathrm{O} 27$ & $(\mathrm{M}+4(\mathrm{NH} 4))+4$ \\
\hline 637.6172 & 637.6452 & 43.91 & 4 & 561.84 & C125H191N25027 & $(\mathrm{M}+4(\mathrm{NH} 4))+4$ \\
\hline 637.868 & 637.8959 & 43.68 & 4 & 535.34 & C125H191N25O27 & $(\mathrm{M}+4(\mathrm{NH} 4))+4$ \\
\hline 641.6249 & 641.5978 & -42.25 & 4 & 219.19 & $\mathrm{C} 125 \mathrm{H} 191 \mathrm{~N} 25 \mathrm{O} 27$ & $(\mathrm{M}+4 \mathrm{Na})+4$ \\
\hline 641.8727 & 641.8485 & -37.62 & 4 & 168.38 & $\mathrm{C} 125 \mathrm{H} 191 \mathrm{~N} 25 \mathrm{O} 27$ & $(\mathrm{M}+4 \mathrm{Na})+4$ \\
\hline 642.1231 & 642.0992 & -37.17 & 4 & 86.38 & $\mathrm{C} 125 \mathrm{H} 191 \mathrm{~N} 25027$ & $(M+4 N a)+4$ \\
\hline 642.3775 & 642.35 & -42.83 & 4 & 66.1 & C125H191N25O27 & $(M+4 N a)+4$ \\
\hline 642.6201 & 642.6007 & -30.26 & 4 & 26.74 & $\mathrm{C} 125 \mathrm{H} 191 \mathrm{~N} 25 \mathrm{O} 27$ & $(\mathrm{M}+4 \mathrm{Na})+4$ \\
\hline 825.8205 & 825.8187 & -2.27 & 3 & 200439.22 & $\mathrm{C} 125 \mathrm{H} 191 \mathrm{~N} 25 \mathrm{O} 27$ & $(\mathrm{M}+3 \mathrm{H})+3$ \\
\hline 826.1549 & 826.153 & -2.32 & 3 & 308342.84 & $\mathrm{C} 125 \mathrm{H} 191 \mathrm{~N} 25027$ & $(M+3 H)+3$ \\
\hline 826.4894 & 826.4873 & -2.58 & 3 & 248770.36 & $\mathrm{C} 125 \mathrm{H} 191 \mathrm{~N} 25027$ & $(M+3 H)+3$ \\
\hline 826.824 & 826.8216 & -2.92 & 3 & 139699.16 & $\mathrm{C} 125 \mathrm{H} 191 \mathrm{~N} 25 \mathrm{O} 27$ & $(\mathrm{M}+3 \mathrm{H})+3$ \\
\hline 827.1581 & 827.1559 & -2.66 & 3 & 61127.96 & C125H191N25O27 & $(\mathrm{M}+3 \mathrm{H})+3$ \\
\hline 827.4917 & 827.4901 & -1.88 & 3 & 22181.85 & $\mathrm{C} 125 \mathrm{H} 191 \mathrm{~N} 25 \mathrm{O} 27$ & $(\mathrm{M}+3 \mathrm{H})+3$ \\
\hline 827.8249 & 827.8243 & -0.73 & 3 & 7359.39 & $\mathrm{C} 125 \mathrm{H} 191 \mathrm{~N} 25 \mathrm{O} 27$ & $(M+3 H)+3$ \\
\hline 828.1569 & 828.1586 & 1.99 & 3 & 2600.87 & $\mathrm{C} 125 \mathrm{H} 191 \mathrm{~N} 25027$ & $(M+3 H)+3$ \\
\hline 843.1448 & 843.1795 & 41.23 & 3 & 902.72 & C125H191N25O27 & $(\mathrm{M}+3(\mathrm{NH} 4))+3$ \\
\hline 843.4734 & 843.5138 & 47.89 & 3 & 839.62 & C125H191N25027 & $(\mathrm{M}+3(\mathrm{NH} 4))+3$ \\
\hline 843.7979 & 843.8481 & 59.54 & 3 & 2149.36 & C125H191N25O27 & $(\mathrm{M}+3(\mathrm{NH} 4))+3$ \\
\hline 844.1308 & 844.1824 & 61.02 & 3 & 2754.09 & C125H191N25O27 & $(\mathrm{M}+3(\mathrm{NH} 4))+3$ \\
\hline 844.4666 & 844.5166 & 59.23 & 3 & 2755.44 & $\mathrm{C} 125 \mathrm{H} 191 \mathrm{~N} 25027$ & $(\mathrm{M}+3(\mathrm{NH} 4))+3$ \\
\hline 847.8072 & 847.8006 & -7.78 & 3 & 1726.38 & C125H191N25O27 & $(\mathrm{M}+3 \mathrm{Na})+3$ \\
\hline 848.1432 & 848.1349 & -9.69 & 3 & 2016.65 & C125H191N25027 & $(\mathrm{M}+3 \mathrm{Na})+3$ \\
\hline 848.4761 & 848.4692 & -8.14 & 3 & 1875.38 & C125H191N25O27 & $(\mathrm{M}+3 \mathrm{Na})+3$ \\
\hline 848.807 & 848.8035 & -4.05 & 3 & 1488.76 & $\mathrm{C} 125 \mathrm{H} 191 \mathrm{~N} 25027$ & $(\mathrm{M}+3 \mathrm{Na})+3$ \\
\hline 1238.2232 & 1238.2243 & 0.91 & 2 & 10415.28 & $\mathrm{C} 125 \mathrm{H} 191 \mathrm{~N} 25027$ & $(\mathrm{M}+2 \mathrm{H})+2$ \\
\hline 1238.725 & 1238.7258 & 0.68 & 2 & 16416.47 & C125H191N25027 & $(\mathrm{M}+2 \mathrm{H})+2$ \\
\hline 1239.226 & 1239.2273 & 1.05 & 2 & 12843.48 & C125H191N25O27 & $(\mathrm{M}+2 \mathrm{H})+2$ \\
\hline
\end{tabular}


Qualitative Compound Report

\begin{tabular}{|r|r|r|r|r|l|l|}
\hline $\boldsymbol{m} / \boldsymbol{z}$ & Calc $\boldsymbol{m} / \boldsymbol{z}$ & \multicolumn{1}{l|}{ Diff(ppm) } & $\mathbf{z}$ & \multicolumn{1}{l}{ Abund } & Formula & Ion \\
\hline 1239.7272 & 1239.7287 & 1.28 & 2 & 6782.31 & $\mathrm{C} 125 \mathrm{H} 191 \mathrm{~N} 25027$ & $(\mathrm{M}+2 \mathrm{H})+2$ \\
\hline 1240.2284 & 1240.2301 & 1.38 & 2 & 2821.06 & $\mathrm{C} 125 \mathrm{H} 191 \mathrm{~N} 25027$ & $(\mathrm{M}+2 \mathrm{H})+2$ \\
\hline 1240.7298 & 1240.7315 & 1.4 & 2 & 1036.39 & $\mathrm{C} 125 \mathrm{H} 191 \mathrm{~N} 25027$ & $(\mathrm{M}+2 \mathrm{H})+2$ \\
\hline 1241.2305 & 1241.2329 & 1.9 & 2 & 328.73 & $\mathrm{C} 125 \mathrm{H} 191 \mathrm{~N} 25027$ & $(\mathrm{M}+2 \mathrm{H})+2$ \\
\hline 1241.732 & 1241.7342 & 1.75 & 2 & 45.96 & $\mathrm{C} 125 \mathrm{H} 191 \mathrm{~N} 25027$ & $(\mathrm{M}+2 \mathrm{H})+2$ \\
\hline 1260.7219 & 1260.7078 & -11.21 & 2 & 94.19 & $\mathrm{C} 125 \mathrm{H} 191 \mathrm{~N} 25027$ & $(\mathrm{M}+2 \mathrm{Na})+2$ \\
\hline 1261.2208 & 1261.2093 & -9.19 & 2 & 92 & $\mathrm{C} 125 \mathrm{H} 191 \mathrm{~N} 25027$ & $(\mathrm{M}+2 \mathrm{Na})+2$ \\
\hline 1261.7194 & 1261.7107 & -6.88 & 2 & 39.21 & $\mathrm{C} 125 \mathrm{H} 191 \mathrm{~N} 25027$ & $(\mathrm{M}+2 \mathrm{Na})+2$ \\
\hline
\end{tabular}

-- End Of Report -- 
Bis-(PEGNHsucc)-Cy3-CatSseq-BHQ-2 DOTAM (S22)

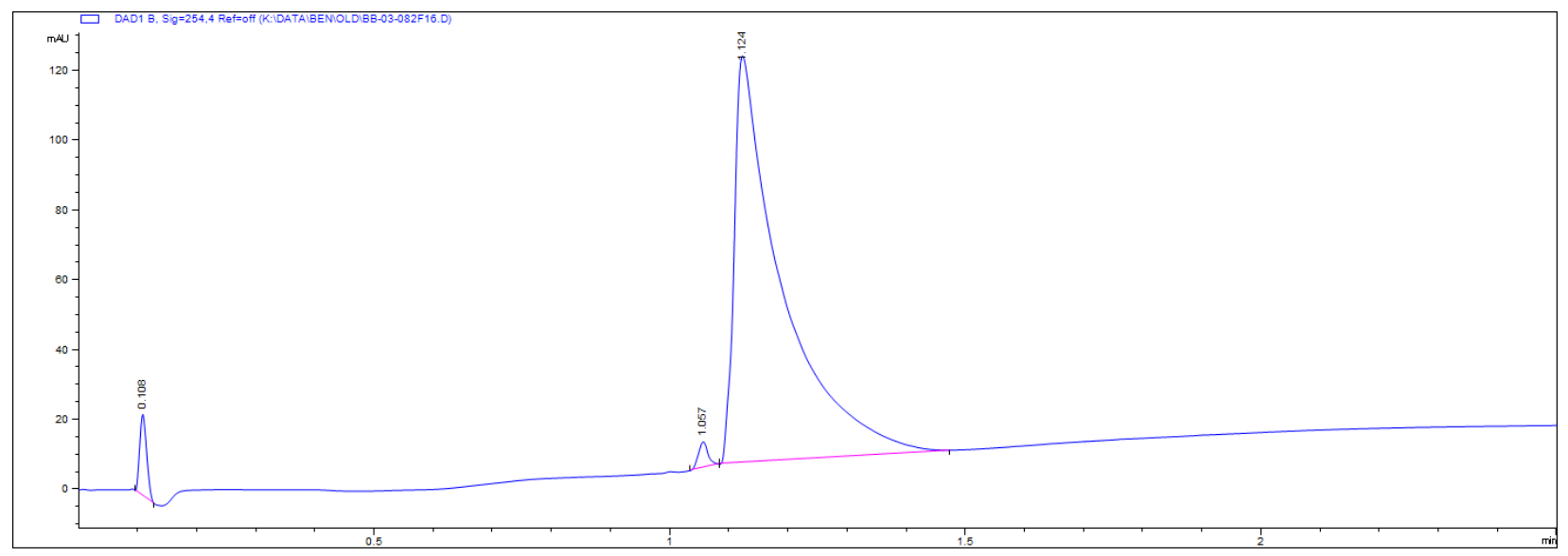

Bis-(cRGDfK)-Cy3/BHQ-2 CatS activatable probe 5

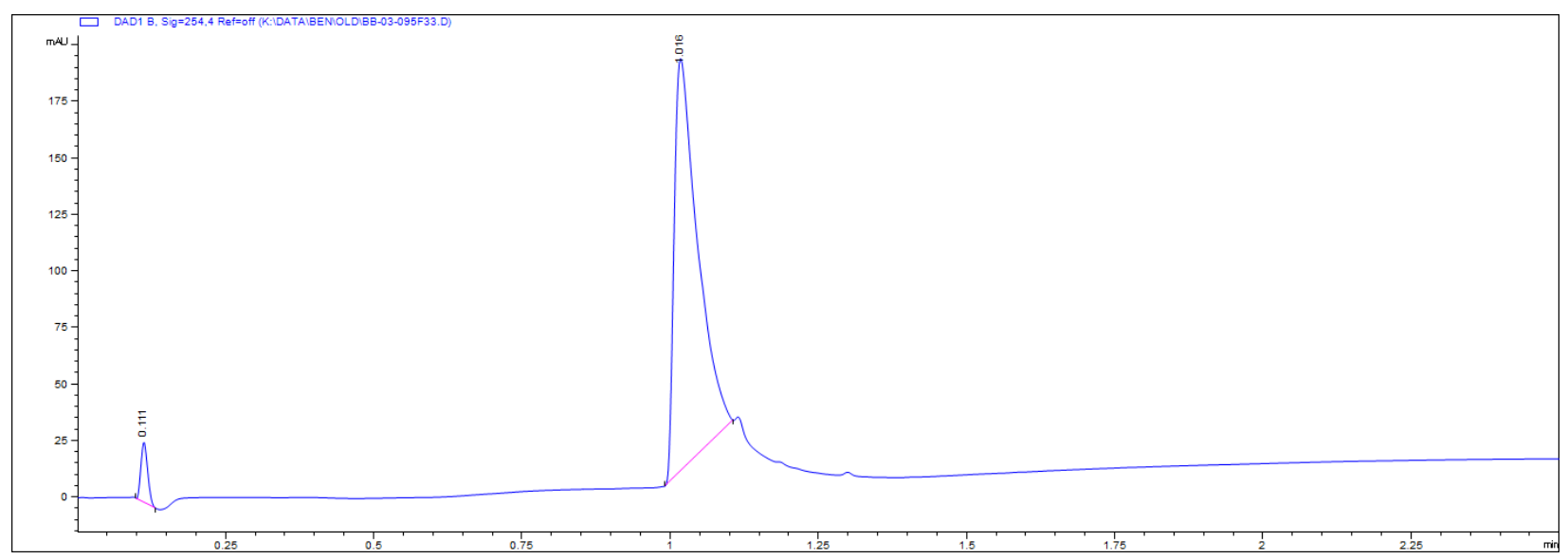

TIC trace with mass peaks at $t=1.08$

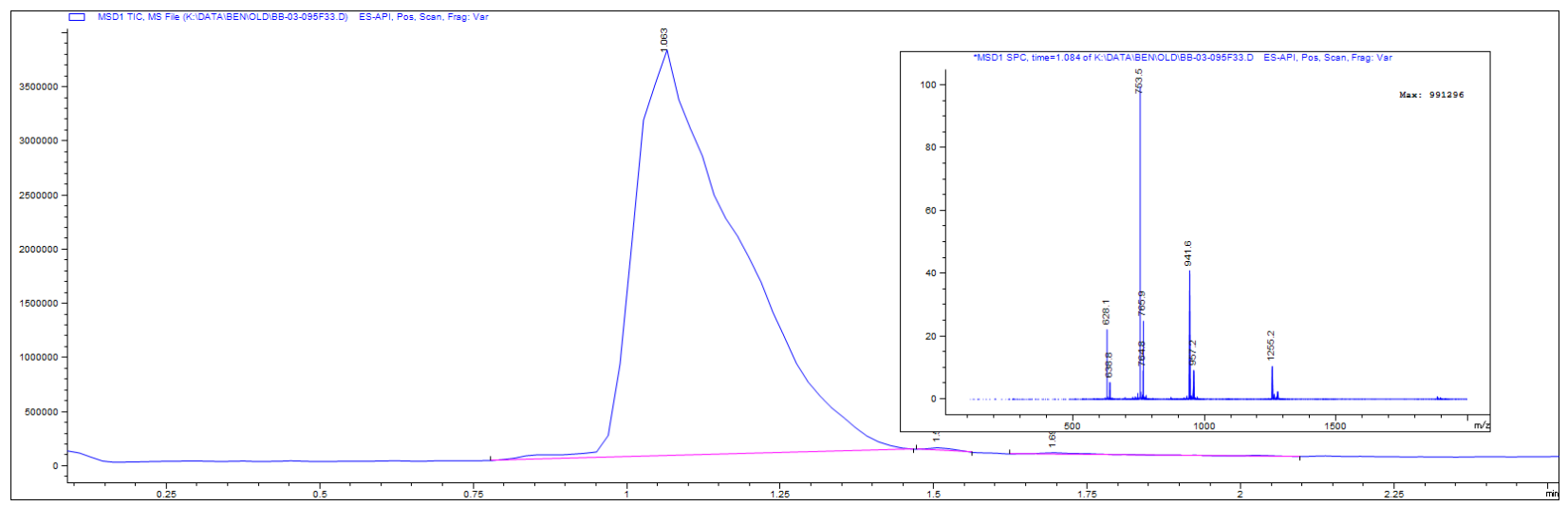

S46 
TOF probe 5

\section{Qualitative Compound Report}

\begin{tabular}{|c|c|c|c|}
\hline 1277.6731 & & $\begin{array}{l}989.13 \\
\mathrm{C} 183 \mathrm{H} 273 \mathrm{~N} 43043\end{array}$ & $(\mathrm{M}+3 \mathrm{~N} \mathrm{Na})+3$ \\
\hline 1278.007 & 3 & $632.61 \mathrm{C1} 183 \mathrm{H} 273 \mathrm{~N} 43 \mathrm{O} 43$ & $(\mathrm{M}+3 \mathrm{Na})+3$ \\
\hline 1278.342 & 3 & 344.81 C183H273N43043 & $(M+3 N a)+3$ \\
\hline 1278.6771 & 3 & $134.77 \mathrm{C183 \textrm {H } 2 7 3 \mathrm { N } 4 3 \mathrm { O } 4 3}$ & $(\mathrm{M}+3 \mathrm{Na})+3$ \\
\hline 1882.0254 & 2 & $71.15 \mathrm{Cl} 183 \mathrm{H} 273 \mathrm{~N} 43043$ & $(M+2 H)+2$ \\
\hline 1882.525 & $\frac{2}{2}$ & $\begin{array}{lll}90.01 \mathrm{C} 183 \mathrm{H} 273 \mathrm{~N} 43043 \\
\end{array}$ & $(\mathrm{M}+2 \mathrm{H})+2$ \\
\hline $1883.02 \approx 8$ & 2 & 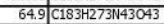 & $(M+2 H)+2$ \\
\hline
\end{tabular}

MS Spectrum

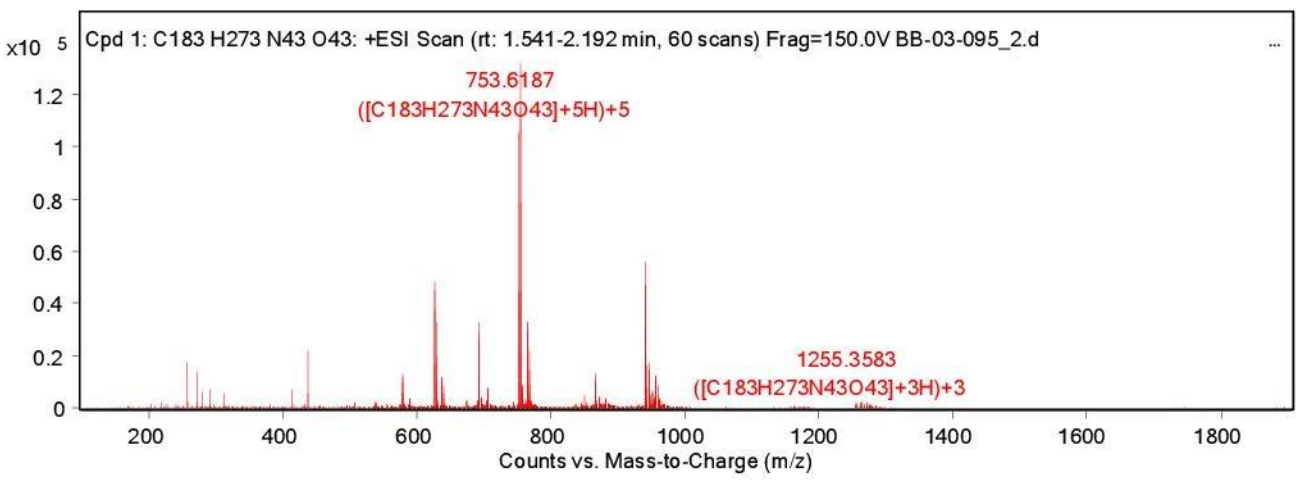

MS Zoomed Spectrum
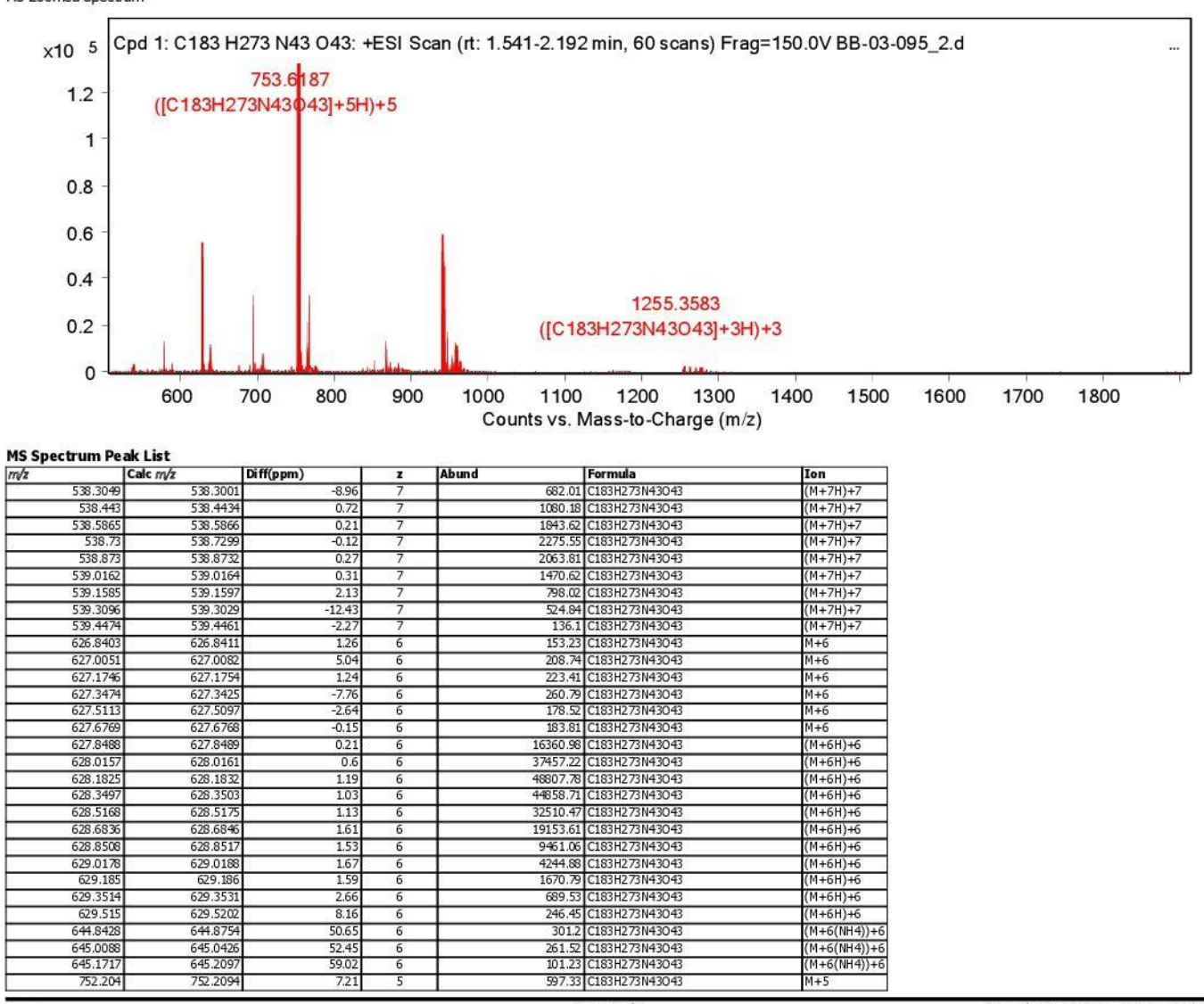

Page 4 of 5 


\section{Qualitative Compound Report}

\begin{tabular}{|c|c|c|c|c|c|c|}
\hline 752.4072 & 752.41 & 3.7 & 5 & 617.66 & $\mathrm{C} 183 \mathrm{H} 273 \mathrm{~N} 43043$ & $M+5$ \\
\hline 752.6099 & 752.6106 & 2.24 & 5 & 524.8 & $\mathrm{Cl} 183 \mathrm{H} 273 \mathrm{~N} 43 \mathrm{O} 43$ & $M+5$ \\
\hline 752.8073 & 752.8111 & 5.09 & 5 & 426.6 & $\mathrm{C1} 183 \mathrm{H} 273 \mathrm{~N} 43 \mathrm{O} 43$ & $M+5$ \\
\hline 753.0116 & 753.0117 & 0.1 & 5 & 460.21 & $\mathrm{C1} 183 \mathrm{H} 273 \mathrm{~N} 43 \mathrm{O} 43$ & $M+5$ \\
\hline 753.217 & 753.217 & -0.74 & 5 & 44485.8 & $\mathrm{C} 183 \mathrm{H} 273 \mathrm{~N} 43043$ & $(M+5 \mathrm{H})+5$ \\
\hline 753.4186 & 753.4178 & -1.01 & 5 & 105822.16 & $\mathrm{C1} 183 \mathrm{H} 273 \mathrm{~N} 43043$ & $(M+5 H)+5$ \\
\hline 753.6187 & 753.6184 & -0.44 & 5 & 132399.7 & $\mathrm{C1} 183 \mathrm{H} \mathrm{H} 273 \mathrm{~N} / 43043$ & $(M+5 H)+5$ \\
\hline 753.819 & 753.819 & -0.1 & 5 & 115473.73 & $1183 \mathrm{H} 273 \mathrm{~N} 43043$ & $(M+5 H)+5$ \\
\hline 754.0195 & 754.0195 & 0.04 & 5 & 78249.24 & $\mathrm{C183 \textrm {H } 2 7 3 \mathrm { N } 4 3 0 4 3}$ & $(M+5 H)+5$ \\
\hline 754.2196 & 754.2201 & 0.59 & 5 & $44323.7 \pi$ & $\mathrm{C} 183 \mathrm{H} 2 \mathrm{T3} \mathrm{N} 43 \mathrm{O} 43$ & $(M+5 H)+5$ \\
\hline 754.4202 & 754.4206 & 0.58 & 5 & 21131.29 & $\mathrm{C} 183 \mathrm{H} 273 \mathrm{~N} 43 \mathrm{O} 43$ & $(M+5 H)+5$ \\
\hline 754.6202 & 754.6212 & 1.31 & 5 & 8935.35 & $\mathrm{C1} 183 \mathrm{H} 273 \mathrm{~N} 43 \mathrm{O} 43$ & $(M+5 H)+5$ \\
\hline 754.82 & 754.8217 & 2.19 & 5 & 3554.23 & $\mathrm{C1} 83 \mathrm{H} 273 \mathrm{~N} 43 \mathrm{O} 43$ & $(M+5 H)+5$ \\
\hline 755.019 & 755.0222 & 4.25 & 5 & 1502.35 & $\mathrm{C1} 183 \mathrm{H} 273 \mathrm{~N} 43043$ & $(M+5 H)+5$ \\
\hline 755.2129 & 755.2228 & 13.01 & 5 & 778.52 & $\mathrm{C1} 183 \mathrm{H} 273 \mathrm{~N} 43 \mathrm{O} 43$ & $(M+5 H)+5$ \\
\hline 755.4074 & 755.4233 & 20.98 & 5 & 590.91 & $\mathrm{C1} 183 \mathrm{H} 273 \mathrm{~N} 43043$ & $(M+5 H)+5$ \\
\hline 770.403 & 70.4444 & 53.66 & 5 & 1093.1 & $\mathrm{C1} 183 \mathrm{H} \mathrm{H} 273 \mathrm{~N} / 43043$ & $(\mathrm{M}+5(\mathrm{MH} 4))+5$ \\
\hline 70.6079 & 700.6449 & 48.06 & 5 & 741.61 & $\mathrm{C183} \mathrm{H} 273 \mathrm{~N} / 43043$ & $(M+5(\mathrm{MH} / 4))+5$ \\
\hline 770.8146 & 770.8455 & 40.02 & 5 & 635.12 & $\mathrm{C1} 183 \mathrm{H} 273 \mathrm{~N} 43043$ & $(M+5(\mathrm{NH} 4))+5$ \\
\hline 771.0116 & 771.046 & 44.64 & 5 & 555.62 & $\mathrm{C} 183 \mathrm{H} 273 \mathrm{~N} 43 \mathrm{O} 043$ & $(M+5(\mathrm{NH} 4))+5$ \\
\hline 771.2135 & 771.2466 & 42.89 & 5 & 497.02 & $\mathrm{C} 183 \mathrm{H} 273 \mathrm{~N} 43 \mathrm{O} 43$ & $(\mathrm{M}+5(\mathrm{NH} 4))+5$ \\
\hline 775.2019 & 775.1992 & -3.55 & 5 & 735.05 & $\mathrm{C1} 183 \mathrm{H} 273 \mathrm{~N} 43 \mathrm{O} 43$ & $f(M+5 \mathrm{Na})+5$ \\
\hline 775.4032 & 775.3998 & -4.46 & 5 & 788.94 & $\mathrm{C1} 183 \mathrm{H} 273 \mathrm{~N} 43043$ & $(\mathrm{M}+5 \mathrm{Na})+5$ \\
\hline 775.6039 & 775.6003 & -4.63 & 5 & 795.57 & $\mathrm{C1} 183 \mathrm{H} 273 \mathrm{~N} 43043$ & $(\mathrm{M}+5 \mathrm{Na})+5$ \\
\hline 775.8056 & 775.8009 & -6.02 & 5 & 735.38 & $\mathrm{C1} 183 \mathrm{H} 273 \mathrm{~N} 43043$ & $(\mathrm{M}+5 \mathrm{Na})+5$ \\
\hline 776.0157 & 776.0015 & -18.36 & 5 & 978.97 & $1183 \mathrm{H} 273 \mathrm{~N} / 43043$ & $(\mathrm{M}+5 \mathrm{Na})+5$ \\
\hline 776.2204 & 776.202 & -23.71 & 5 & 1419.18 & $\mathrm{C1} 183 \mathrm{H} / 273 \mathrm{~N} / 43043$ & $(\mathrm{M}+5 \mathrm{Na})+5$ \\
\hline 776.4227 & 777.4026 & -25.89 & 5 & 1653 & $\mathrm{C1} 183 \mathrm{H} 273 \mathrm{~N} 43043$ & $(\mathrm{M}+5 \mathrm{Na})+5$ \\
\hline 776.6234 & 776.6031 & -26.11 & 5 & 1494.86 & $\mathrm{C1} 183 \mathrm{H} 273 \mathrm{~N} 43043$ & $(\mathrm{M}+5 \mathrm{Na})+5$ \\
\hline 940.2530 & 940.2619 & 8.57 & 4 & 325.71 & $\mathrm{C1} 183 \mathrm{H} 273 \mathrm{~N} 43043$ & MM+4 \\
\hline 940.508 & 940.5126 & 4.94 & 4 & 278.08 & $\mathrm{C} 183 \mathrm{H} 273 \mathrm{~N} 43 \mathrm{O} 43$ & $M+4$ \\
\hline 940.7571 & 940.7633 & 6.69 & 4 & 230.85 & $\mathrm{C1} 183 \mathrm{H} 273 \mathrm{~N} 43 \mathrm{O} 43$ & $M+4$ \\
\hline 941.0101 & 941.0141 & 4.22 & 4 & 217.54 & $\mathrm{C1} 183 \mathrm{H} 273 \mathrm{~N} 43043$ & $M+4$ \\
\hline 941.2707 & 941.2697 & -1.01 & 4 & 18970.7 & $\mathrm{C} 183 \mathrm{H} 273 \mathrm{~N} 43043$ & $(M+4 H)+4$ \\
\hline 941.5219 & 941.5205 & -1.54 & $\frac{7}{4}$ & 45528.5 & C183H273N43043 & $\frac{(M+4+4 H)+4}{(M+4}$ \\
\hline 941.7725 & 941.7712 & -1.36 & 4 & 55937.95 & $\mathrm{C1} 183 \mathrm{H} 273 \mathrm{~N} 43043$ & $(M+4 H)+4$ \\
\hline 942.0228 & 942.0219 & -1.01 & 4 & 47300.7 & $\mathrm{C1} 183 \mathrm{H} / 273 \mathrm{~N} / 4043$ & $(M+4 H)+4$ \\
\hline 942.2735 & 942.2726 & -0.98 & 4 & 30571.06 & $\mathrm{C1} 183 \mathrm{H} 273 \mathrm{~N} / 43043$ & $(M+4 H)+4$ \\
\hline 942.5239 & 942.5233 & -0.72 & 4 & 16121.33 & $\mathrm{C1} 183 \mathrm{H} 273 \mathrm{~N} 43 \mathrm{O}-43$ & $(M+4 H)+4$ \\
\hline 942.7748 & 942.774 & -0.94 & 4 & 7353.80 & $\mathrm{C1} 183 \mathrm{H} 273 \mathrm{~N} 43043$ & $(M+4 H)+4$ \\
\hline 943.024 & 943.0246 & 0.68 & 4 & 3087.43 & $\mathrm{C} 183 \mathrm{H} 273 \mathrm{~N} 43 \mathrm{O} 43$ & $(M+4 H)+4$ \\
\hline 943.2732 & 943.2753 & 2.24 & 4 & 1322.92 & $\mathrm{C1} 183 \mathrm{H} 273 \mathrm{~N} 43 \mathrm{O} 43$ & $(M+4 H)+4$ \\
\hline 943.5177 & 943.526 & 8.75 & 4 & 712.28 & $\mathrm{C1} 183 \mathrm{H} 273 \mathrm{~N} 43043$ & $(M+4 H)+4$ \\
\hline 943.7644 & 943.7766 & 12.93 & 4 & 533.55 & $\mathrm{C1} 183 \mathrm{H} 273 \mathrm{~N} 43043$ & $(M+4 H)+4$ \\
\hline 958.2536 & 958.2963 & 44.48 & 4 & 11109.63 & $\mathrm{C1} 183 \mathrm{H} 273 \mathrm{~N} 43 \mathrm{O} 43$ & $(\mathrm{M}+4(\mathrm{NH} 4))+4$ \\
\hline 958.5048 & 958.547 & 44.05 & 4 & 7944.15 & C183H273N43043 & $(M+4(\mathrm{MH} 4))+4$ \\
\hline 958.7553 & 958.7977 & 44.2 & 4 & 4804.17 & \begin{tabular}{|c|c|}
$183 \mathrm{H} 273 \mathrm{~N} 43043$ \\
\end{tabular} & $(M+4(N H 4))+4$ \\
\hline 959.0068 & 959.0484 & 43.41 & 4 & 2789.68 & $\mathrm{C1} 183 \mathrm{H} 273 \mathrm{~N} / 43043$ & $(M+4(N H 4))+4$ \\
\hline 959.2586 & 959.2991 & 42.25 & 4 & 1640.44 & $\mathrm{C1} 183 \mathrm{H} 273 \mathrm{~N} 43043$ & $(\mathrm{M}+4(\mathrm{NH} 4))+4$ \\
\hline 959.5133 & 959.5498 & 37.99 & 4 & 1110.13 & $\mathrm{C} 183 \mathrm{H} 273 \mathrm{~N} 43043$ & $(\mathrm{M}+4(\mathrm{NH} 4))+4$ \\
\hline 959.7663 & 959.8005 & 35.53 & 4 & 861.8 & $\mathrm{C} 183 \mathrm{H} 273 \mathrm{~N} 43 \mathrm{O} 43$ & $(M+4(N H 4))+4$ \\
\hline 960.0162 & 960.0511 & 36.4 & 4 & 715.84 & $\mathrm{C183} \mathrm{H} 273 \mathrm{~N} 43 \mathrm{O} 43$ & $(\mathrm{M}+4(\mathrm{NH} 4))+4$ \\
\hline 960.2581 & 960.3018 & 45.45 & 4 & 841.62 & $\mathrm{C} 183 \mathrm{H} 273 \mathrm{~N} 43 \mathrm{O} 43$ & $(\mathrm{M}+4(\mathrm{NH} 4))+4$ \\
\hline 963.248 & 963.2517 & 3.81 & 4 & 3458.04 & $\mathrm{C1} 183 \mathrm{H} 273 \mathrm{~N} 43 \mathrm{O} 43$ & $(\mathrm{M}+4 \mathrm{Na})+4$ \\
\hline 963.4992 & 963.5024 & 3.34 & 4 & 3734.01 & $\mathrm{C} 183 \mathrm{H} 273 \mathrm{~N} 43043$ & $(\mathrm{M}+4 \mathrm{Na})+4$ \\
\hline 963.7507 & 963.7531 & 2.55 & 4 & 3330.43 & $\mathrm{C1} 183 \mathrm{H} 273 \mathrm{~N} 43 \mathrm{O} 43$ & $(M+4 N a)+4$ \\
\hline 964.0016 & 964.0038 & 2.28 & 4 & 2434.68 & $1183 \mathrm{H} 273 \mathrm{~N} 43043$ & $(M+4 N a)+4$ \\
\hline 964.2523 & 964.2545 & 2.33 & 4 & 1575.1 & $\mathrm{C} 183 \mathrm{H} 273 \mathrm{~N} / 43043$ & $(M+4 N a)+4$ \\
\hline 964.5034 & 964.5052 & 1.91 & 4 & 984.89 & $\mathrm{C183} 3 \mathrm{H} 273 \mathrm{~N} / 43043$ & $(M+4 N a)+4$ \\
\hline 964.7540 & 964.7559 & 0.99 & 4 & 622.19 & $\mathrm{C1} 183 \mathrm{H} \mathrm{H} 273 \mathrm{~N} 43043$ & $(M+4 N a)+4$ \\
\hline 965.0061 & 965.0066 & 0.48 & 4 & 508.94 & $2183 \mathrm{H} 273 \mathrm{~N} 43043$ & $(\mathrm{M}+4 \mathrm{Na})+4$ \\
\hline 965.2572 & 965.2572 & 0 & 4 & 501.3 & $\mathrm{C} 183 \mathrm{H} 273 \mathrm{~N} 43 \mathrm{O} 43$ & $(\mathrm{M}+4 \mathrm{Na})+4$ \\
\hline 1254.6884 & 1254.6905 & 1.67 & 3 & 663.01 & $\mathrm{C1} 183 \mathrm{H} 273 \mathrm{~N} 43 \mathrm{O} 43$ & $(M+3 H)+3$ \\
\hline 1255.0237 & 1255.0248 & 0.88 & 3 & 1511.46 & $\mathrm{C1} 183 \mathrm{H} 273 \mathrm{~N} 43 \mathrm{O} 43$ & $(M+3 H)+3$ \\
\hline 1255.3583 & 1255.3591 & 0.66 & 3 & 1856.19 & $\mathrm{C} 183 \mathrm{H} 273 \mathrm{~N} 43043$ & $(M+3 H)+3$ \\
\hline 1255.6922 & 1255.6934 & 0.4 & 3 & 1514.3 & $\mathrm{C1} 183 \mathrm{H} 273 \mathrm{~N} 43 \mathrm{O} 43$ & $(M+3 H)+3$ \\
\hline 1256.027 & 1256.0277 & 0.58 & 3 & 967.5 & $\mathrm{C1} 183 \mathrm{H} 273 \mathrm{~N} 43043$ & $(M+3 H)+3$ \\
\hline 1256.3617 & 1256.3619 & 0.2 & 3 & 515.19 & $1183 \mathrm{H} 273 \mathrm{~N} 43043$ & $(M+3 H)+3$ \\
\hline 1256.6958 & 1256.6962 & 0.26 & 3 & 223.84 & $\mathrm{C1} 183 \mathrm{H} 273 \mathrm{~N} 43 \mathrm{O} 43$ & $(M+3 H)+3$ \\
\hline 1257.0286 & 1257,0304 & 1.44 & 3 & 59.65 & C183 273 N43043 & $(M+3 H)+3$ \\
\hline 1276.6671 & 1276.6725 & 4.17 & 3 & 880.63 & $\mathrm{C} 183 \mathrm{H} 273 \mathrm{~N} 43 \mathrm{O} 43$ & $(\mathrm{M}+3 \mathrm{Na})+3$ \\
\hline 1277.0035 & 1277.0068 & 2.55 & 3 & 1244.11 & $\mathrm{C} 183 \mathrm{H} 273 \mathrm{~N} 43 \mathrm{O} 43$ & $(\mathrm{M}+3 \mathrm{Na})+3$ \\
\hline 1277.3387 & 1277.3411 & 1.86 & 3 & 1259.75 & $\mathrm{C} 183 \mathrm{H} 273 \mathrm{~N} 43043$ & $(\mathrm{M}+3 \mathrm{Na})+3$ \\
\hline 1277.6731 & 1277.6754 & 1.76 & 3 & 989.13 & $\mathrm{C1} 183 \mathrm{H} 273 \mathrm{~N} 43 \mathrm{O} 43$ & $(\mathrm{M}+3 \mathrm{Na})+3$ \\
\hline 1278.007 & 1278.0096 & 2.04 & 3 & 632.61 & $\mathrm{C1} 183 \mathrm{H} 273 \mathrm{~N} 43 \mathrm{O} 43$ & $(\mathrm{M}+3 \mathrm{Na})+3$ \\
\hline 1278.342 & 1278.3439 & 1.46 & 3 & 344.81 & $\mathrm{C1} 183 \mathrm{H} 273 \mathrm{~N} 43043$ & $(M+3 \mathrm{Ma})+3$ \\
\hline 1278.6771 & 1278.6781 & 0.82 & 3 & 134.77 & $\mathrm{C1} 183 \mathrm{H} 273 \mathrm{~N} 43043$ & $(\mathrm{M}+3 \mathrm{Na})+3$ \\
\hline 1882.0254 & 1882.0336 & 4.35 & 2 & 71.15 & $\mathrm{C1} 183 \mathrm{H} 273 \mathrm{~N} 43 \mathrm{O} 43$ & $(M+2 \mathrm{H})+2$ \\
\hline 1882.525 & 1882.5351 & 5.35 & 2 & 90.01 & $\mathrm{C1} 183 \mathrm{H} 273 \mathrm{~N} 43 \mathrm{O} 43$ & $(M+2 H)+2$ \\
\hline $1883.02 \approx$ & 1883.0365 & 4.1 & 2 & 64.9 & $\mathrm{C1} 183 \mathrm{H} 273 \mathrm{~N} 43043$ & $(M+2 H)+2$ \\
\hline
\end{tabular}

-- End Of Report -- 